

\section{DISCLAIMER}

This report was prepared as an account of work sponsored by an agency of the United States Government. Neither the United States Government nor any agency Thereof, nor any of their employees, makes any warranty, express or implied, or assumes any legal liability or responsibility for the accuracy, completeness, or usefulness of any information, apparatus, product, or process disclosed, or represents that its use would not infringe privately owned rights. Reference herein to any specific commercial product, process, or service by trade name, trademark, manufacturer, or otherwise does not necessarily constitute or imply its endorsement, recommendation, or favoring by the United States Government or any agency thereof. The views and opinions of authors expressed herein do not necessarily state or reflect those of the United States Government or any agency thereof. 


\section{DISCLAIMER}

Portions of this document may be illegible in electronic image products. Images are produced from the best available original document. 
UCRL-9 183

UC-34 Physics and Mathematics

T ID-4500 (15th Ed.)

\section{UNIVERSIT Y OF CALIFORNIA. \\ Lawrence Radiation Laboratory \\ Berkeley, California}

Contract No. W-7405-eng-48

A FURTHER STUDY OF ANTIPROTON INTERACTIONS

AND THE ANNIHILATION PROCESS

Rein Silberberg

(Thesis)

Apri1 $11, \ldots 1960^{\circ}$ 
Printed in USf pice \$1.75. Available from the office of Technical Services U. S. Department of Commerce Washington 25, D.C. 
A FURTHER STUDY OF ANTIPROTON INTERACTIONS AND THE ANNIHILATION PROCESS

\section{Contents}

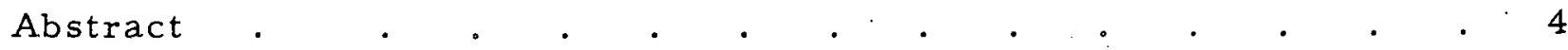

I. Introduction . . . . . . . . . . . . 5

II. The Propane Bubble Chamber Experiment . . . . . . . . . . $\quad$. 7

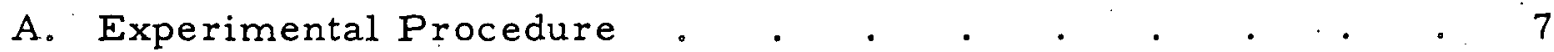

1. The Antiproton Beam . . . . . . . . . . . . . 7

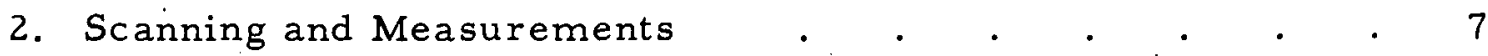

3. The Beam Composition . . . . . . . . . 7

B. Annihilation Cross Sections . . . . . . . . . . 10

C. Charge-Exchange Mean Free Path . . . . . . . 11

D. The Annihilation Process . . . . . . . . . . . 12

1. Annihilation in Carbon Nuclei . . . . . . . . . 12

2. Annihilation with Hydrogen . . . . . . . . . 19

a. Pion Multiplicity . . . . . . . . . . 19

b. Comparison with the Statistical Model a : . . 19

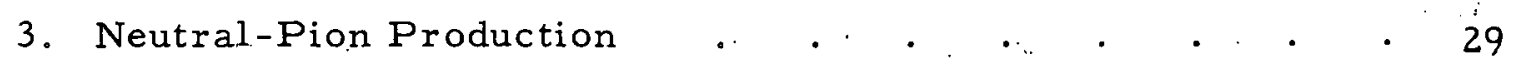

4. Strange-Particle Production . . . . . . . . $\quad .30$

a. Introduction $. \quad . \quad . \quad . \quad . \quad . \quad 30$

b. Determination of Detection Efficiency. . , . . . 30

1. $\mathrm{K}^{0}$. Mesons and $\Lambda$ Hyperons . . . . . . 30

2. Charged $\mathrm{K}$ Mesons . . . . . . . . . 31

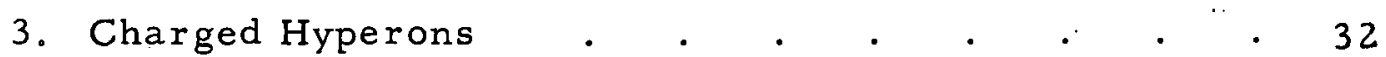

c. $\mathrm{K}^{-}$-Meson Contamination in the Beam . . . . 32

d. The Number of Strange Particles . . . . . . 32

e. Pion Multiplicity in Stars with K Mesons . . . . . 32

f. Momentum Spectra . . . . . . . . . 35

g. Theoretical Analysis of K-Meson Production . - . . . $\quad$.

E. Nuclear Excitation in Carbon . . . . . . . . . . . 41

III. The Emulsion Experiment

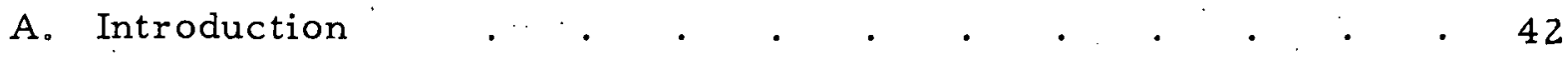

B. Experimental Procedure

1. The Antiproton Beami . . . . . . . . . . . 42 


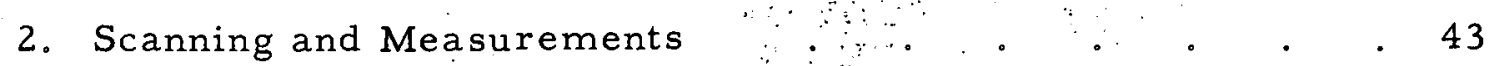

3. Proton Contamination . . . . . . . . . 43

C. Antiproton Cross Sections

1. Antiproton-Proton Elastic Scattering . . . . . . 43

2. Annihilation Cross Sections with Complex Nuclei . . . 44

D. Annihilation in Diluted Emulsions

1. Charged-Pion Multiplicity . . . . . . . . . 52

2. The Ratio of $\overline{\mathrm{p}} \mathrm{p} / \overline{\mathrm{p}} \mathrm{n}$ Annihilations . . . . . . 53

3. The Pion Energy Spectrum . . . . . . 53

4. The $\pi^{+} / \pi^{-}$Ratio at Low Pion Energy . . . . . . 53

5. K-Meson Multiplicity . . . . . 。 . . . . 53

E. Nuclear Excitation in Diluted Emulsions

1. General Data . . . . . . . . . . . . 56

2. The Knock-on Process . . . . . . . . . 56

3. The Evaporation Process . . . . . . . . 62

4. Total Nuclear Excitation and Pion Absorption . . . 63

Acknowledgments . . . . . . . . . . . . . . . 65

Appendices

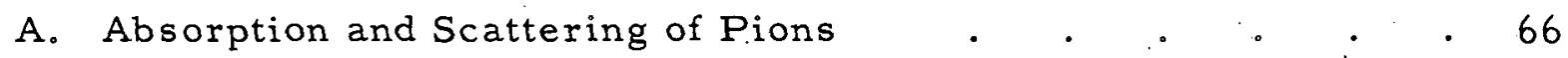

B. Neutral-Pion Absorption . . . . . . . . . 70

Footnotes . . . . . . . . . . . . . . . . . . . 73 


\title{
A FURTHER STUDY OF ANTIPROTON INTERACTIONS
}

\section{AND THE ANNIHILATION PROCESS}

\author{
Rein Silberberg \\ Lawrence Radiation Laboratory \\ University of California \\ Berkeley, California
}

April 11, 1960

\begin{abstract}
The annihilations of $2900 \pm 120 \overline{\mathrm{p}}$ at an average momentum of 1050 $\mathrm{Mev} / \mathrm{c}$ have been analysed in the 30-in. LRL propane bubble chamber. The $\overline{\mathrm{p}}$ annihilation cross sections for hydrogen and carbon were found to be $51 \pm$ $10 \mathrm{mb}$ and $368 \pm 60 \mathrm{mb}$, respectively. The $\overline{\mathrm{p}}$ charge-exchange differential cross section peaks forward strongly. The $\mathrm{K}$-meson multiplicity, $\left\langle\mathrm{N}_{\mathrm{K}} \overline{\mathrm{K}}\right\rangle=$ $8( \pm 1) \%$ per star, is considerably higher than at lower antiproton energies. The primary pion multiplicity in stars with $\mathrm{K}$ mesons is $2.4 \pm 0.5$. The averagc pion multiplicity in stars with no strange particles is $5.0 \pm 0.2$ for both hydrogen and carbon. The width of the pion-multiplicity distribution function has been found to:be in agreement with that predicted by the Fermi statistical model with an increased interaction volume.

In addition, interactions of about 853 antiprotons have been analysed in emulsions: Out of these, 253 annihilated in flight in $3 \times g$ elatin emulsions, and their primary as well as secondary annihilation products were analysed. The $\bar{p}-H$ elastic-scattering cross section for an energy interval from 20 to $230 \mathrm{Mev}$, with an average $\overline{\mathrm{p}}$ energy of $150 \mathrm{Mev}$, is $56 \pm 8 \mathrm{mb}$. The average annihilation cross section is $1720 \pm 175 \mathrm{mb}$ with $\mathrm{AgBr}$, and $546 \pm 105 \mathrm{mb}$ for the light elements in emulsions, excluding hydrogen. The average number of charged pions observed in annihilations in flight in 3xgelatin emulsions is $\left\langle N_{\pi}\right\rangle=2.31 \pm 0.16$. The $\mathrm{K}$-meson multiplicity for annihilations in flight, at an average energy of $150 \mathrm{Mev}$, is again found to be $3.5( \pm 1.5) \%$ per star. The energy in cascade nucleons and nuclear evaporation in 3xgelatin emulsions for annihilations in flight was found to be $442 \pm 40 \mathrm{Mev}$ per star, which corresponds to the absorption of $1.1 \pm 0.20$ pions per annihilation. The angular distribution of cascade protons is strongly dependent on the energy of the protons.
\end{abstract}




\section{INTRODUCTION}

The concept of antiparticles was introduced by Dirac by subjecting the wave equation of quantum mechanics to the requirements of special relativity.

The confirmation of this theory by the discovery of the positron led to attempts to search for antinucleons in cosmic radiation or to produce them artificially. The first successful investigation was carried out in 1955 by Chamberlain, Segrè, Wiegand, and Ypsilantis, ${ }^{l}$ who verified the predictions of the Dirac theory concerning the charge and mass of the antiproton. The annihilation of antiproton-nucleon pairs was confirmed soon thereafter. ${ }^{2} \mathrm{~A}$ more extensive study of antiproton interaction cross sections and the antiproton annihilation process with counters, emulsions, and bubble chambers followed these first exploratory experiments.

The problems of particular interest to theoretical physicists have been the large $\bar{p}$ scattering and annihilation cross sections, the angular correlations between pairs of charged pions, and the large pion multiplicity in the annihilalion process.

The large $\bar{p}$ scattering and annihilation cross sections have been explained by Ball, Chew, and Fulco. ${ }^{3-5}$ Their theory is described in greater detail in Section III-C.

The elastic scattering of antiprotons in complex nuclei has been describ $=$ ed by Glassgold. ${ }^{6} \mathrm{He}$ used an optical-model potential with a large imaginary part to take into account the large annihilation cross section.

Angular correlations between pions of like charges as wêll as between pions of unlike charges have been found.' These results have been discussed by Goldhaber et al. 7

Various attempts have been made to explain the large pion multiplicity. Attempts to apply Fermi's statistical theory ${ }^{8-11}$ to anitiproton annihilations lead to an interaction volume about ten times larger than that estimated from the pion Compton wavelength; and to too high a $\mathrm{K} \overline{\mathrm{K}}$ meson abundance.

In an attempt to avoid the large reaction volume, a number of different proposals have been made. Koba and Takeda have considered the annihilation process as a two-step process. ${ }^{12}$ At first the cores of the nucleon and antinucleon annihilate with the emission of 2.2 pions on the average. As a second step, the pion cloud breaks. up. giving rise to $\sim 2.6$ pions. (Since the period of oscillation of pions in the cloud is longer than the time of core annihilation, 
the pion cloud will not interact appreciably with the core during the core annihilation). This model gives $\left\langle N_{\pi}\right\rangle=4.8$ if $\mathrm{K} \overline{\mathrm{K}}$ pair production is neglected. However, also this model leads to too high a $\mathrm{K} \overline{\mathrm{K}}$ abundance $(\sim 15 \%){ }^{13}$

Another possibility is to consider the effects of pion-pion interactions in the final state. This pion-pion interaction would raise the average pion multiplicity as obtained in the Fermi model. This approach was discussed by Pomeranchuk, ${ }^{14}$ Eberle, ${ }^{15}$ Gotô, $^{16}$ and more recently by Cerulus. ${ }^{17}$

A recent summary of various attempts to predict a high pion multiplicity is given by Frautschi. ${ }^{13}$ A summary of both experimental and theoretical research on antinucleons in its various phases is given by Segre. 18

The experiment described here consists of two parts; (a) a propane bubble chamber exposure of antiprotons with a momentum of $1050 \mathrm{Mev} / \mathrm{c}$, and (b) an exposure of diluted emulsions to an antiproton beam with a momentum of $730 \mathrm{Mev} / \mathrm{c}$.

The present work leans strongly on the Antiproton Collaboration Experiment (here referred to as ACE), ${ }^{11}$ and on our publication of the AntiprotonNucleon Annihilation Process II (here referred to as II). ${ }^{19}$ Definitions for any expressions that are used but not explained in this paper can be found in $A C E$ or II.

Our experimental results for $\bar{p}-p$ elastic scattering further confirm the theory of Ball, Chew, and Fulco. ${ }^{3-5}$ The experimental cross sections and the $\bar{p}-p$ differential cross section are in good agreement with their predictions.

We have found that the $\bar{p}-p$ charge-exchange differential cross section at $475 \mathrm{Mev}$ peaks strongly in the forward direction.

We have observed a considerably higher $\mathrm{K}$-meson multiplicity in our experiment with high-energy antiprotons, $[8( \pm 1) \%]$. The results of lowenergy experiments give $4( \pm 1) \%$. $11,20,21$

The pion multiplicity, on the other hand, has not increased noticeably with the incoming antiproton energy. The pion multiplicity has also been found to be the same for annihilations with free and bound nucleons.

In Sections II-D-2 and II-D-4 we investigate the predictions of the Fermi statistical model and the Koba-Takeda model regarding the increase in the $\pi$ - and $\mathrm{K}$-meson multiplicity with increasing annihilation energy.

In Section II-D-2 we also discuss the effect of the width of the pion- multiplicity distribution and conclude that the distribution function of the correct model must not differ much from that derived from the Fermi statistical model with a large interaction volume. 


\section{THE PROPANE BUBBLE CHAMBER EXPERIMENT}

\section{A. Experimental Procedure}

$\therefore 1$. The Antiproton Beam

A study of antiproton interactions with hydrogen and carbon nuclei was carried out using the Berkeley 30-in. propane bubble chamber as a detector. The momentum of the antiprotons was $1050 \mathrm{Mev} / \mathrm{c}$.

To reduce the large background of particles other than antiprotons, electrostatic velocity selectors were used inthecconstruction of the beam setup. The beam design and setup was carried out by Eberhard, Good, and. Ticho for a $\mathrm{K}^{-}$experiment that preceded the $\overline{\mathrm{p}}$ experiment. ${ }^{22}$ Appropriate tuning of the velocity spectrometers permitted us to adjust the system to transmit antiprotons rather than $\mathrm{K}^{-}$mesons.

2. Scanning and Measurements

In the scanning of the film, all interactions were recorded and classified according to their interaction products. All pictures were scanned twice. In both scans, upon locating an interaction each scanner gave his interpretation of the event and attempted to identify all prongs and decay of the neutral secondaries. The identification was based on ionization, momentum, and dip angle. In some cases identification of the particle was facilitated by inter-

factions, decay, or the particle's coming to rest. The number of usable pictures in the film was 20141.

Events of special interest, such as production of strange particles, $\overline{\mathrm{p}}-\mathrm{p}$ scatters, and hydrogen-like antiproton-annihilation events were further analysed by using the Frankenstein and IBM computing facilities to measure the momenta, ranges, angles, coplanarity, and $Q$ values for decays.

Because of the high beam momentum, the main difficulty in determining the number of antiproton annihilation events is the pion contamination; a number of stars with, two or less pions emitted are actually pion-initiated stars, and identification on the basis of the ionization of the incoming track is possible only when an identified track is present in the same section of the chamber in the same picture.

3. The Beam Composition

The following criteria were used to determine the actual $\bar{p}$ path length in the experiment:

(a) the projected angles of the $\bar{p}$ entering the chamber, 
(b) elimination of the background $\pi$ and $\mu$ mesons by using energetic $\delta$ rays formed along the track as identifying criteria,

(c) introducing a geometrical cutoff by using only the central volume of the chamber,

(d) applying a momentum cutoff to the incoming tracks.

The number of $\pi$-stars was evaluated by recording the number of $\delta$ rays with a diameter of $1.5 \mathrm{~cm}$ or more on interacting tracks. A diameter of 1.5 $\mathrm{cm}$ corresponds to a momentum of $3^{-} \mathrm{Mev} / \mathrm{c}$ for our particular magnetic field strength. At our beam momentum, antiprotons do not produce such energetic $\delta$ rays. According to our experimental determination, based on a bubble chamber exposure to a pion beam of similar momentum, one such $\delta$ ray is produced. per $1.25 \pm 0.05$ meters $(\mathrm{m})$ of pion track. The number of pion stars is now directly obtained from the number of $\delta$ rays on the interacting track length.

Most tracks passed through the bubble chamber without visible interactions and could not be individually identified.

In $15 \%$ of the pictures, which were chosen uniformly from all rolls of the bubble chamber film so as to eliminate the results of possible small fluctuations in the beam composition during the exposure, all the tracks satisfying our geometrical and momentum criteria were recorded for a track-length estimate, and the fraction of $\mu^{-}$and $\pi^{-}$mesons in the beam was determined by the $\delta$ ray method. The estimated beam composition is given in Table $\mathrm{I}$. The total estimated number of antiproton tracks satisfying the geometric and momentum-cutoff criteria is $10100 \pm 1700$.

Three methods were used to obtain information on the fraction of $\pi^{-}$ stars in the various subgroups of stars: (a) identification by ionization or $\delta$ rays. (b) partial analysis of $a \pi^{-}$-beam experiment in the propane bubble chamber at a similar momentum, (c) a study of the stars made by particles whose projected angle was off by more than $5 \mathrm{deg}$ from the average beam direction. Only $3.8( \pm 0.4) \%$ of the $\bar{p}$ stars were beyond this cutoff, while $32( \pm 5) \%$ of the $\pi^{-}$stars were beyond. The results on $\pi^{-}$star identification were similar for all three of the methods, and their average was used to evaluate the number of $\bar{p}$ stars in each subgroup. 
The beam composition at the front side of the observable region in the bubble chamber, for particles with a momentum greater than $600 \mathrm{Mev} / \mathrm{c}$ and a projected angle less than or equal to $5 \mathrm{deg}$ from the average antiproton direction.

$\frac{\text { Particles }}{\overline{\mathrm{p}}}$
$\mu^{-}$and $\mathrm{e}^{-}$
$\pi^{-}$

$\%$ in beam

$38.5 \neq 7$

$49.5 \pm 7$

$\mathrm{I} 2.0 \pm 2$ 


\section{B. Annihilation Cross Sections}

Of the $10100 \pm 1700$ antiprotons which contributed to our antiproton path length of $3385 \pm 550 \mathrm{~m}, 90 \pm 30$ underwent charge exchanges and $2900 \pm 120$ annihilations.

The chief sources of the large errors in the above quoted numbers are the difficulty of recognizing the noninteracting tracks individually, and the difficulty in distinguishing pion stars from small $\bar{p}$ stars. The resolution of these problems has already been discussed in Section II-A.

Geometrical and momentum cutoffs have been applied in the calculation of the above quantities. Events were accepted unly within a contral volume of 41 by 26 by $8 \mathrm{~cm}$, the projected angle of the track had to be within 5 deg of the beam direction, and the curvature of the track had to be less than that corresponding to a momentum of $600 \mathrm{Mev} / \mathrm{c}$. The momentum of the beam was rather well-defined and could not be degraded much by passing through the bubble chamber window. The apparent wider spread is due to multiple scattering of the antiprotons in the bubble chamber and errors in measurement of the curvature, which was done by using templets.

The annihilations can be further classified as those occuring in a hydrögen or carbon nucleus. In most annihilations in carbon, nucleons are emitted because of absorption and (or) scattering of pions produced in the primary process: There is frequently also an imbalance in the sum of the pion charges because of pion absorption or $\bar{p}$ annihilation with a neutron. A hydrogen star, on the other hand, must have (a) balance of charge and (h) no nucleons of hyperons can be emitted. The method used to eliminate annihilations in carbon that simulate hydrogen stars by fulfilling conditions (a) and (b) is described below.

Simulated hydrogen events can be produced by $\bar{p}$ annihilation on a neutron followed by absorption of a $\pi^{-}$and the production of knock-on neutrons or evaporation neutrons. They can also be produced by $\bar{p}$ annihilations on bound protons in which no visible knock-on or evaporation protons are emitted. This may be due to (a) an annihilation in which the pions produced in the annihilations are emitted without energy loss in the parent nucleus, or (b) emission of neutrons, protons, or nuclear fragments with an energy lower than that necessary to produce visible recoils from the excited parent nucleus. Information on the number of simulated hydrogen stars can be found from annihilations on neutrons, which can be identified by the excess of negative charge. 
As the carbon nucleus consists of an equal number of protons and neutrons, the number of "fake" free proton-like annihilation stars must be of the order of the number of "fake" free neutron-like annihilation stars (assuming the $\bar{p}-p$ and $\bar{p}-n$ annihilation cross sections to be approximately equal). The pion multiplicity distribution of the "fake" free-proton-like annihilation stars was obtained from the number of stars that have pion charge balance and differ from the "fake" free-proton-like stars only through the emission of protons, multiplied by the interpolated ratio of the "fake" free neutron-like stars (i.e., with $\mathrm{N}_{\pi^{-}}=\mathrm{N}_{\pi^{+}}+1$ ) to those that differ from them only through the emission of protons, at a definite pion multiplicity. Actually the number of "fake" $\overline{\mathrm{p}}-\mathrm{n}$ stars exceeds slightly the "fake" $\overline{\mathrm{p}}-\mathrm{p}$ stars, due to $\pi^{-}$interactions leäding preferentially to neutron emission.

This correction eliminated $16 \%$ of the hydrogen-like stars, giving us $780 \pm 60 \overline{\mathrm{p}}-\mathrm{H}$ annihilations and $2120 \pm 100$ annihilations in carbon nuclei. The cross sections thus obtained are $51 \pm 10 \mathrm{mb}$ for $\overrightarrow{\mathrm{p}}-\mathrm{p}$ annihilation and $368 \pm 60$ $\mathrm{mb}$ for $\overline{\mathrm{p}}$ annihilation in carbon at an antiproton average momentum of 1050 $\mathrm{Mev} / \mathrm{c}$.

\section{Charge-Exchange Mean. Free Path}

The antiproton charge-exchange reaction $\bar{p}+p \rightarrow \bar{n}+n$ can be identified in the bubble chamber by the disappearance of $\bar{p}$ and the subsequent annihilation of the antineutron. We observed $24 \overline{\mathrm{p}}$ disappearances followed by observed $\bar{n}$ annihilations. In addition we observed $109 \pm 30 \bar{p}$ disappearances that were not followed by observed $\overline{\mathrm{N}}$ annihilations. ${ }^{23}$ From the 24 identified charge exchanges, it is possible to evaluate the total number of charge exchanges by first correcting for the detection efficiency of those $\bar{n}$ stars where the number of charged pions emitted in the annihilation is one or less. This correction was done by assuming the fraction of such stars to be the same as in the case of $\bar{p}$ annihilation stars.

Next we have to compute the number of antineutrons that escape the chamber from the observed number of $\bar{n}$ annihilations. All antineutron stars observed in our sample were in the forward hemisphere in the center-of-mass system, and the $\bar{p}$ charge-exchange differential cross section peaked sharply in the forward direction. This indicated that the energy of the antineutrons thus formed was comparable to that of the incoming antiprotons, and justified the assumption that their mean free path was of the same magnitude as that of the antiprotons. 
The number of antineutrons escaping the chamber was estimated from (a) the geometry of the bubble chamber, (b) the positions of $\bar{p}$ disappearances, (c) the $\bar{n}$ annihilation mean free path, (d) the number of $\bar{n}$ annihilations in the chamber and (e) the angular distribution of the antineutrons.

The number of antiprotons satisfying our cutoff criteria that undergo charge exchange is $90 \pm 30$. This leads to an antiprton charge-exchange mean free path of $38 \pm 14 \mathrm{~m}$ in propane at our particular antiproton energy.

On the basis of present information about the $\bar{p}$-p charge-exchange cross section, 24-26 we can conclude that most of uur chat ge exchangoe are with hydrogen nuclei. This is further supported by the fact that of the 24 antineutron annihilations observed, none originated from a source that exhibited nuclear excitation.

Figure 1 gives the $\bar{p}$-p charge-exchange angular distribution. 27 The data given in Fig. 1 probably includes several charge exchanges in carbon nuclei, but corresponds mainly to $\bar{p}-\mathrm{H}$ events. A $16-\mathrm{cm}$ cutoff applied to the range of the antineutrons makes our sample unbiased for the interval $1 \geq \cos \left(\theta_{\overline{\mathrm{p}}-\overline{\mathrm{n}}}\right)$ c. ml. $\geq 0.5$. For larger angles, an increasingly larger fraction of the $\bar{n}$ would escape the chamber.

On the basis of all events with an $8-\mathrm{cm}$ cutoff, we can set an upper limit of $20 \%$ for $\vec{p}$ charge-exchange events with a scattering angle of 60 deg or more in the center-of-mass system. ${ }^{20}$ Figure 1 shows definitely that the $\bar{p}-p$ chargeexchange cross section peaks strongly forward at an antiproton momentum of $1050 \mathrm{Mev} / \mathrm{c}$.

A forward peaking in the $\bar{p}-p$ charge-exchange differential cross section is predicted by Ball and Fulco ${ }^{5}$ at $750 \mathrm{Mev} / \mathrm{c}$. They predict also a second peak at $180 \mathrm{deg}$ in the c.m. system. However, the present results which show a more pronounced forward peaking cannot be compared directly with their predictions, because the approximation methods used by these authors break down at higher antiproton momenta.

\section{The Annihilation Process}

1. Annihilation in Carbon Nuclei

The annihilation process in the propane bubble chamber is in some respects easier to analyse than that in emulsions. The rragnetic field ( 14 kgauss) used in the 30-in. propane bubble chanber enables one to determine the charges of pions. The average $\gamma$-ray radiation length in propane 


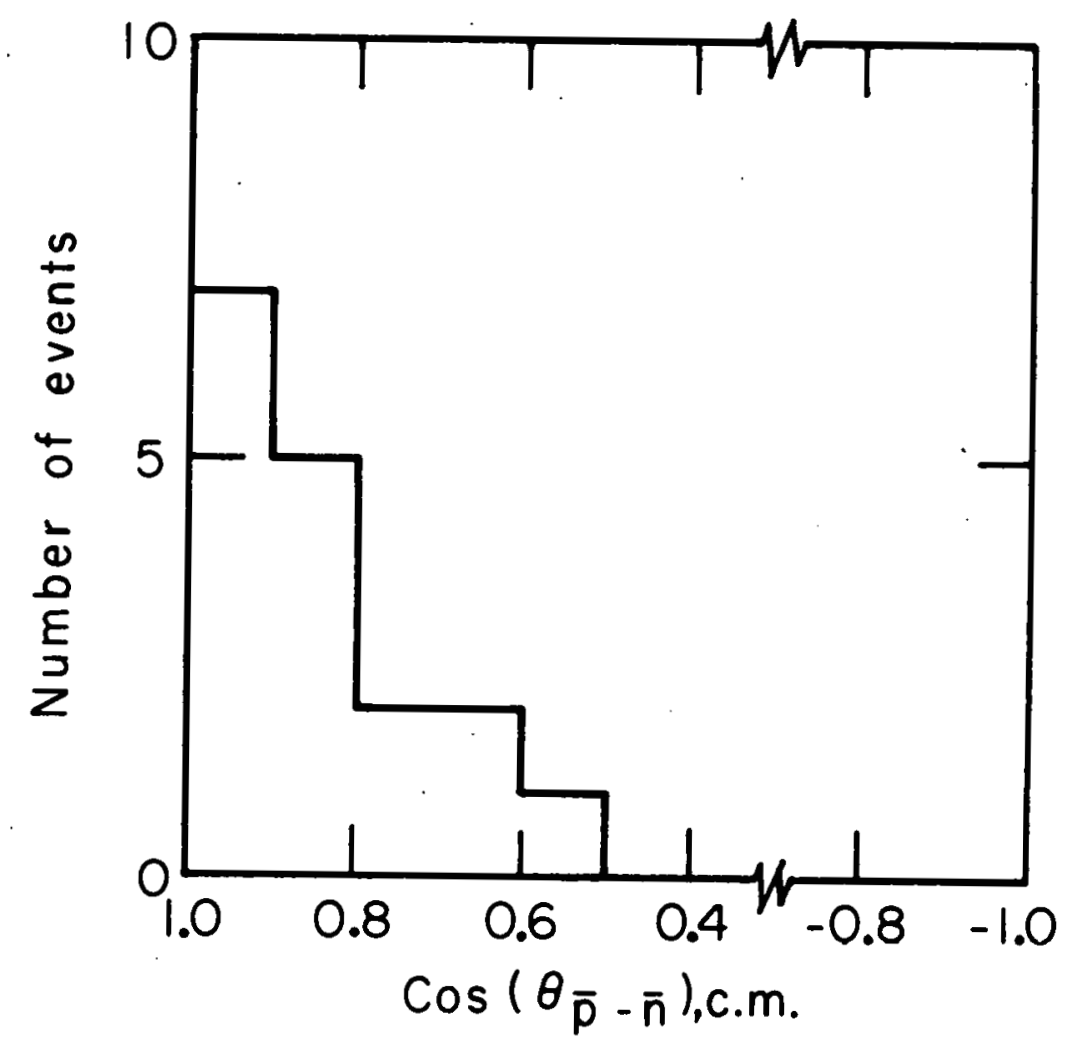

$M U-20004$

Fig. 1. The uncorrected $\overline{\mathrm{p}}-\mathrm{p}$ charge-exchange angular distribution at an antiproton momentum of $1050 \mathrm{Mev} / \mathrm{c}$. The data in this figure probably include some charge exchanges in carbon nuclei. A $16-\mathrm{cm}$ cutoff applied to the range of the antineutrons makes our sample unbiased for the interval $1>\cos (\theta \bar{p}-\bar{n}) \mathrm{c} . \mathrm{m} .>0.5$. For larger angles, an increasingly larger frāction of the antineutrons would escape the chamber. 
permits one to detect about $15 \%$ of the neutral pions through pair production of the decay $\gamma$ rays in the chamber. An attempt was also made to separate the annihilations in hydrogen from those in carbon nuclei.

Without pion absorption, the number of pions of given charge in an annihilation star is restricted by charge conservation. Thus for $\bar{p}-p$ annihilation the number of negatively charged pions equals those positively charged, while in $\bar{p}-n$ annihilation, the number of negative pions exceeds the number of positive pions by one.

Some antiprotons undergo charge-exchange in the carbon nucleus befüie annihilation. We shall assume that the $\bar{p}-p$ charge-exchange cross section is $12 \%$ of its annihilation cross section. 24 Of this $12 \%$ we estimate that about $80 \%$ of the $\bar{n}$ are captured in the parent nucleus, and undergo annihilation with either a proton or a neutron. The resulting $\bar{n}-\mathrm{p}$ annihilation leads to an excess of $\pi^{+}$ over $\pi^{-}$instead.

In this section, $\mathrm{K}$-meson production will be neglected, and stars with strange particles have been left out of the present analysis.

Table II gives the annihilation modes of annihilation stars in carbon. Table III gives the estimated percentage of stars in each charge subgroup, assuming the equality of $\bar{p}-p$ and $\bar{p}-n$ annihilation cross sections, and assuming the pion multiplicity distribution of the Fermi statistical model, modified so as to make the phase space Lorentz-invariant 29 An interaction volume $\Omega=10 \Omega^{0}$ was used, where $\Omega^{0}$ is evaluated from the pion Compton wavelength. On the basis of arguments presented in the following section, we shall use the same pion-multiplicity distribution function as for annihilations at rest.

Strange-particle production has been neglected in these calculations.

In order to compare this Table with our experimental data, it is necessary to correct the initial pion multiplicity for pion absorption in the parent nuclei. Here we consider annihilation events up to $4 \pi^{+}$and $4 \pi^{-}$mesons. The correction is evaluated by use of the equation

$$
F(m, n)=\sum_{i=m}^{4} \sum_{j=n}^{4} U(i, m ; j, n) f(i, j)
$$

where $F(m, n)$ is the fraction of stars having $m \pi^{+}$and $n \pi^{-}$after absorption. Here $f(i, j)$ is the fraction of antiproton stars having $i \pi^{+}$and $j \pi^{-}$before absorption, and $U(i, m ; j, n)$ is the probability that a star with $i \pi^{+}$and $j \pi^{-}$will have $(i-m) \pi^{+}$and $(j-n)$ mesons absorbed. By the use of the binomial theorem, 
Table II

Various possible annihilation modes of annihilation stars in carbon.

Strange-particle production is neglected.

\begin{tabular}{c|ccccc}
\hline$N_{\pi}$ & \multicolumn{5}{|c}{$N_{\pi t}$} \\
\cline { 2 - 5 } & 0 & 1 & 2 & 3 & 4 \\
\hline 0 & $\bar{p}-p, \bar{n}-n$ & $\bar{n}-p$ & & \\
1 & $\therefore \bar{p}-n$ & $\bar{p}-p, \bar{n}-n$ & $\bar{n}-p$ & $\bar{n}-p$ & \\
2 & - & $\bar{p}=n$ & $\bar{p}-p, \bar{n}-n$ & $\bar{p}-p, \bar{n}-n$ & $\bar{n}-p$ \\
3 & & & $\bar{p}-n$ & $\bar{p}-n$ & $\bar{p}-p, \bar{n}-n$ \\
4 & & & & & \\
\hline
\end{tabular}

Table III

Estimated percentage of annihilation stars in carbon in each charge subgroup.

The multiplicity distribution is obtained from the statistical model with Lorentz invariant phase space as evaluated by Desai ${ }^{29}$ for an interaction volume $\Omega=10 \Omega^{0}$. The calculations were made for the primary process (i.e. before pion absorption), neglecting strange particle production. The $\bar{p}-\mathrm{p}$ chargeexchange cross section was assumed to be $12 \%$ of the annihilation cross section, and the $\bar{p}-p$ and $\bar{p}-n$ annihilation cross sections were assumed to be equal.'

\begin{tabular}{l|ccccc}
\hline \multirow{2}{*}{$N_{\pi}$} & \multicolumn{5}{|c}{$\mathrm{N}_{\pi+}+$} \\
\cline { 2 - 5 } & 0 & 1 & 2 & 3 & 4 \\
\hline 0 & 0.9 & 0.3 & & & \\
1 & 5.2 & 18.3 & 1.6 & & \\
2 & & 27.9 & 27.7 & 0.7 & 0.0 \\
3 & & & 13.8 & 3.3 & 0.0 \\
4 & & & & 0.3 & \\
\hline
\end{tabular}


$\mathrm{U}(\mathrm{i}, \mathrm{m} ; \mathrm{j}, \mathrm{n})$ is evaluated from the probability of absorption of one pion. If $\mathrm{p}$ is the probability of absorption per pion, then we have

$$
U(i, m ; j, n)=\frac{i ! j !}{m ! n !(i-m) !(j-n) !^{i+j-m-n}(l-p)}{ }^{m+n}
$$

Table IV shows how a $15 \%$ probability of absorption per pion modifies Table III. The value of $15 \%$ was arrived at by estimating the pion absorption from the nuclear excitation, evaluated in Section II-E. On the basis of arguments presented in Appendix B, we assumed the probability of absorption per pion to be the same for charged and neutral pions. With this absorption probability per pion, the probability for the absorption of more than $2 \pi^{ \pm}$ becomes negligible.

Table $\mathrm{V}$ gives the corresponding experimental pion distribution of $\overline{\mathrm{p}}$ stars in carbon. The agreement with Table IV is good, and minor discrepan cies can be interpreted as being due to uncertainty in $\pi^{+}$, $p$ identification, as discussed below.

The observed pion multiplicities for annihilation stars in carbon are $1.49 \pm 0.05 \pi^{-}$and $1.35 \pm 0.05 \pi^{-1}$ per star. We shall now investigate the significance of the fact that the difference in the observed $\pi^{-}$and $\pi^{+}$multiplicity is $0.14 \pm 0.07$. If we assume the equality of the $\bar{p}-p$ and $\bar{p}-n$ annihilation cross sections and apply the principle of charge conservation, the expected difference in $\pi^{-}$and $\pi^{+}$multiplicity for $\bar{p}$ annihilation in carbon is 0.50 . If the $\bar{p}-p$ annihilation cross section would exceed that of $\bar{p}-n$, the difference in $\pi^{-}$and $\pi^{+}$multiplicities would be smaller. Huweves, a loole at the number of events with an even or odd number of charged pions in Fig. 2 shows that such an assumption is not justified. Taking into account the facts that (a) the $\bar{p}-p$ charge-exchange cross section is about $12 \%$ of the annihilation cross section, and (b) about $15 \%$ of the pions produced in $\bar{p}$ annihilations in carbon are absorbed in the same nucleus, we obtain 0.38 as the expected difference in $\pi^{-}$and $\pi^{+}$multiplicities. A somewhat greater absorption cross section for $\pi^{-}$than for $\pi^{+}$due to the Coulomb effect could not reduce the difference appreciably. We conclude that some protons-particularly those with a high kinetic energy or those with large dip angles were considered to be $\pi^{+}$mesons.

The charged-pion multiplicity distribution for annihilations in carbon nuclei is given in Fig. 2. The data are uncorrected for pion absorption. 
Table IV

Modification of Table III by $15 \%$ pion absorption.

\begin{tabular}{l|ccccc}
\hline \multirow{2}{*}{$\mathrm{N}_{\pi-}$} & \multicolumn{5}{|c}{$\mathrm{N}_{\pi^{+}}$} \\
\cline { 2 - 6 } & 0 & 1 & 2 & 3 & 4 \\
\hline 0 & 2.2 & 3.1 & 0.6 & 0.0 & 0.0 \\
1 & 8.1 & 21.3 & 6.6 & 0.1 & 0.0 \\
2 & 4.2 & 23.1 & 17.8 & 1.0 & 0.0 \\
3 & 0.2 & 2.1 & 6.8 & 1.3 & 0.0 \\
4 & 0.0 & 0.0 & 0.1 & 0.1 & 0.0 \\
\hline \hline
\end{tabular}

Table V

Experimental pion distribution of $\bar{p}$ stars in carbon, in percent of stars. The error includes only the statistical error and that from the subtraction of $\bar{p}-\mathrm{H}$ and $\pi^{-}$stars from the total number of events.

\begin{tabular}{c|ccccc}
\hline$N_{\pi^{-}}$ & \multicolumn{5}{|c}{$\mathrm{N}_{\pi^{+}}$} \\
\cline { 2 - 6 } & 0 & 1 & 2 & 3 & 4 \\
\hline 0 & $1.9 \pm 0.5$ & $4: 1 \pm 0.5$ & $1.9 \pm 0.2$ & $0.6 \pm 0.1$ & $0.1 \pm 0.1$ \\
1 & $7.2 \pm 1.0$ & $19.6 \pm 1.3$ & $12.6 \pm 0.6$ & $2.2 \pm 0.2$ & $0.2 \pm 0.1$ \\
2 & $3.2 \pm 0.3$ & $20.7 \pm 0.7$ & $11.8 \pm 0.6$ & $4 . .1 \pm 0.3$ & $0.4 \pm 0.1$ \\
3 & $0.5 \pm 0.1$ & $2.7 \pm 0.3$ & $3.8 \pm 0.3$ & $1: 3 \pm 0.2$ & $0.1 \pm 0.1$ \\
4 & $0.0 \pm 0.0$ & $0.1 \pm 0.1$ & $0.2 \pm 0.1$ & $0.1 \pm 0.1$ & $0.0 \pm 0.0$ \\
\hline \hline
\end{tabular}




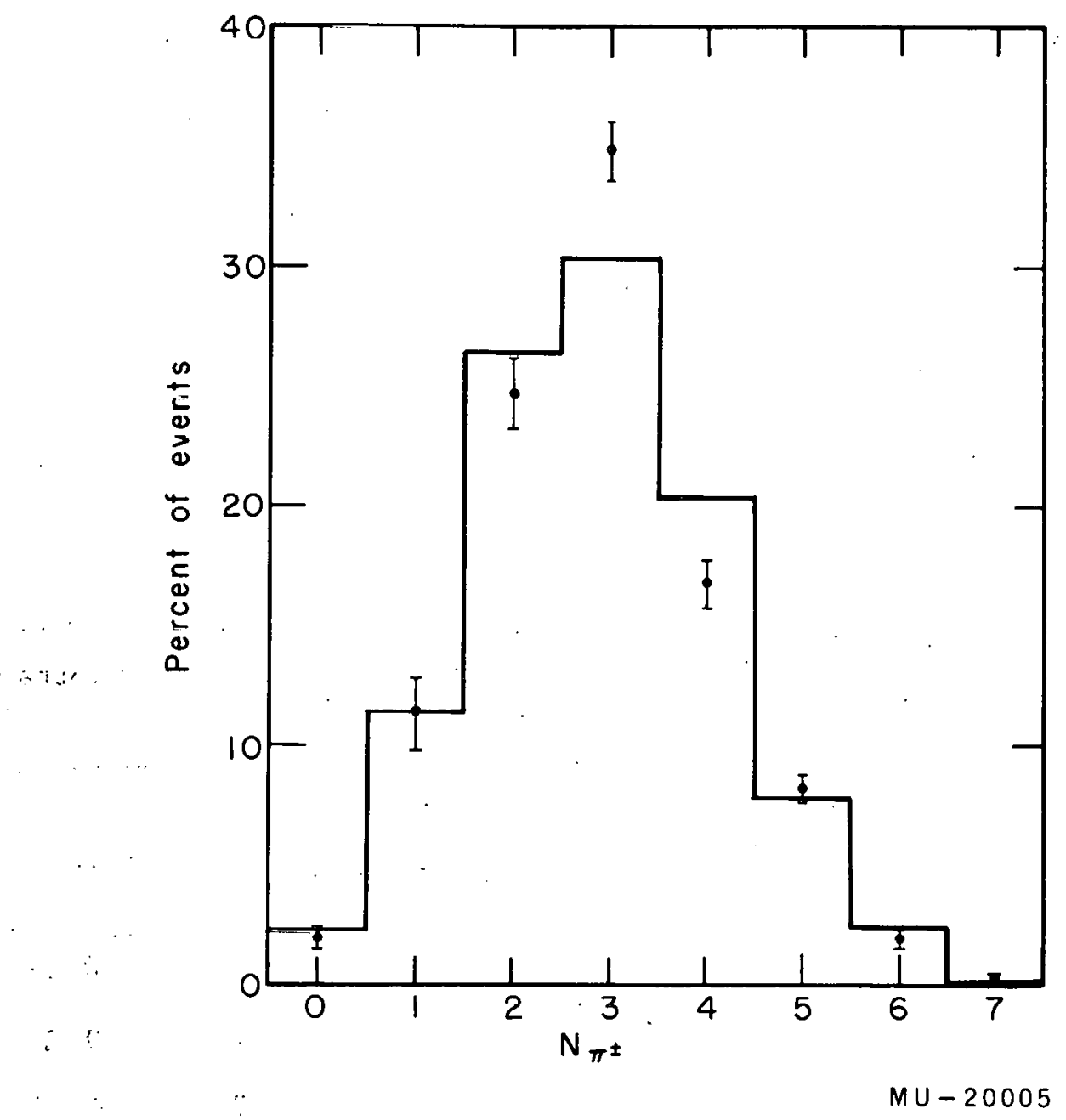

Fig. 2. The charged-pion multiplicity distribution for carbon stars, omitting events with observed strange particles. The solid line is based on calculations with the Fermi statistical model for $\Omega=10 \Omega^{0}$ and modified so as to make the phase space Lorentz-invariant. It was assumed that $15 \%$ of the pions are absorbed. 
The uncertainty in determining the number of stars with two or less pions is. large, since in this group corrections for pion contamination had to be made. The evaluation of errors included also the subtraction of the estimated number of hydrogen annihilation events. No correction has been made for the possibility that some protons were considered to be $\pi^{+}$mesons.

The energies of the pions from $\bar{p}$ annihilations were measured only for the hydrogen-like events. Hence the evaluation of the original pion multiplicity in carbon stars will not be very accurate. The average number of $\pi^{0}$ mesons in annihilation events in carbon and the estimated fraction of pions ahsnrher are given in later sections. The estimated primary pion multiplicity for annihilation events in carbon is $\left\langle N_{\pi}\right\rangle=5.0 \pm 0.2$. In this calculation it was assumed that an excess of $0.24 \pm 0.07$ p per annihilation star were mistaken for $\pi^{+}$.

\section{Annihilation with Hydrogen}

a. Pion multiplicity. The method of determining the number of $\bar{p}-p$ annihilations has been described in Section II-B. The multiplicity of charged mesons per hydrogen annihilation is $\left\langle N_{\pi \pm}\right\rangle=3.3 \pm 0.15$ for stars with no observed $K$ mesons. The error includes (a) the statistical error, (b) the error due to eliminating carbon stars simulating hydrogen events, and (c) the error due to the elimination of $\pi^{-}$stars. The value is very close to that obtained by Agnew et a.l. ${ }^{20}$ and Horwitz et al. ${ }^{25}$ at low energy and indicates that pion multiplicity. does not increase appreciably with the energy of the incoming antiproton.

With the addition of neutral pions, which is evaluated in the following section, the pion multiplicity for stars with no strange particles is $\left\langle\mathrm{N}_{\pi}\right\rangle=5.0 \pm 0.2$, which is the same as in carbon. We conclude that we have not observed any difference in pion mulitplicity for $\bar{p}$ annihilations with free nucleons or with those bound in complex nuclei.

b. Comparison with the statistical model. In Section I we discussed the statistical model and various modifications of it.

Recent calculations by Desai describe the antiproton annihilation in terms of the Fermi statistical model with Lorentz-invariant phase space. ${ }^{23}$ On the basis of his calculations we calculate that for annihilations at rest, an interaction volume $\Omega=10 \Omega^{\circ}$ leads to a pion multiplicity $\left\langle N_{\pi}\right\rangle=5.0$. At an antiproton momentum of $1050 \mathrm{Mev} / \mathrm{c}$, the same interaction volume leads to $\left\langle\mathrm{N}_{\pi}\right\rangle=5.4$, i.e., an increase of 0.4 pions per star. In this evaluation we did not take into account the Lorentz contraction of the interaction volume due to the motion of the antiproton. 
Since we have not found an appreciable increase in the pion multiplicity, it was of interest to investigate whether some modifications of the Fermi model would predict a smaller increase. However, we cannot exclude the possibility that the absence of a noticeable increase in pion multiplicity is due to a statistical fluclualicin.

In the Koba-Takeda model, ${ }^{12}$ the number of pions in the cloud would be expected to be independent of whether the nucleon or antinucleon is at rest or in motion. The increase in multiplicity would be confined to the annihilation of the cores. Assuming an interaction radius $R=(2 / 3) R_{0}$, where $R_{0}$ is the pion Compton wave length, we obtain $\mathrm{N}_{\pi r n r e}=2.1$ for the annihilatior of the cores. At ari antiproton momentum of $1050 \mathrm{Mev} / \mathrm{c}$, we obtain 0.03 as the increase in pion multiplicily for the annihilation of the rores. The increasc in pion multiplicity predicted by the Koba-Takeda model is less hian that cvaluated at the beginning of this section by one order of magnitude.

As a consequence of this, the Koba-Takeda model would predict a considerable increase of the average energy per pion. In the center-of-mass system, this increase arrounts to about $10 \%$ for annihilations taking place at our beam momentum.

In Fig. 3 we show how the pion multiplicity would vary with the antiproton momentum for (a) the Fermi statistical model without taking into account the Lorenz-contraction of the interaction volume due to the motion of the $\bar{p}$, (b) the Fermi statistical model, taking inlu account the Lorentz-contraction, and (c) the Koba-Takeda model.

The reciprocals of the values of Fig. 3 give the values for the expression $\left\langle E_{\pi}\right\rangle / W$, where $W$ is the total annihilation energy. In the Fermi model, the expression $\left\langle E_{\pi}\right\rangle / W$ decreases with increasing annihilation energy, while in the Koba-Takeda model, it remains relatively constant. A comparison of our data on the average pion energies of the hydrogen-like events with four and six $\pi^{ \pm}$with the low-energy data also favors the Koba-Takeda model. ${ }^{20,25}$ The predictions of the Férmi model, however; are off by only about one standard deviation. In our experiment we cannot therefore make a choice between the two models.

Though we have several reasons to criticize the statistical model and its large interaction volume, it provides us with a pion multiplicity distribution function. Comparison of the experimental results with those predicted by this multiplicity-distribution function would show us whether the true distribution-function deviates from that predicted by the statistical model. 
We shall now compare the experimental results with those based on the multiplicity-distribution function of the Fermi statistical model with Lorentzinvariant phase space, as evaluated by Desai. ${ }^{29}$ On the basis of the above argument with the Koba-Takeda model, the utility of evaluating the distribution function for our antiproton energy is questionable. Hence we shall choose the distribution at rest, with $\Omega=10 \Omega^{0}$ for comparison with our experimental data.

For the evaluation of the various annihilation modes we shall use Table XVI in ACE. This assumes charge projections that give equal weight to each isotopic spin state-the usual assumption of any statistical model. This premise has recently been questioned by Pais, who pointed out the importance of actually measuring the individual charge projections in the annihilation stars. 30 In the present work this was not possible and we will continue to use the statistical-model assumptions.

Figures 4 and 5 give the momentum spectra of the hydrogen-like events with $\mathrm{N}_{\pi^{ \pm}}=4$ and 6 respectively in the center-of-mass system, and the corresponding normalized curves for the Fermi statistical model with an interaction volume $\Omega=10 \Omega^{0}$. The agreement between the experimental data and the Fermi statistical model is good.

Figure 6 gives the multiplicity distribution of charged pions in $\bar{p}-\mathrm{H}$ annihilation events for stars with no observed strange particles. ${ }^{31}$. The curve corresponding to the Fermi model with Lorentz-invariant phase space is drawn in. The agreement with the experimental data is again good.

We shall now compare (a) the average number of neutral pions and (b) the average total energy per charged pion for events having a given number of charged pions with the values predicted by the statistical model. In order to obtain an appreciation of the sensitivity of this test for the width of the pionmultiplicity distribution function, we compute the quantities $(a)$ and (b) for the following extreme multiplicity-distribution functions: (1) a $\delta$ function at $N_{\pi}=5$, viz. $P_{i}=\delta_{i 5}$ and (2) a flat "uniform" distribution $P_{i}=0.2$ for $i=3$ to 7 . Here $P_{i}$ is the fraction of annihilation events having $i$ pions. Both of these distributions give $\left\langle N_{\pi}\right\rangle=5.0$. These comparisons are summarized in Table VI. We see that the agreement with the Fermi multiplicity distribution function is good, and the extreme models can definitely be rejected. Unfortunately, because of $\pi^{-}$- star contamination, the events with two $\pi^{ \pm}$were not usable for comparison $b$. 


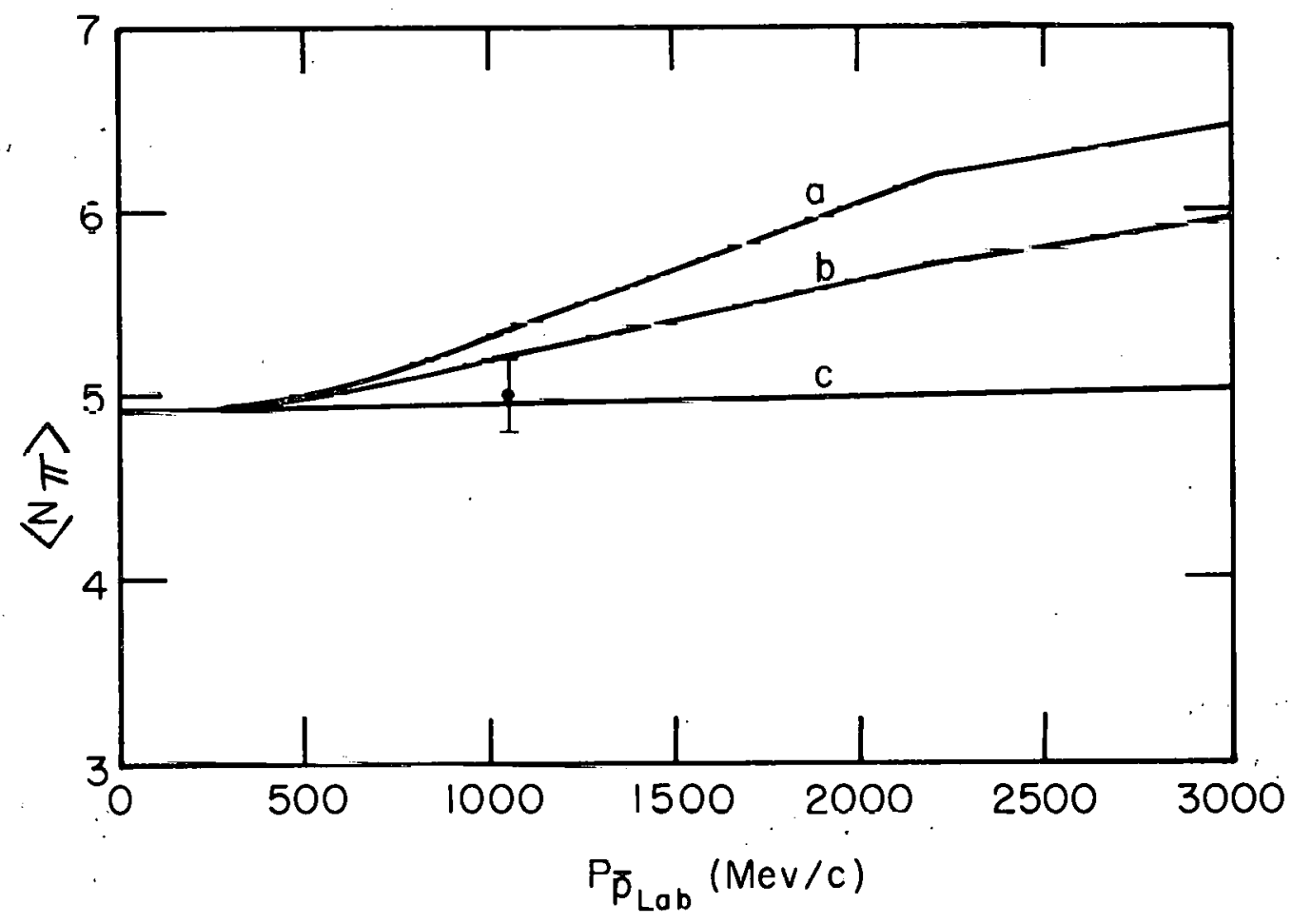

$M U-20006$

Fig. 3. The dependence of the pion multiplicity in $\bar{p}-p$ annihilation on the antiproton momentum. Curves (a) and (b) have been evaluated for the Fermi statistical model. Curve (a) does not take into account the Lorentz contraction of the interaction volume due to the motion of the antiproton, while curve (b) takes it into account. Curve (c) has been evaluated for the Koba-Takeda model. The curves have been evaluated for the case of Lorentz-invariant phase space. The curves have been normalized to give $\left\langle N_{\pi}\right\rangle=4.9$ for annihilations at rest, which is in agreement with the experimental data of Agnew et al.20 and Horwitz et al. 25 on $\bar{p}-p$ annihilations. 


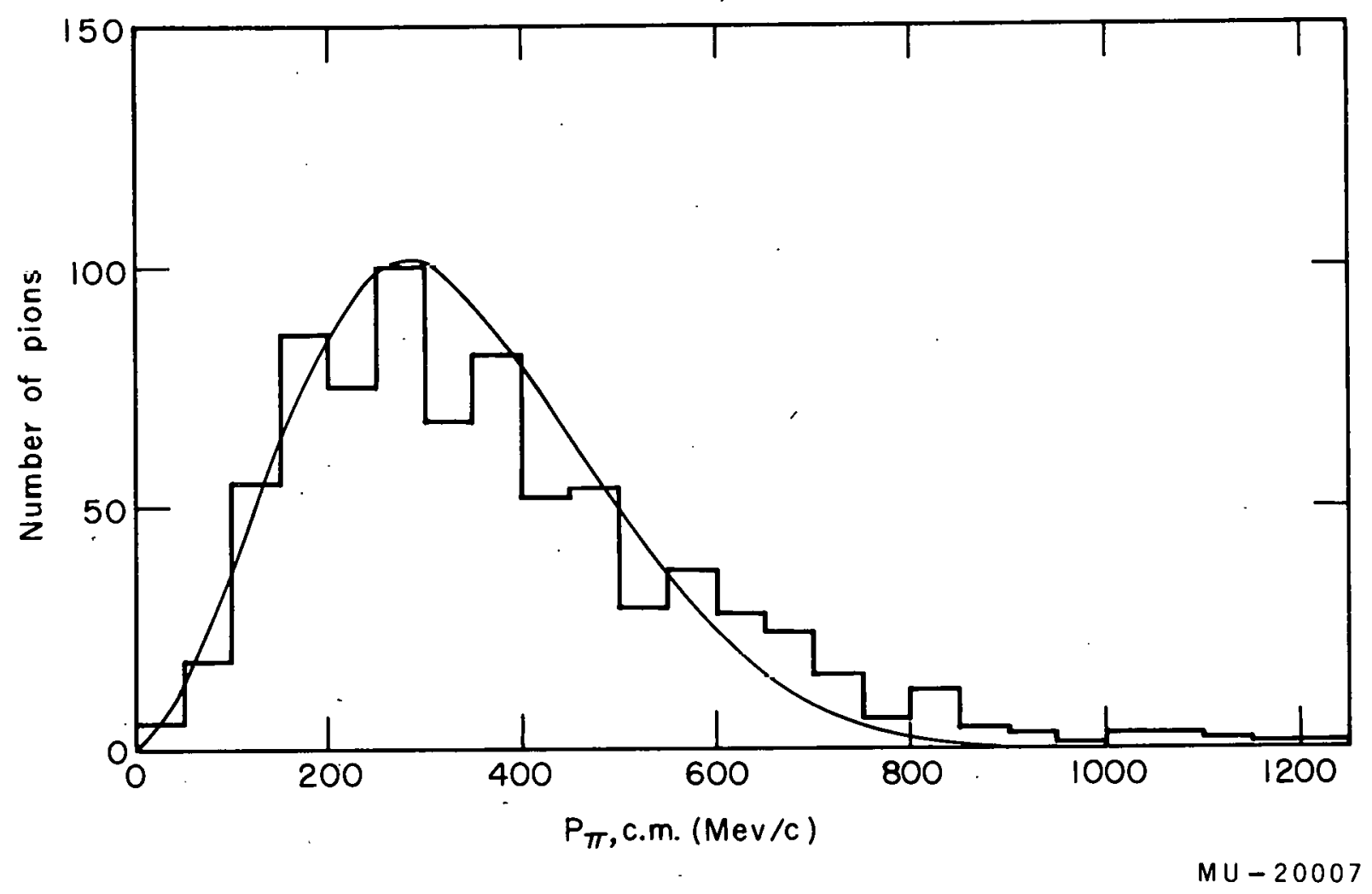

Fig. 4. The pion momentum distribution for hydrogen-like annihilation events with four $\pi^{ \pm}$. The curve for the Fermi model with $\Omega=10 \Omega^{0}$ is also given. 


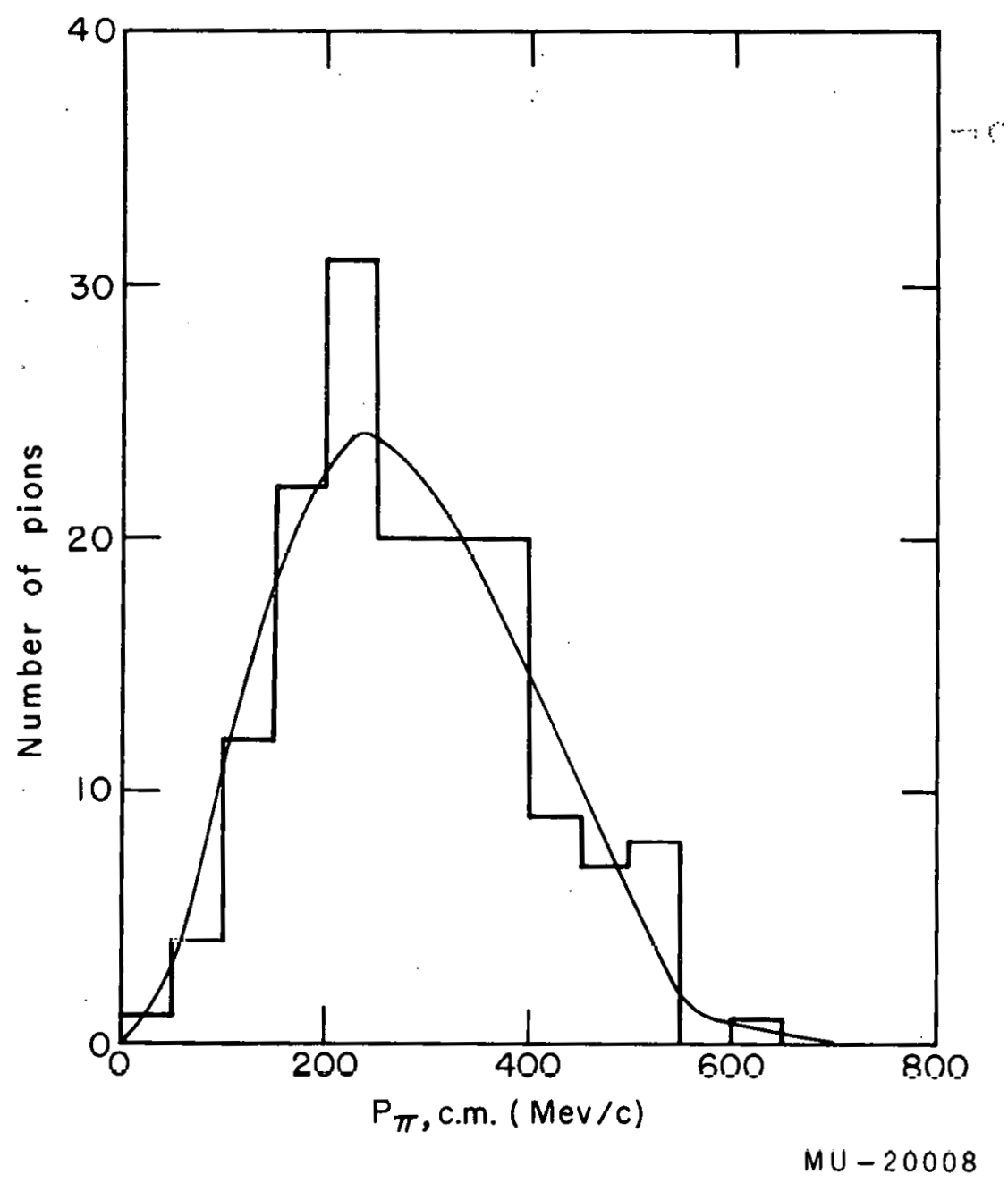

Fig. 5. The pion momentum distribution for hydrogen-like annihilation events with six $\pi^{ \pm}$. The curve for the Fermi model with $\Omega=10 \Omega^{0}$.is also given. 


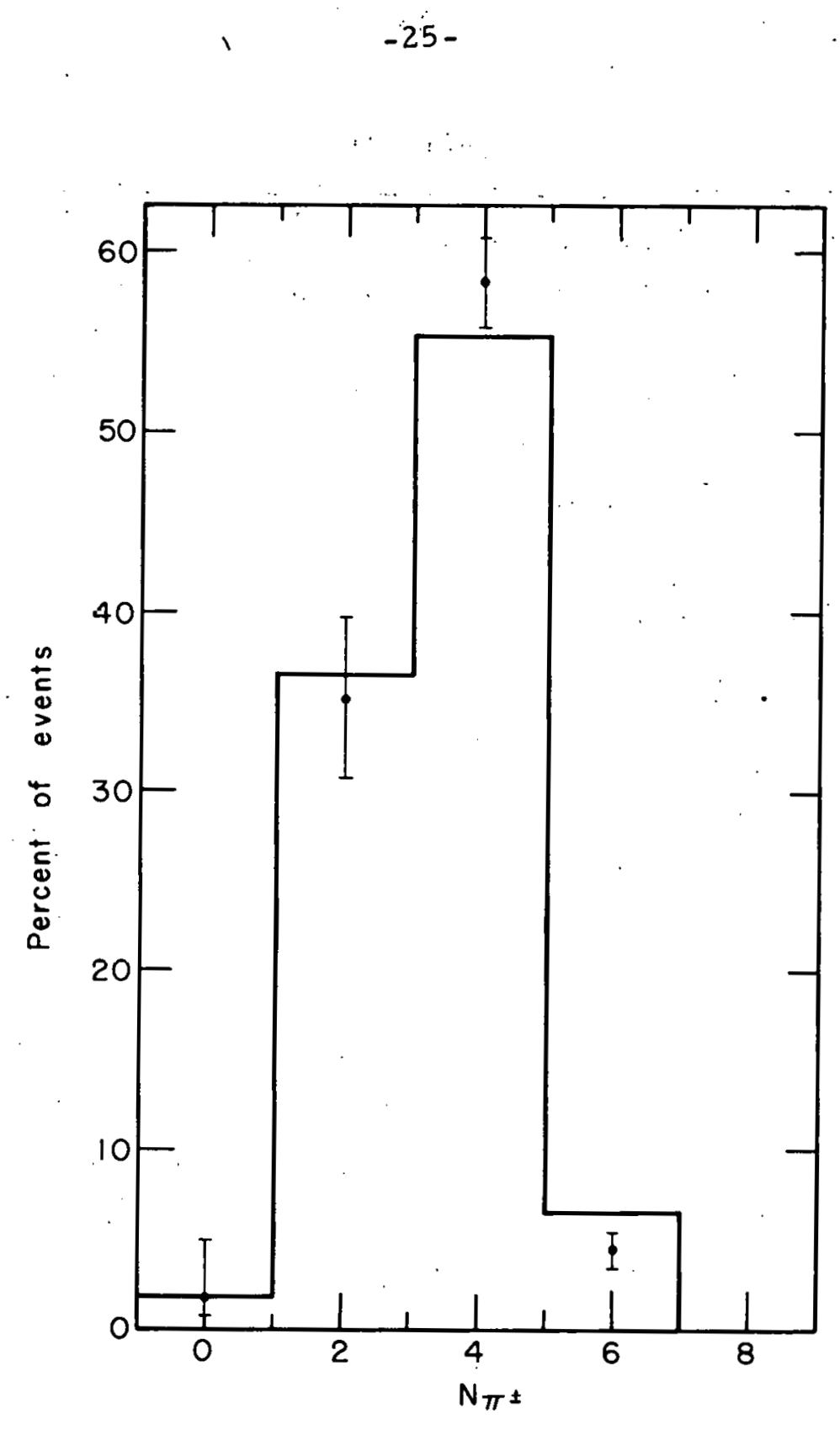

UCRL-9 183

$M U-20009$

Fig. 6. The charged-pion multiplicity distribution for hydrogen stars, omitting events with strange particles. The solid line is based on the Fermi statistical model with $\Omega=10 \Omega^{0}$ and modified to make the phase space Lorentz-invariant. 
Table VI

Average number of neutral pions and average total energy per charged pion for $\overline{\mathrm{p}}-\mathrm{H}$ annihilation events having a given number of charged pions. The experimental data are compared with the predictions of (a) the Fermi statistical model with Lorentz-invariant phase space and an interaction volume $\Omega=10 \Omega^{0}$, (b) a "uniform" distribution with $P_{i}=0.2$ for $i=3$ to 7 , and (c) a " $\delta$-function" distribution with $P_{i}=\delta_{i 5}$. Here $P_{i}$ is the fraction of annihilation events having i pions.

\begin{tabular}{|c|c|c|c|c|c|}
\hline \multicolumn{3}{|c|}{ Experimental data } & \multicolumn{3}{|c|}{ Statlstical model } \\
\hline $\mathrm{N}_{\pi} \pm$ & $\left\langle\mathrm{N}_{\pi \mathrm{r}} \mathrm{O}\right\rangle$ & $\left\langle E_{\pi} \pm\right\rangle$ & $\left\langle\mathrm{N}_{\pi \pm}\right\rangle$ & $\left\langle N N_{0} 0\right\rangle$ & $\left\langle E_{\pi \pm}\right\rangle$ \\
\hline 0 & - & - & 0 & 4.25 & - \\
\hline 2 & $.2 .5 \pm 0.5$ & - & 2 & 2.61 & 532 \\
\hline 4 & $1.1 \pm 0: 1$ & $461 \pm 9$ & 4 & 1.21 & 461 \\
\hline \multirow[t]{2}{*}{6} & $0.8 \pm 0.4$ & $367 \pm 15$ & 6 & 0.32 & 374 \\
\hline & \multicolumn{2}{|c|}{ Uniform model } & \multicolumn{3}{|c|}{$\delta$-function model } \\
\hline $\mathrm{N}_{\pi}^{ \pm}$ & $\left\langle N_{\pi} 0\right\rangle$ & $\left\langle\mathrm{E}_{\pi} \pm\right\rangle$ & $N_{\pi} \pm$ & $\left\langle\mathrm{N}_{\pi} 0\right\rangle$ & $\left\langle E_{\pi} \pm\right\rangle$ \\
\hline 0 & 3.53 & - & 0 & 5.00 & - \\
\hline 2 & 2.01 & 628 & 2 & 3.00 & 470 \\
\hline 4 & 1.50 & 438 & 4 & 1.00 & 470 \\
\hline 6 & 0.69 & 353 & 6 & - & - \\
\hline
\end{tabular}




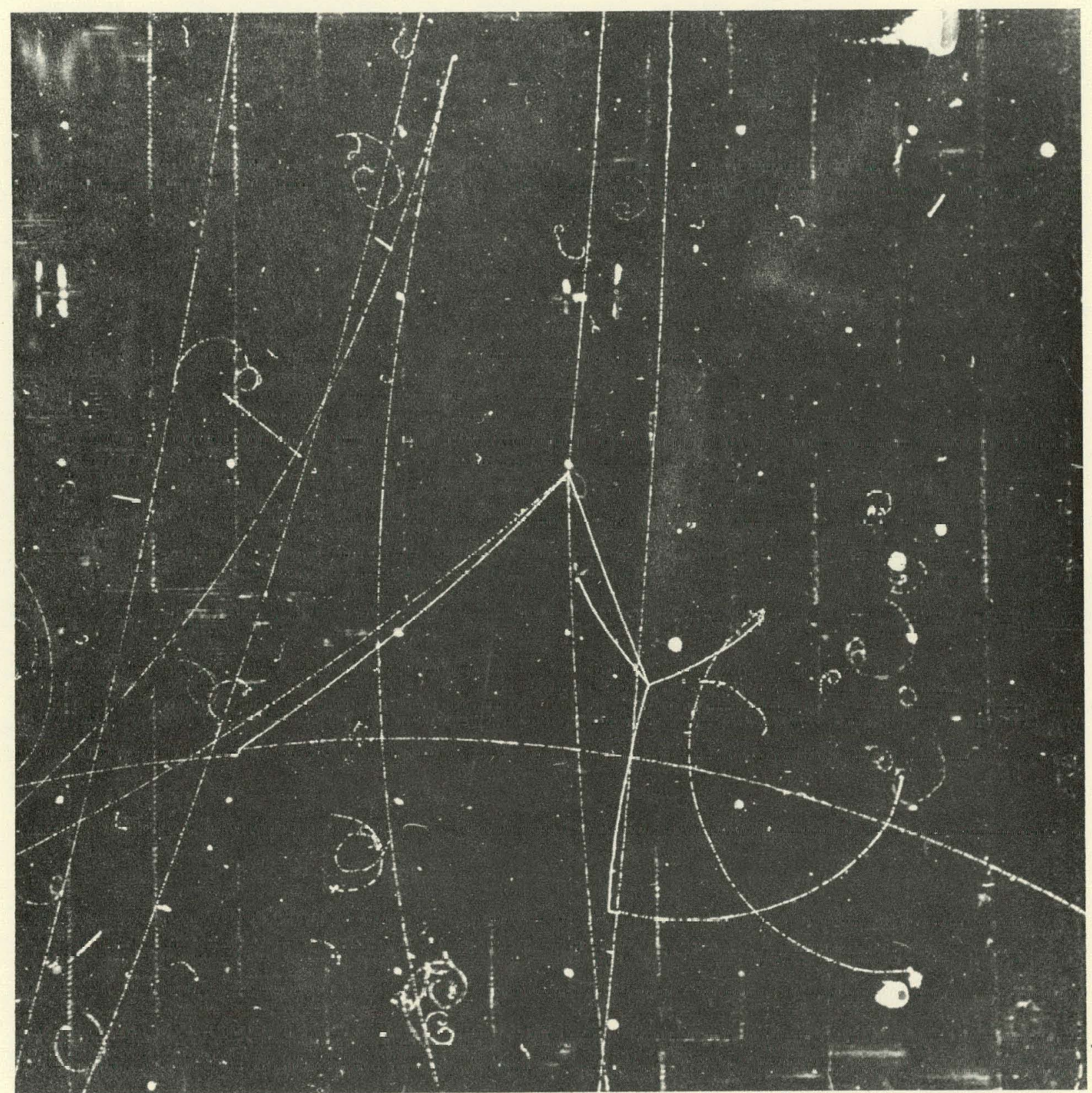

$\mathrm{ZN}-2464$

Fig. 7. An antiproton enters at the top and annihilates, producing two charged pions and $\mathrm{K}^{+}+\mathrm{K}^{-}$. The $\mathrm{K}^{+}$comes to rest, decaying into $\pi^{+}+\pi^{+}+\pi^{-}$. The $\dot{K}^{-}$comes to rest and interacts, producing a $\Sigma^{+}$and $\pi^{-}$. 


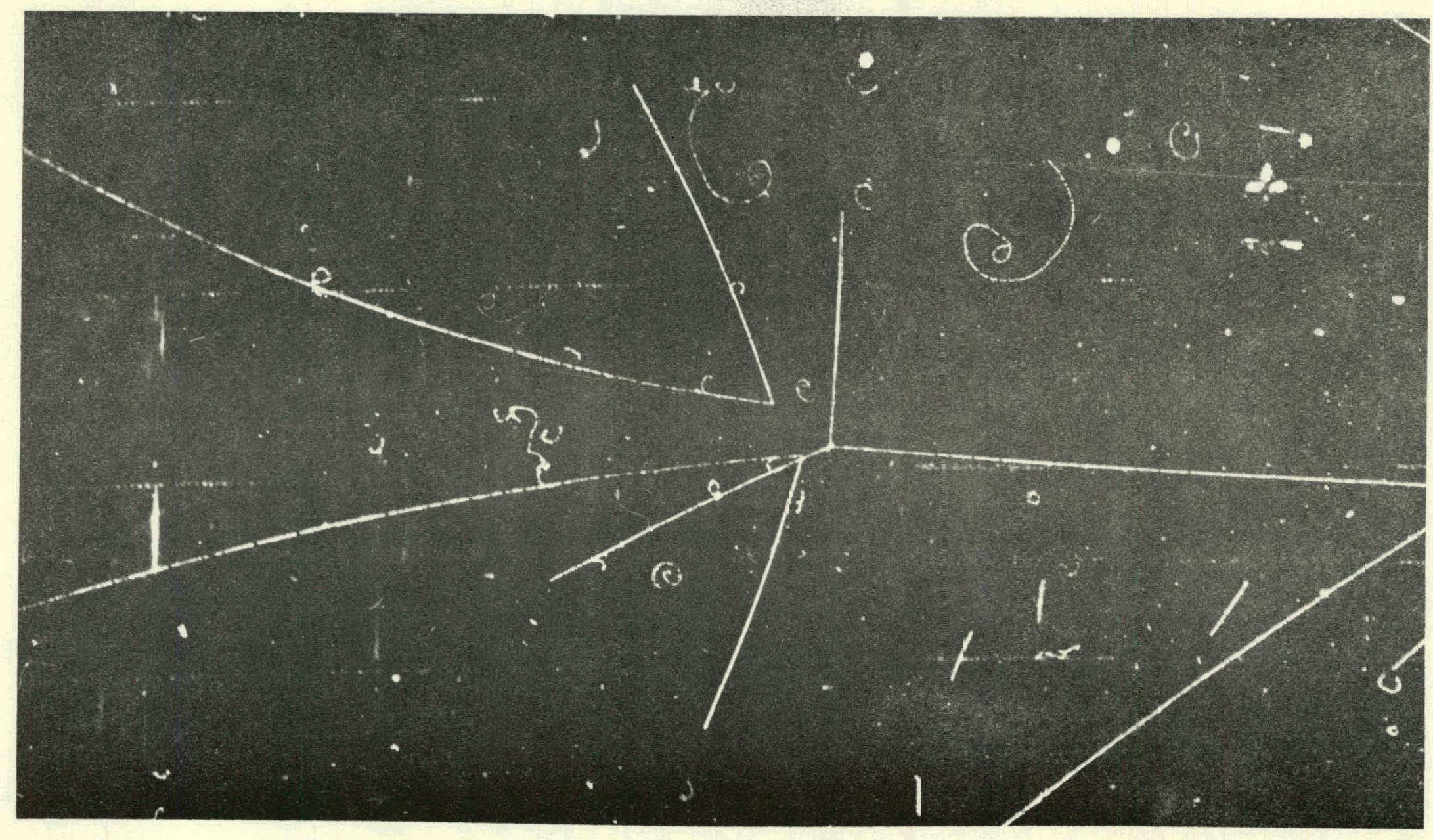

Fig. 8. An antiproton enters at the top and annihilates, producing two charged pions and $K^{0}+\bar{K}^{0}$, which are seen $t=$ decay irto pion pairs. 
We have investigated the hydrogen-like events with two $\pi^{ \pm}$for the number of events that satisfy the criteria for $\vec{p}+p \rightarrow 1 \pi^{+}+1 \pi^{-}$annihilation without producing any neutral pions. This was done by investigating (a) the angles between the pions in the center-of-mass system and (b) the $Q$ values ${ }^{7}$ of the charged pions for stars that emit $1 \pi^{+}+1 \pi^{-}+n \pi^{0}$. This analysis was confined to events where the momenta of both pions could be determined with sufficient accuracy. Hence the tracks had to (a) have a range of $8 \mathrm{~cm}$ of more before leaving the chamber, scattering, or interacting, or (b) come to rest in the chamber. The number of events satisfying these criteria correspond to a sample of $500 \mathrm{hydrogen-like} \mathrm{events.} \mathrm{In} \mathrm{this} \mathrm{sample} \mathrm{we} \mathrm{find} \mathrm{only} \mathrm{one} \mathrm{event}$ ( and even this event had a large error of $\sim 35 \%$ in the momentum measurement of a pion) that could satisfy the criteria for $\bar{p}+p \rightarrow 1 \pi^{+}+1 \pi^{-}$annihilation. This also is in agreement with the statistical-model multiplicity-distribution function, which predicts 0.6 such events per $500 \bar{p}-p$ annihilations.

\section{Neutral-Pion Production}

A. lower limit to the neutral -pion multiplicity can be obtained from the ouserved number of Dalitz pairs. We have observed 70 Dalitz pairs emitted from $3960 \bar{p}$ annihilation events. (No geometrical cutoffs were applied to this sample.) This sample does not include stars with observed strange particles. $\dot{A}$ Dalitz pair is formed in one out of $80 \pi^{0}$ decays. As high-energy Dalitz pairs can sometimes be mistaken for pion pairs, the number of neutral pions evaluated this way gives only a lower limit to the total number of neutral pions produced. We obtain $\left\langle\mathrm{N}_{\pi} 0\right\rangle \geq 1.4 \pm 0.2$ for $\overline{\mathrm{p}}$ annihilations in propane at 1050 $\mathrm{Mev} / \mathrm{c}$.

A more accurate estimate of the number of neutral pions can be obtained from the number of electron pairs produced by $\gamma$-ray conversion in the chamber. The mean free path for $\gamma$-ray conversion in propane is given in the Appendix of Agnew et al. as a function of the $\gamma$-ray energy. 20 The mean free path for conversion was evaluated by taking into account the energy spectrum of pions from the annihilation events. The fact that the energy spectra are different for different charged-pion multiplicities was also taken into account.

The average $\gamma$-ray path length in the chamber was evaluated from the geometry of the chamber. It was assumed that the angular distribution of the $\gamma$ rays in the chamber would be similar to that of the pions. Furthermore, it was assumed that only electron pairs with a projected length of $1 \mathrm{~cm}$ or 
more in the chamber would be detected. The effects of stray pairs pointing towards an antiproton star are negligible. The correction for pairs missed in both scans amounts to about $3 \%$. About $15 \%$ of the neutral pions are detected through the $\gamma$-ray conversion process.

The average number of neutral pions per star in hydrogen-like annihilation events is $\left\langle\mathrm{N}_{\pi} 0\right\rangle=1.64 \pm 0.15$. For $\overline{\mathrm{p}}$ stars in carbon, 32 we have $\left\langle N_{\pi} 0\right\rangle=1.54 \pm 0.10$. We note that $\left.\left\langle N_{\pi^{0}}\right\rangle\right) /\left\langle N_{\pi^{ \pm}}\right\rangle=0.49 \pm 0.05$ for the hydrogenlike events.

Tables VI and VII give the distribution of neutral pions for $\bar{p}$ stars in hydrogen and carbon. It was assumed that the annihilation stars in carbon that simulate $\bar{p}$ stars in hydrogen, have the same number of neutral pions for a given $\left\langle\mathrm{N}_{\pi} \pm\right.$ ) as the corresponding $\bar{p}$ stars in hydrogen.

4. Strange-Particle Production

a. Introduction. The nucleon-antinucleon annihilation process has been found to produce $4( \pm 1) \% \mathrm{~K}$-meson pairs per star for antiproton energies below $200 \mathrm{Mev}$. 19,20,21 Our experiment, at a higher energy ( 475 Mev), provides evidence for a rather rapid increase in the $\dot{K}$-meson production with energy. In subsection $4-\mathrm{g}$ we shall investigate the physical reason for this increase. Figures 7 and 8 give photographs of $\overline{\mathrm{p}}$ annihilation events with $\mathrm{K}$ mesons. The $\mathrm{K}$ mesons in both photographs interact in the bubble chamber.

b. Determination of detection efficiency. The 30-in. propane bubble chamber is highly efficient in detecting the short-lived $\mathrm{K}^{0}$ and hyperons. In the present experiment charged $\mathrm{K}$ mesons can be detected reliably only when they decay in the chamber. The hyperons are produced by $\overline{\mathrm{K}}$ absorption by a nucleon in the parent nucleus according to the reaction: $\overline{\mathrm{K}}+\mathrm{N} \rightarrow \cdot \pi+\mathrm{Y}$.

For the evaluation of the number of strange particles we chose:a central volume $(41$ by 26 by $8 \mathrm{~cm}$ ) of the chamber. Our acceptance criteria arethat both the annihilation and the $\mathrm{K}^{0}$ or $\Lambda$ decay occur in this volume. For the charged strange particles the acceptance criteria are that the decay or interaction occur in the visible region of the chamber (55 by 34 by $16 \mathrm{~cm}$ ).

$\mathrm{b}-1$. $\mathrm{K}^{0}$ mesons and $\Lambda$ hyperons. The decays of the $\mathrm{K}^{0}$ mesons and $\Lambda$ hyperons were identified by the measurement of their $Q$ values and coplanarity with respect to the antiproton star.

In the evaluation of the number of neutral strange particles we used the following facts: (a) Half of the $\mathrm{K}^{0}$ mesons are long-lived and escape the chamber 
The neutral-pion multiplicity as a function of the charged-pion multiplicity for $\bar{p}$ annihilation events in carbon.

\begin{tabular}{ccc}
\hline $\mathrm{N}_{\pi^{ \pm}}$ & $\frac{\text { Neutral pions per star }}{2.9+3.0}$ \\
\hline 0 & 0.9 \\
1 & $2.0 \pm 0.4$ \\
2 & $2.1 \pm 0.2$ \\
3 & $1.4 \pm 0.1$ \\
4 & $1.0 \pm 0.1$ \\
5 & $0.8 \pm 0.1$ \\
6 & $0 ! 7 \pm 0.3$ \\
7 & $1.0 \pm 0.8$ \\
\hline \hline
\end{tabular}

without detection; (b) of the remaining $\mathrm{K}^{0}$ mesons, $1 / 3$ have a neutral decay mode $\left(\pi^{0}+\pi^{0}\right)$ and hence escape detection; (c) $1 / 3$ of the lambdas also escape detection because of the neutral decay mode $\mathrm{N}+\pi^{0}$.

It was estimated that about $10 \%$ of the $\mathrm{K}_{1}^{0}$ mesons and lambdas would leave the fiducial volume. This estimate was evaluated from (a) the distribution in the chamber of the annihilation events, (b) the experimental angular distribution of the neutral $\mathrm{K}$ mesons and lambdas, (c) the range distribution of the neutral $\mathrm{K}$ mesons and lambdas as determined from their lifetimes and momentum distributions. A nearly equal fraction of the $K_{1}^{0} \cdots$ mesons and lambdas leave the fiducial volume; the fact that the halflife of the lambdas is longer is offset by their lower velocities.

$\mathrm{b}-2$. Charged $\mathrm{K}$ mesons. Among the charged $\mathrm{K}$-mesons we use only those that interact or decay in the chamber. For the evaluation of the identifiable fraction of $\mathrm{K}^{+}$mesons, we assume that the $\mathrm{K}^{+}$have the same momentum distribution as the neutral $\mathrm{K}$ mesons. Using the mean range of the $\mathrm{K}^{+}$mesons before they left the chamber, we evaluate the fraction coming to rest and the fraction decaying in flight in the chamber.

For $\mathrm{K}^{-}$mesons we likewise evaluate the fraction decaying in flight and those coming to rest and giving visible interaction products. 
:b-3. Charged hyperons We accept only the $\pi^{+}+n$ decay mode of the $\Sigma^{+}$, since the $p+\pi^{0}$ mode cannot be reliably distinguished from a proton scatter. A $\Sigma$ decay may sometimes be mistaken for a pion scatter if the track of either the decaying particle or decay product is steep.

c. $\mathrm{K}^{-}$meson contamination in the beam. Out of 118 stars producing identified strange particles, all except six stars had a visible-energy release consistent only with an incident antiproton. The remaining six stirs which gavo throo neutral $\mathrm{K}$ mesons and three hyperons could have been produced by a small admixture of $\mathrm{K}^{-}$mesons in the beam. We shall as sume that half of these six events are produced by $\mathrm{K}^{-}$mesons.

d. The number of strange particles. Table VIII gives a summary of our strange $\bar{i}$ particle events. In $3015 \bar{p}$ annihilations we have $229 \pm 31$ stars with strangepparticles, which leads to $\left\langle\mathrm{N}_{\mathrm{K} \overline{\mathrm{K}}}\right\rangle=8( \pm 1) \% \operatorname{per} \overline{\mathrm{p}} \operatorname{star}^{33}$ At low energies the:percentage of stars with strange particles was $4 \pm 1$.

Table IX gives the number of events with two strange particles observed in a single star. Better statistics is necessary to determine whether the " $\mathrm{K}^{+}+\mathrm{K}^{-}+\mathrm{n} \pi$ and the $\mathrm{K}^{0}+\overline{\mathrm{K}}^{0}+\mathrm{n} \pi$ modes prevail over the $\mathrm{K}^{+}+\overline{\mathrm{K}}^{0}+\mathrm{n} \pi$ and $\mathrm{K}^{0}+\mathrm{K}^{-}+\ddot{n} \pi$.

Table $\mathrm{X}$ gives the number of hydrogen-like annihilation events with strange particles. In Section II-B we determined that $16 \%$ of the hydrogen-' like stars with no observed $K$ mesons were fake $\bar{p}-H$ events: We assume that the same ratio also holds for stars with observed $K$ mesons. Then 66 events with strange particles in $\bar{p}-H$ stars are left. From these stars we deduce that $\left\langle\mathrm{N}_{\mathrm{K} \overline{\mathrm{K}}}\right\rangle=8( \pm 2) \%$ for annihilation events in hydrogen, and $\left\langle\mathrm{N}_{\mathrm{K} \overline{\mathrm{K}}}\right\rangle=$ $7( \pm 2) \%$ in carbon. From the number of hyperons we find that $28 \pm 6 \%$ of the $\overline{\mathrm{K}}$ mesons are absorbed.

e. The pion multiplicity in stars with $K$ mesons. In the analysis given in this section, we omit stars with hyperons, since such stars have a higher pion multiplicity because of the reaction $\overline{\mathrm{K}}+\mathrm{N} \rightarrow \pi+\mathrm{Y}$.

Figure 9 gives the observed charged-particle multiplicity distribution exclusive of identifiable protons and $K$ mesons. We will call this the apparent charged-pion multiplicity.

This apparent charged-pion multiplicity of 1.82 must be modified to obtain the correct pion multiplicity by taking into account the following facts: (a) Out of the total number of $\mathrm{K}^{-}$and $\mathrm{K}^{+}$mesons emitted, only $18( \pm 6) \%$ and $23( \pm 7) \%$ respectively have been identified as $\mathrm{K}$ mesons (see Table VIIi). 
Table VIII

Number of strange particles produced in $\overline{\mathrm{p}}$ annihilations in propane at a momentum of $1050 \mathrm{Mev} / \mathrm{c}$.

\begin{tabular}{|c|c|c|c|c|}
\hline Particle & No. observed & $\epsilon^{a}$ & $a^{b}$ & No. estimated \\
\hline $\mathrm{K}^{+}$ & $25 \pm 1$ & $0 .: 23 \pm 0.07$ & 1 & 109 \\
\hline $\mathrm{K}^{-}$ & $17 \pm 2$ & $0.18 \pm 0.06$ & 1 & 94.5 \\
\hline $\mathrm{K}^{0}$ or $\overline{\mathrm{K}}^{0}$ & $59 \pm 4$ & $0.90 \pm 0.03$ & 3 & 197 \\
\hline$\Sigma^{+}$ & $7.5 \pm 1.5$ & $0.90 \pm 0.05$ & 2 & 17 \\
\hline$\Sigma^{-}$ & $11.5 \pm 1.5$ & $0.90 \pm 0.05$ & 1 & 13 \\
\hline$\Lambda$ & $21 \pm 2$ & $0.90 \pm 0.05$ & 15 & 35 \\
\hline Total & $141 \pm 6$ & & & 465.5 \\
\hline
\end{tabular}

a. Here $\epsilon$ represents the correction factor for a $\mathrm{K}^{0}$ or $\Lambda$ leaving the fiducial volume, for a $\mathrm{K}^{+}$leaving the chamber, for a $\mathrm{K}^{-}$leaving the chamber or intëracting without visible prongs, for a $\Sigma$ being mistaken for a $\pi$, and for the detection efficiency of decays.

b. Here a represents the correction factor for (a) neutral decay modes, (b) the long-lived $\mathrm{K}_{2}^{U}$ mode, and (c) the $\Sigma^{+} \rightarrow \mathrm{p}+\pi^{0}$ decay mode.

\section{Table IX}

Number of events with two observed strange particles.

\begin{tabular}{cccccc}
\hline Particle & $\mathrm{K}^{-}$ & $\overline{\mathrm{K}}^{0}$ & $\Lambda$ & $\Sigma^{+}$ & $\Sigma^{-}$ \\
\hline $\mathrm{K}^{+}$ & 5 & 2 & 6 & 0 & 0 \\
$\mathrm{~K}^{0}$ & 1 & 6 & 2 & 1 & 3 \\
\hline
\end{tabular}

Table X

Number of strange particles in hydrogen-like events.

\begin{tabular}{|c|c|c|c|c|}
\hline Particle & No. observed & $\epsilon^{a}$ & $a^{-b}$ & No. estimated \\
\hline $\mathrm{K}^{+}$ & $8.5 \pm 0.5$ & $0.23 \pm 0.07$ & 1 & 37 \\
\hline $\mathrm{K}^{-}$ & $7.5 \pm 0.5$ & $0.18 \pm 0.06$ & 1 & 42 \\
\hline $\mathrm{K}^{0}$ or $\overline{\mathrm{K}}^{0}$ & $23 \pm 2$ & $0.90 \pm 0.03$ & 3 & 77 \\
\hline Total & $39 \pm 3$ & & & 156 \\
\hline
\end{tabular}

\begin{tabular}{l}
${ }^{\mathrm{a}}$ See note $\mathrm{a}, \mathrm{T}$ Table VIII. \\
$\mathrm{b}$ See note b, Table VIII. \\
\hline
\end{tabular}




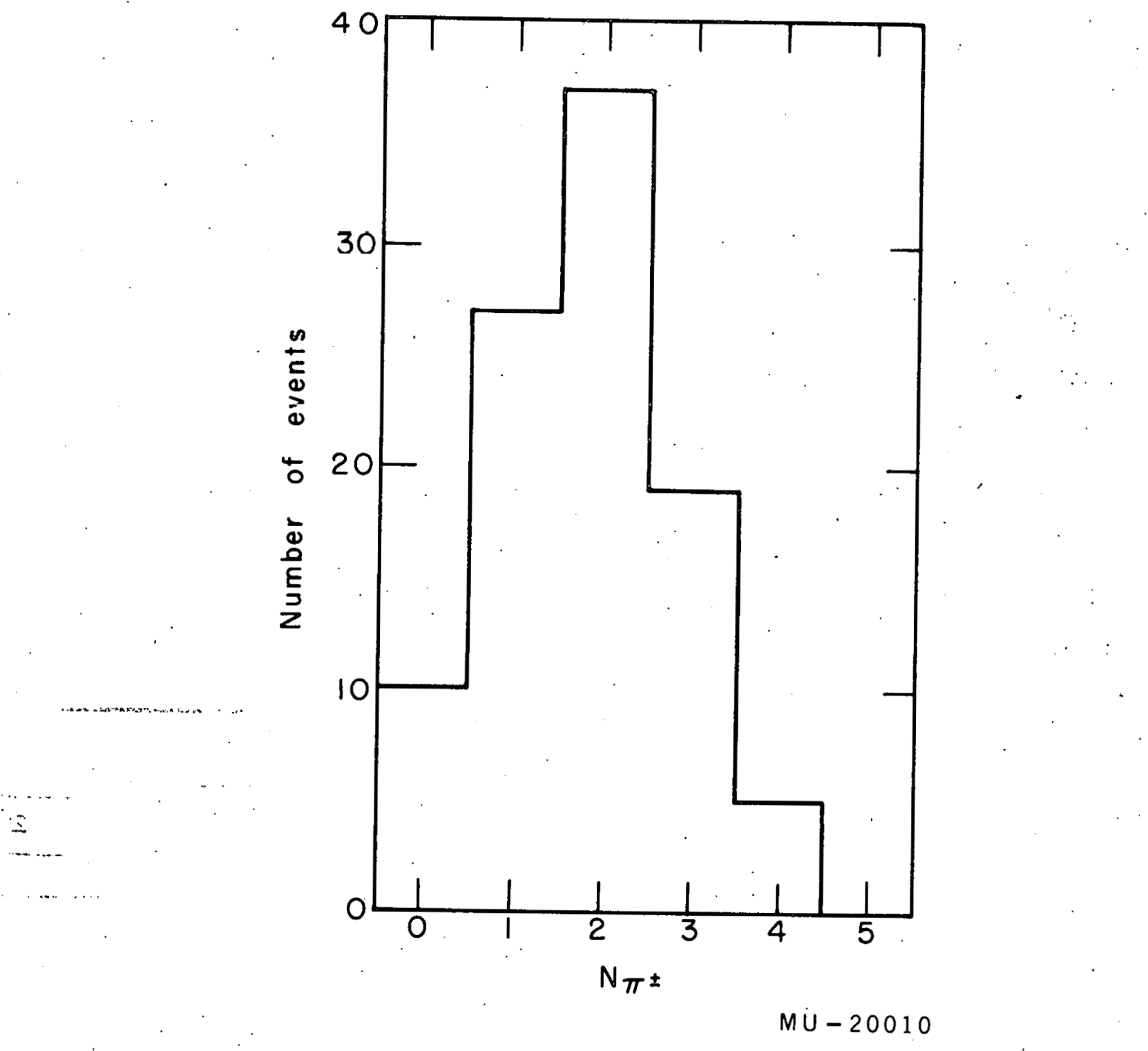

Fig. 9. The observed apparent charged-pion mulitplicity distribution from $\bar{p}$ annihilation stars giving $K$ mesons Events with observed hyperons have been excluded. The corrections to be applied to this distribution are described in Section II-D-4-e. 
The majority of the remaining $K$ mesons have been included among the $\pi$ mesons. This leads to a correction reducing the $\pi^{ \pm}$multiplicity by 0.35 . (b) Nine percent of the pions produced in this sample are absorbed and a correction for this fact raises the multiplicity. ${ }^{34}$. Making both corrections we obtain $\left\langle\mathrm{N}_{\pi}\right\rangle_{\mathrm{K}}=1.6 \pm 0.30$ for the primary multiplicity of charged pions in $\overline{\mathrm{p}}$ annihilation stars with $\mathrm{K}$ mesons. Assuming the number of neutral pions to be half that of the charged pions, we deduce $\left\langle N_{\pi}\right\rangle_{K}=2.4 \pm 0.5$.

f. Momentum sprectra. Figures 10 and 11 give the experimental momentum spectra in the c.m. system for the $\mathrm{K}^{0}$ and $\pi^{ \pm}$mesons from stars where. $\mathrm{K}^{0}$ mesons are produced $\left(\left\langle\mathrm{P}_{\mathrm{K}}\right\rangle_{\mathrm{c} \cdot \mathrm{m} .}=433 \pm 60 \mathrm{Mev} / \mathrm{c}\right.$ and $\left\langle\mathrm{P}_{\pi^{ \pm}}\right\rangle_{\mathrm{c} \cdot \mathrm{m}}=$ $414 \pm 50 \mathrm{Mev} / \mathrm{c})$. The experimental spectra are compared with those calculated on the basis of therstatistical model. The calculations were performed for annihilations with one, two, three, or four pions produced in addition to the $\mathrm{K}$ meson pair. The latter momentum distributions were calculated by Dr. T.F. Hoang. ${ }^{35}$ His calculations make use of Lorentz-invariant phase space as discussed by Srivastava and Sudarshan ${ }^{36}$ and Desai. 29

We note that the average momenta of particles leaving the interaction volume appear to be independent of the nature of the particles (i.e., K or $\pi$ meson). The average momenta also appear to be independent of whether only pions are produced on annihilation, or both $\mathrm{K}$ and $\pi$ mesons are produced.

g. Theoretical analysis of $\mathrm{K}$ meson production in antiproton annihilation. It is the purpose of this section to investigate whether a simple modification of the usual Fermi statistical model could explain the amount of $\mathrm{K}$-meson production and the increase of the $\mathrm{K}$-meson production in $\overline{\mathrm{p}}$ annihilation events with increasing $\bar{p}$ energy.

Customarily a single parameter, the interaction volume, has been used for the calculation of both the $\pi$ - and $K$-meson production. The transition probability for $n \pi$ and two $K$ mesons in $\bar{p}-\mathrm{p}$ annihilation can be written as $37,1^{\prime} 0,36$

$$
S_{n, 2}=A \frac{\frac{1}{2}\left[g_{n, 2}(1)+g_{n, 2}(0)\right]}{n !(1 !)^{2}} \frac{\left(M_{K} \Omega_{K}\right)^{2}\left(\mu \Omega_{\pi}\right)^{n}}{(2 \pi)^{3}\left(2+n_{1}\right)} \cdot F_{n, 2}\left(W_{0}{ }^{2}\right) .
$$

Here $g_{n, 2}(I)$ is the I-spin weighting factor for $n \pi$ and two $K$ mésons, and $\mathrm{F}_{\mathrm{n}, 2}\left(\mathrm{~W}_{0}{ }^{2}\right)$ is given by 


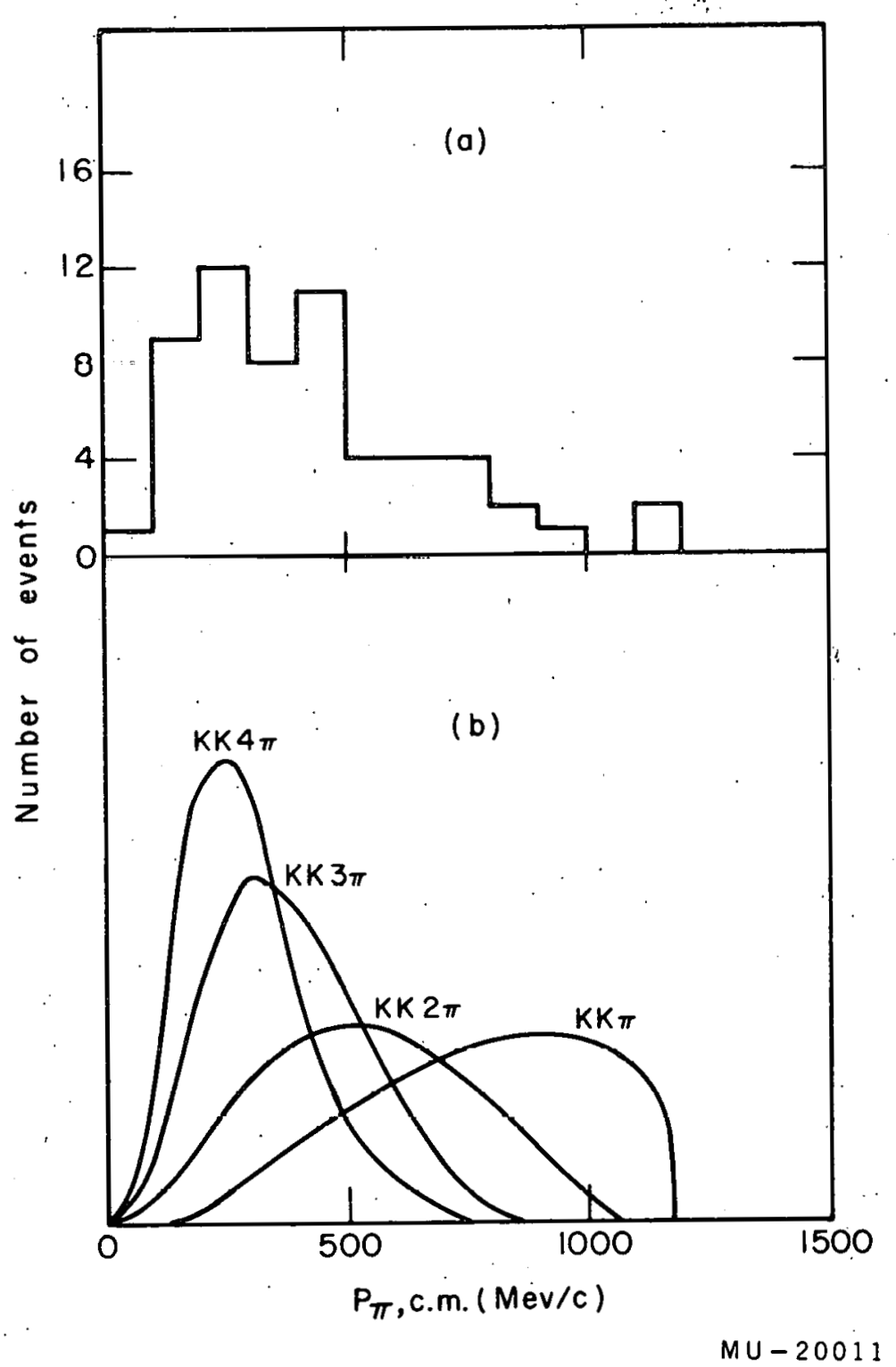

Fig. 10. (a) The pion momentum spectrum associated with strange particles in $\bar{p}$ annihilation. (b) The corresponding pion momentum spectra evaluated by Hoang. 


\section{iso:}

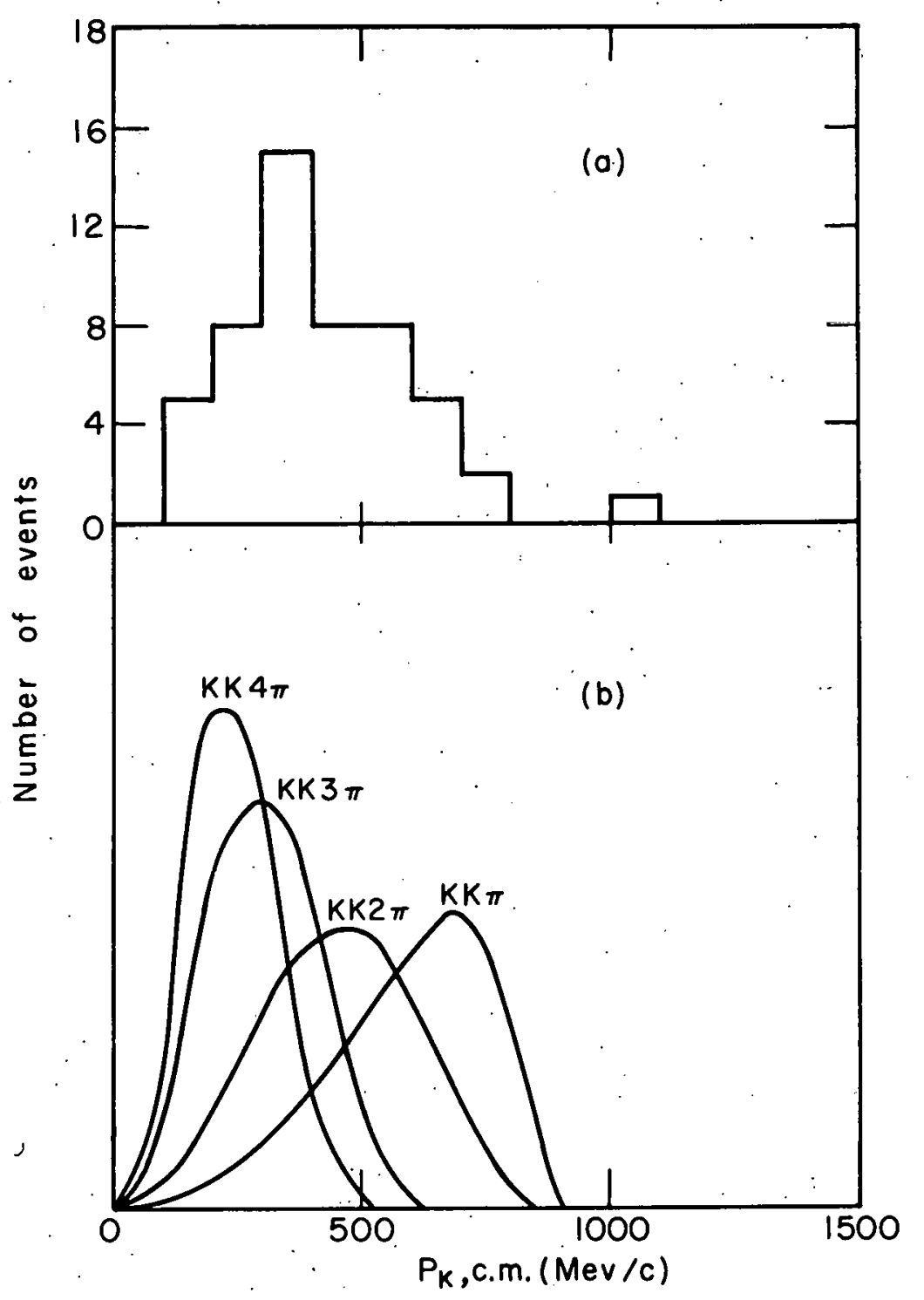

$M U-20012^{\circ}$

Fig. 11. (a) The momentum spectrum of neutral $\mathrm{K}$ mesons in the $c_{0} m_{0}$ system from $\bar{p}$ annihilation stars. (b) The K-meson momentum spectra evaluated by Hoang. 
$F_{n, 2}\left(w_{0}^{2}\right)=(\ddot{4} \pi)^{n} \int_{\mu}^{\omega} 1 \max \int_{\mu}^{\omega_{2} \max } \cdots \int_{\mu}^{\omega_{n} \max } \overbrace{i=1}^{n} P_{i} F_{2}\left(w_{n^{i}}^{2}\right) \prod_{i=1}^{n} d \omega_{i}$.

where we have

$$
\begin{aligned}
& P_{i}^{2}+\mu^{2}=\omega_{i}^{2} \\
& F_{2}\left(W_{n}^{2}\right)=2 \pi\left(1-\frac{4 M_{K}^{2}}{\bar{W}_{n}^{2}}\right) 1 / 2, \\
& w_{i}^{2}=W_{i-1}^{2}-2 W_{i-1} \omega_{i \cdot}+\mu_{i}^{2} ; \\
& \Omega_{\pi}=\lambda \frac{4 \pi}{3} \frac{1}{\mu^{3}} .
\end{aligned}
$$

in units of $\hbar=c=1$ and

$$
\omega_{i \max }=\frac{w_{i-1}{ }^{2}+\mu^{2}-\left[(n-i) \mu+2 M_{K}\right]^{2}}{2 W_{i-1}} \text {. }
$$

Here $W_{0}$ is the annihilation energy in the center-of-mass system, $\mu$ and $M_{K}$ are the $\pi$ - and $K$-meson rest masses, respectively, and $P_{i}$ and $\omega_{i}$ are the momentum and the energy, respectively, of the ith pion. 38

Fur $\Omega_{I S}-\left(\mu / M_{K}\right) \Omega$ we get the single-parametcr model, that is $S_{n, 2}$ is proportional to $\left(\mu \Omega_{\pi}\right)^{n+2} \cdot$ The results obtained from the above calculation, which adjusted the parameter $\lambda$ to fit the mean pion multiplicity, failed to agree with the experimentally determined $\mathrm{K}$-meson production for. $\Omega_{K}=(\mu / M) \Omega_{\pi}$. In our attempt to fit the $\mathrm{K}$-meson production, we introduced one additional parameter $\xi=\Omega_{K} / \Omega_{\pi^{\circ}}$. Different coupling constants of the pion and. $\dot{K}$ meson as well as the difference in their Compton wave lengths could be a theoretical justification for introducing the new parameter. It should be noted here, however, that such a treatment has to be considered as a phenomenological fit rather than a profound theory that would determine the relative strength of interactions of pions and $\mathrm{K}$ mesons.

With this two-parameter statistical model, we calculated the increase in $\mathrm{K}$-meson production as a function of the annihilation energy. The results of these calculations are summarized in Tables XI and XII. These tables have 
been evaluated on the basis of the Fermi statistical model with Lorentz- invariant phase space and different interaction - volume parameters $\Omega_{\pi}$ and $\Omega_{\mathrm{K}}$. Here $\Omega_{\pi}$ was adjusted so as to obtain $\left\langle\mathrm{N}_{\pi}\right\rangle=4.9$ for annihilations at rest and $\Omega_{K}$ was adjusted by fitting $\xi$ so as to obtain $4 \%$ (see Table XI,..column 4 ) or 5\% (see column 5) $\mathrm{K}$-meson production for annihilations at rest. This was done by setting $\Omega_{\pi}=8 \Omega_{\pi}^{0}$ and $\xi=0.10$ and 0.11 , respectively. Table XI gives (a) the fraction of stars with strange particles for $\bar{p}-p$ annihilations as a function of $W_{0}$ and $(b)$ the average number of pions associated with $\mathrm{K}$ mesons. The corresponding pion multipliçity distributions are given in Table XII.

We also calculated $\mathrm{K}$-meson production and the $\left\langle\mathrm{N}_{\pi}\right\rangle_{K}$ value for antiproton annihilations in carbon, and found no significant differences from $\overline{\mathrm{p}}-\mathrm{H}$ annihilation.

We note that the statistical model leads to a rapid increase in the $\pi-$ meson multiplicity in annihilation events where $\mathrm{K}$ mesons are produced. At our energy, $W_{0}=2100 \mathrm{Mev}$, the predicted value of $\left\langle\mathrm{N}_{\pi}\right\rangle_{\mathrm{K}}=2.4$ is in good agreement with our experimental value of $2.4 \pm 0.5$. The present data on $\left\langle\mathrm{N}_{\pi}\right\rangle_{\mathrm{K}}$ for annihilations at rest are statistically poor, but they are consistent with the calculated value. Future experiments will have to verify. the expected increase in $\left\langle N_{\pi}\right\rangle_{K}$ with increasing annihilation energy.

A comparison of the calculated $\mathrm{K}$-meson-production increase with the experimental data shows, however, that only in the limits of the RMS errors quoted can we get agreement with our calculation. It is clear that until we solve our fundamental difficulty in calculating the pion multiplicity with a reasonable interaction volume, all attempts to fit $\mathrm{K}$-meson production are of a very preliminary nature.

In terms of the Koba-Takeda model, we have to consider the restric: tions placed on $\mathrm{K}$-meson production by the available energy in the nucleonantinucleon cores. In this model, the states with two or three pions in the pion cloud of the $\bar{p}-\mathrm{N}$ system strongly prevail over the states with no or one pions. With no or one pions in the cloud, the restriction of energy conservation does not in general restrict the production of $\mathrm{K}$ mesons in the annihilation of the cores. The contribution of states having two pions in the cloud is restricted, however. It is even more restricted for states having three or more pions in the cloud. With increasing annihilation energy, however, the energy of the 
Table XI

Fraction of stars having strange particles in $\bar{p}-p$ annihilation as a function of $\mathrm{W}_{0}$, the annihilation energy in the center-of-mass system.

\begin{tabular}{|c|c|c|c|c|c|}
\hline $\mathrm{P}_{\overline{\mathrm{P}}}(\mathrm{Mev} / \mathrm{C})$ & $\mathrm{W}_{0}(\mathrm{Mev})$ & $\begin{array}{l}\text { \% stars with } \mathrm{K} \\
\text { mesons (expt) }\end{array}$ & $\begin{array}{l}\% \text { star } \\
\text { mesor }\end{array}$ & $\begin{array}{l}\text { with } K \\
\text { (calc) }\end{array}$ & $\left\langle N_{\pi}\right\rangle_{K}$ \\
\hline 0 & 1876 & $4.0 \pm 1.0$ & $4.0^{a}$ & $5.0^{b}$ & 1.9 \\
\hline$\therefore 1070^{\circ}$ & 2100 & $8.0 \pm 1.0$ & $5.6 \cdots$ & 7.0 & $\ldots 2.1$ \\
\hline 2230 & 2510 & & 8.3 & 10.4 & 3.1 \\
\hline
\end{tabular}

Normalized to $4 \% \mathrm{~K}$-meson production for annihilations at rest.

h. Normalized to $5 \% \mathrm{~K}$-meson production fur dnnililations at rest.

\begin{tabular}{|c|c|c|c|c|c|c|}
\hline \multicolumn{5}{|c|}{ Table XII } & & \\
\hline \multicolumn{5}{|c|}{ Associated pion multiplicity distributions ${ }^{a}$} & . & $\cdots$ \\
\hline \multirow[b]{2}{*}{$\mathrm{P}_{\overline{\mathrm{p}}}(\mathrm{Mev} / \mathrm{c})$} & \multicolumn{4}{|c|}{$\%$ stars with $2 \mathrm{~K}$ and } & & \\
\hline & $0 \pi$ & $1 \pi$ & $2 \pi$ & $3 \pi$ & $4 \pi$ & \\
\hline 0 & 1 & 25 & 55 & 18 & 1 & \\
\hline 1070 & 0 & 11 & 46 & 37 & 6 & \\
\hline 2230 & 0 & 2 & 20 & 46 & 32 & \\
\hline
\end{tabular}

Production of tive pions has beèn néglected. 
cores increases, and the contribution of states with a higher pion multiplicity in the cloud increases. Hence the Koba-Takeda model also leads to (a) an increase in the $\mathrm{K}$-meson multiplicity, and (b) an increase in the multiplicity of the $\pi$ mesons associated with $\mathrm{K}$ mesons with increasing annihilation energy. Crude calculations indicate that the increase in the above quantities is of a similar order of magnitude as that predicted by the Fermi statistical model.

\section{E. Nuclear Excitation in Carbon}

In the case of annihilations in carbon nuclei we have the ratio $N / P=1.1 \pm 0.1$ among the knock-on nucleons. (There is an excess of $\pi^{-}$produced on annihilation, and neutron knock-on emission is preferred in the case of $\pi^{-}$absorption). We have not carried out a determination of the energy spectrum of knock-on protons in the propane bubble chamber experiment. The following is hence an indirect order-of-magnitude estimate of the knock-on nucleon energies per annihilation in carbon.

Our data in paper II and in $3 x$ gel emulsions show that the average energy of knock-on protons does not vary appreciably with the nuclear mass. This conclusion is also consistent with the propane bubble chamber experiment of Agnew et al. 20 The proton multiplicity per carbon star is $2.18 .^{39}$ Assuming that our proton energy spectrum has the same fraction of knock-on protons as Agnew's, 20 we deduce a knock-on proton multiplicity of $1.5 \pm 0.2$ per star in carbon. The average energy at which the pions interact is evaluated in Appendix A. We estimate that because of the higher energy of the interacting pions, the average energy of the knock-on protons is higher by about $20 \mathrm{Mev}$ than in experiments at low (at rest or at $150 \mathrm{Mev}$ ) $\overline{\mathrm{p}}$ energy. The energy given to knock-on nucleons was thus found to be $\left\langle E_{k 0}\right\rangle=380 \pm 60 \mathrm{Mev}$.

For annihilations in carbon at an average antiproton momentum of 1050 $\mathrm{Mev} / \mathrm{c}$ the average evaporation energy is $60: \pm 30 \mathrm{Mev}$. The method of evaluating this is described in a later section dealing with nuclear evaporation in emulsions. Since the above value is derived by extrapolating the evaporation theory to a region where it is not applicable, the error in the above quantity is large.

The average energy per star for the emission of nucleons or fragments is $U=440 \pm 70 \mathrm{Mev}$. This value, together with the data of Appendix A gives us $0.9 \pm 0.15$ as the average number of pions absorbed per star in carbon. The primary pion multiplicity for antiproton stars with no strange,particles in carbon at $1050 \mathrm{Mev} / \mathrm{c}$ is thereby $\left\langle\mathrm{N}_{\pi}\right\rangle=5.0 \pm 0.2$. 


\section{III: THE EMULSION EXPERIMENT}

\section{A. Introduction}

In this experiment we chose to use emulsions diluted with excess gelatin (3xgel) in order to obtain more abundant data on $\bar{p}-\mathrm{H}$ interactions and to determine separately the annihilation cross sections with the heavy. (Ag, Br) and light $(\mathrm{C}, \mathrm{O}, \mathrm{N}$,$) nuclei in emulsions. It was decided to analyze only the$ $\overline{\mathrm{p}}$ annihilations in flight because the difference between annihilations with light and heavy nuclei was expected to be most pronounced in this case.

Our present investigations further confirm that the energy and angular dependence of the $\bar{p}-p$ elastic-scattering cross section agrees with the theoretically estimated values of Ball, Chew and Fulco. ${ }^{3-5}$ The energy dependence of the annihilation mean free path in emulsions in the energy interval from 10 to $250 \mathrm{Mev}$, and the annihilation cross sections with the light and heavy emulsion nuclei has been determined.

We have determined the nuclear excitation in $3 x g e l$ emulsions, and found the average energy per prong of the knock-on and evaporation protons to be independent of the nuclear composition. The angular distribution of the knock-on protons relative to the incoming antiproton has been found to depend strongly on the energy of the knock-on protons. The fraction of low-energy alpha particles among the heavy prongs has been found to be considerably higher in the case of $\bar{p}$ annihilations in the light emulsion nuclei.

\section{B. Experimental Procedure}

\section{The Antiproton Beam}

The purification of the beam for our emulsion experiment was achieved by a momentum separation of the antiprotons relative to the background particles. An absorber; a lead collimator, and a subsequent partial directional separation by a bending magnet were used. The chief difference of the present exposure from that described in paper II is the addition of a: second absorber followed by subsequent momentum separation. The ratio of antiprotons to background particles (mostly $\mu$ mesons produced by pion decay) was thus improved by a factor of 50. The momentum of the beam at the stack was $730 \mathrm{Mev} / \mathrm{c}$.

The arrangement of the apparatus and the description of the beam has been presented by Agnew et al. 20 The emulsion experiment differed from the bubble chamber experiment by the introduction of a bending magnet just in 
front of the emulsion stack. This magnet was used to get rid of possible protons in the beam, which would be hard to distinguish from antiprotons at the entrance of the emulsion stack.

\section{Scanning and Measurements .}

In the emulsion plates the scanning proceeded parallel to the leading edge of the stack, at a distance of $5 \mathrm{~mm}$ from the edge. The antiproton tracks were distinguished from the large background of particles (about $10^{4}$ for one antiproton) at minimum ionization through a grain density approximately twice minimum. As the beam was well-collimated, only tracks within $10 \mathrm{deg}$ of the beam direction were followed as prospective ántiprotons, until they came to rest or interacted in flight.

Only the annihilation stars that occurred in flight--i.e. above 10-Mev kinetic energy--were analysed in this work. Here we were primarily inter= ested in studying the nuclear excitation. Therefore only prongs that could be protons-i.e. those with a grain density of 1.5 times minimum or greater-were followed out. The ranges of all these prongs were measured and their cnd points were examined for possible decay secondaries. Identification was done by ordinary emulsion techniques, and the low ionization in the $3 x g e l$ emulsions permitted grain counts on tracks up to 6 times minimum ionization.

All stars were examined by three persons to find a greater proportion of the minimum-ionization tracks. The projected and dip angles we're measured on all tracks.

3. Proton Contamination

A combination of range and ionization measurements permitted antiprotons to be distinguished from particles of different mass, but a small background of protons still remained. The tracks that could not at first be definitely identified as antiprotons were further analysed by having their endpoints examimed for minimum-ionization tracks, and in the case of stars in flight, for total-energy release. From the distribution in angle, range, grain density versus mean track length, etc, of the identified $\bar{p}$ it was possible to determine the nature of the remaining unidentified tracks. The results are presented in Tiable XIII.

\section{Antiproton Cross Sections}

1. Antiproton-Proton Ela'stic Scattering

Ball and Chew have given a model for the antinucleon-nucleon interaction, 
in which they have used the Signell-Marshak-Gartenhaus nucleon-nucleon potential. ${ }^{3}$ They have modified it to change the sign of the one-pion exchange potential and to change the reflecting inner bourdary into an absorbing one, using approximations that limit the validity of the model to an antiproton energy from 50 to $260 \mathrm{Mev}$. Fulco and Ball have applied the above model to antiproton-proton elastic scattering. 4,5

The present experiment gave $17 \overline{\mathrm{p}}-\mathrm{H}$ elastic scattering events. Table XIV summarizes the world data on the energy dependence of the antiproton-proton elastic-scattering cross section as obtained from emulsions. The criteria used in identifying $\overrightarrow{\mathrm{p}}-\mathrm{H}$ elastic-scattering events have already been described els e.where. 40,41

In the propane bubble rhamber experiment of Agnew et al. , ${ }^{20} 42 \bar{p}-p$ elastic scatters have been observed in the corresponding encrgy interval, giving a cross section of $62 \pm 12 \mathrm{mb}$. The combined emulsion and bubble chamber data give a cross section of $59 \pm 6 \mathrm{mb}$ at an average energy of about $145 \mathrm{Mev}$.

Coombes et al. ${ }^{24}$ in a recent counter experiment have found the $\overline{\mathrm{p}}-\mathrm{p}$. elastic-scattering cross section to be $72+9 \mathrm{mb}$ at $\mathrm{T}_{\mathrm{p}}=133 \mathrm{Mev}$ and $64+7$ $\mathrm{mb}$ at $\mathrm{T}_{\overline{\mathrm{p}}}=197 \mathrm{Mev}$. Their angular distribution is in agreement with that of the Ball-Chew-Fulco theory, but they were not able to measure the backwardscattering cross section at this energy.

Figures 12 and 13 give the combined emulsion and bubble chamber data on the energy dependence of the $\bar{p}-p$ elastic scattering cross section and the angular distribution of $\bar{p}-p$ elastic scatterıng, respeclively. Tle agreemcrit with the Ball-Chew-Fulco theory is good. Fulco predicted about $7 \%$ scattering in the backward direction in the center-of-mass system. We observe $8( \pm 4:) \%$. 2. Annihilation Cross Sections with Complex Nuclei

We have now a sufficient number of events for determining the energy dependence of the annihilation cross section on complex nuclei. From a comparison of the lxgel and 3xgel emulsion data, it is also possible to evaluate separately the cross sections for the light and heavy nuclei.

Table XV gives a summary of the data on various interactions and on the path length, using the most recent data at Berkeley as well as those of $A C E$ and II.

The energies of antiprotons interacting in flight were determined by one or more of the following three methods: (a) the estimated residual range, 
Table XIII

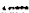

Classification of 132 events in emulsions out of a total of 716 events that were not identified as antiprotons in the first scan.

\begin{tabular}{lcc}
\hline & $\bar{p}$ & $p^{+}$ \\
Individually identified & - & 67 \\
Over side & 18 & $1 \pm 1$ \\
Over edge or into hole & $5 \pm 1$ & $4 \pm 3$ \\
Disappearances in flight & $7 \pm 3$ & $1+2$ \\
Small stars in flight & $7+1$ & $8 \pm 2$ \\
P & $3 \pm 2$ & $3 \pm 2$ \\
\hline Total & $8+2$ & $84 \pm 5$ \\
\hline \hline
\end{tabular}


Table XIV

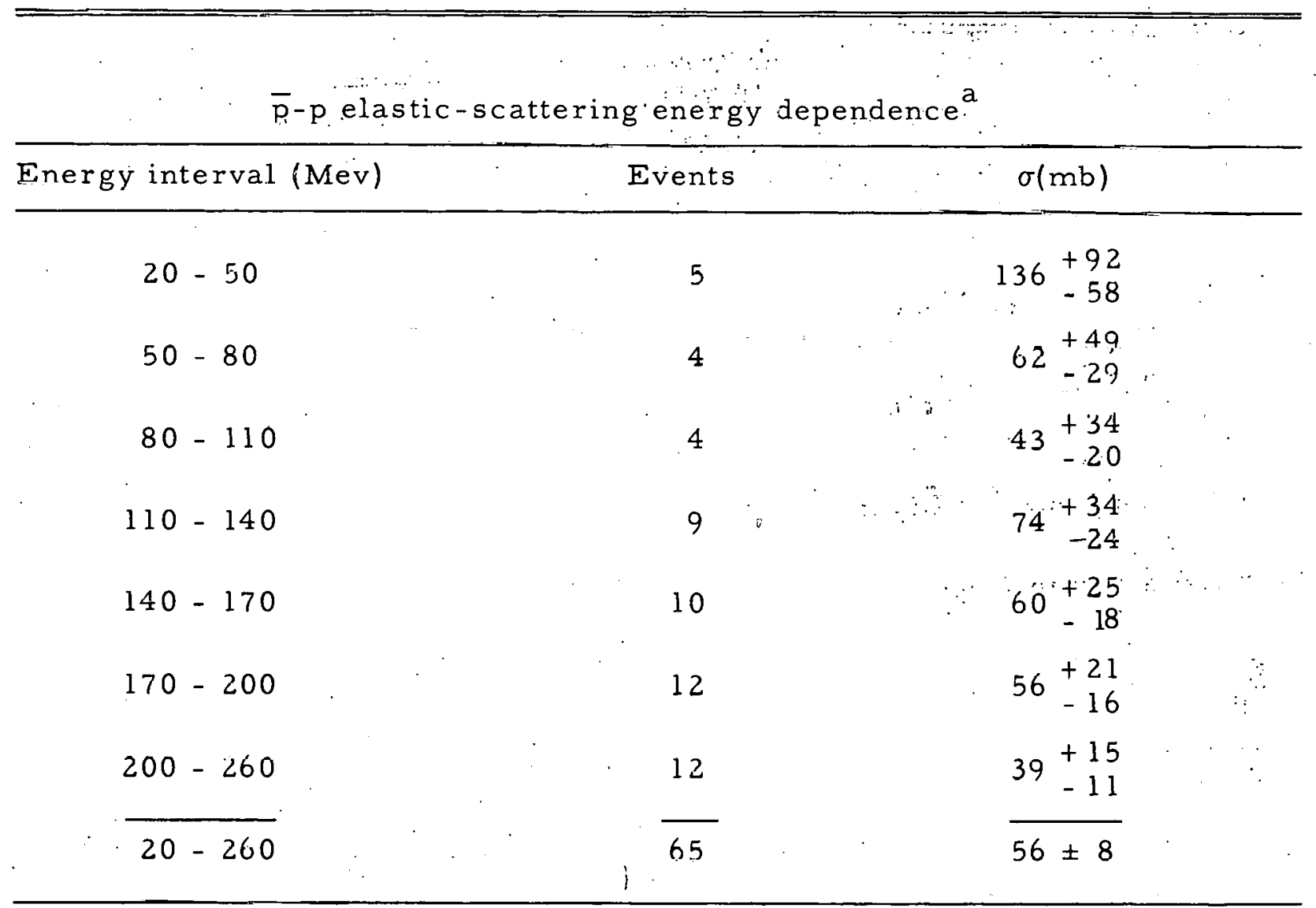

${ }^{a}$ The above table is compiled from the published data and private communications from:

(i) G. Goldhaber, T. Kalogeropoulos, and R. Silberberg, Lawrence Radiation Laboratory, University of California, Berkeley, Calif.

(2) A. Armstrong and G. Frye, Los Alamos Scientific Laboratory, Los Alamos, New Mexico.

(3) A. Engler, P.B. Jones, and.J.H. Mulvey, Oxford University, England.

(4) E. Amaldi, G. Barone, G. Bellettini, C. Castagnoli, M. Ferro-Luzzi, and A. Manfredini, Institute of Physics, Rome, Italy.

(5) A. Berthelot and F. Lévy, Center for Nuclear Studies, Saclay, Fränce.

(6) A. G. Ekspong and B.E. Ronne, University of Uppsala, Uppsala, Sweden. 
(b) ionization measurements, (c) multiple-scattering measurements. The first method was used above an energy of about $140 \mathrm{Mev}$, the second method between 40 and $400 \mathrm{Mev}$, and the third method at lower energies. All antiprotons that seemed to come to rest were analysed by multiple-scattering measurements, by which interactions in flight could be identified down to an energy of $10 \mathrm{Mev}$.

Figure 14 gives the energy dependence-of the annihilation mean free path of antiprotons in lxgel and $3 x g e l$ emulsions combined, weighted according to the respective path lengths.

The average mean free path for $\bar{p}$ annihilation was $18.9 \pm 1.5 \mathrm{~cm}$ in lxgel and $23.1 \pm 1.4 \mathrm{~cm}$ in $3 \times g e l$ emulsions. Including the data from Rome 21 and Uppsala 37 makes the average annihilation mean free path in emulsions $19.2 \pm 0.8 \mathrm{~cm}$.

From the mean free paths and the known concentrations of elements in $3 x g e l$ and lxgel emulsions, it was possible to evaluate the average annihilation'cross section in either kind of emulsion. The average cross sections were adjusted so as to exclude the antiproton-hydrogen annihilation events, by considering $5 \%$ of the annihilations in flight in lxgel and $7.5 \%$ in $3 \times g e l$ to be $\bar{p}-\mathrm{H}$ annihilations. The above estimates were arrived at by using the $\bar{p}-\mathrm{p}$ annihilation cross sections as determined by Coombes et al. 24 the concentration of hydrogen in emulsions, and the total $\bar{p}$ path length in emulsions. This is also consistent with our data in $3 \times g e l$, where we obtained $17 \pm 5$ as the lower limit of $\overrightarrow{\mathrm{p}}-\mathrm{H}$ annihilations in a sample of 215 stars that were more than $25 \mu$ from the emulsion surface.

From the following equations it was then possible to evaluate the average antiproton-annihilation cross sections in silver bromide and in gelatin with hydrogen excluded:

$$
\begin{aligned}
& \sigma_{1}^{a}=\sigma_{\ell}^{a} f_{1 \ell}+\sigma_{h}^{a} f_{1 h} \\
& \sigma_{3}^{a}=\sigma_{\ell}^{a} f_{3 \ell}+\sigma_{h}^{a} f_{3 h}
\end{aligned}
$$

Here $\sigma^{a}$ is the average annihilation cross section, $h$ and $\rho$ stand for the heavy and light elements in emulsion, respectively (with hydrogen excluded), 1 and 3 stand for lxgel and $3 x$ gel emulsions, respectively, and $f$ is the fraction of atoms in emulsions with hydrogen excluded. 
Table XV

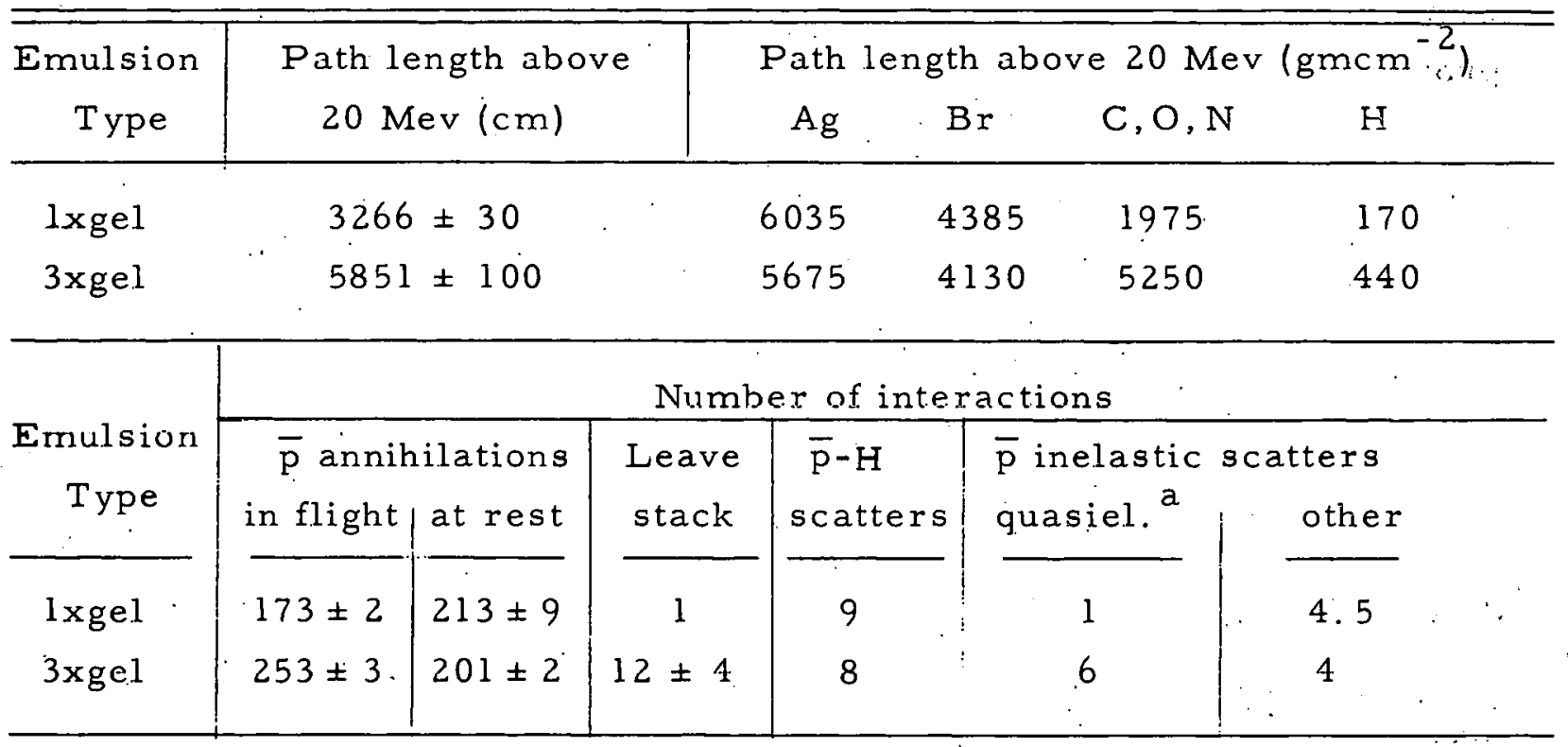

a. By quasielastic events we:mean those that $c$ annot be distinguished on. visual inspection alone from $\bar{p}-p$ elastic scatters. 


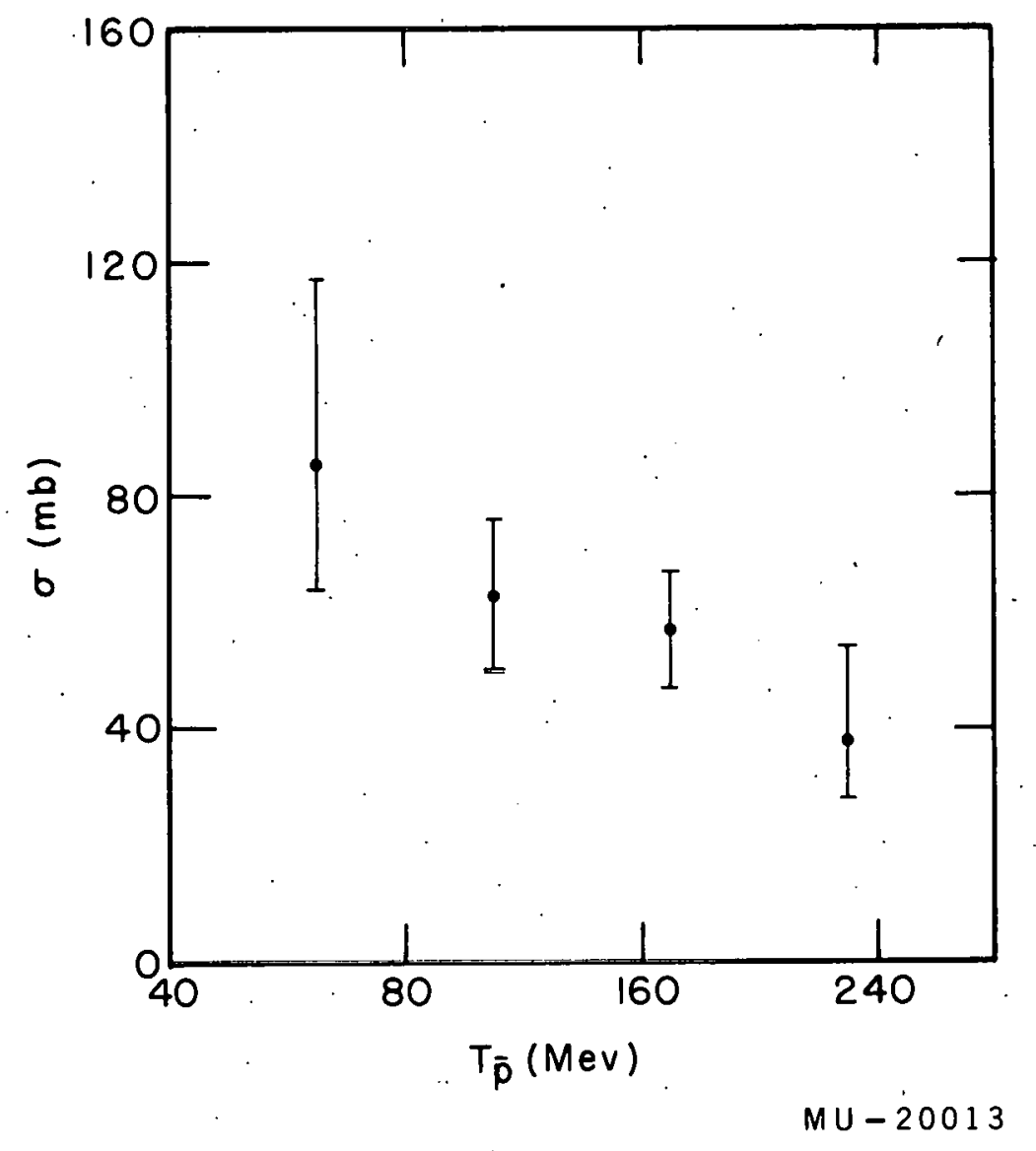

Fig. 12. The $\bar{p}-\mathrm{p}$ elastic scattering cross section for the combined emulsion and propane bubble chamber data at low energy. 


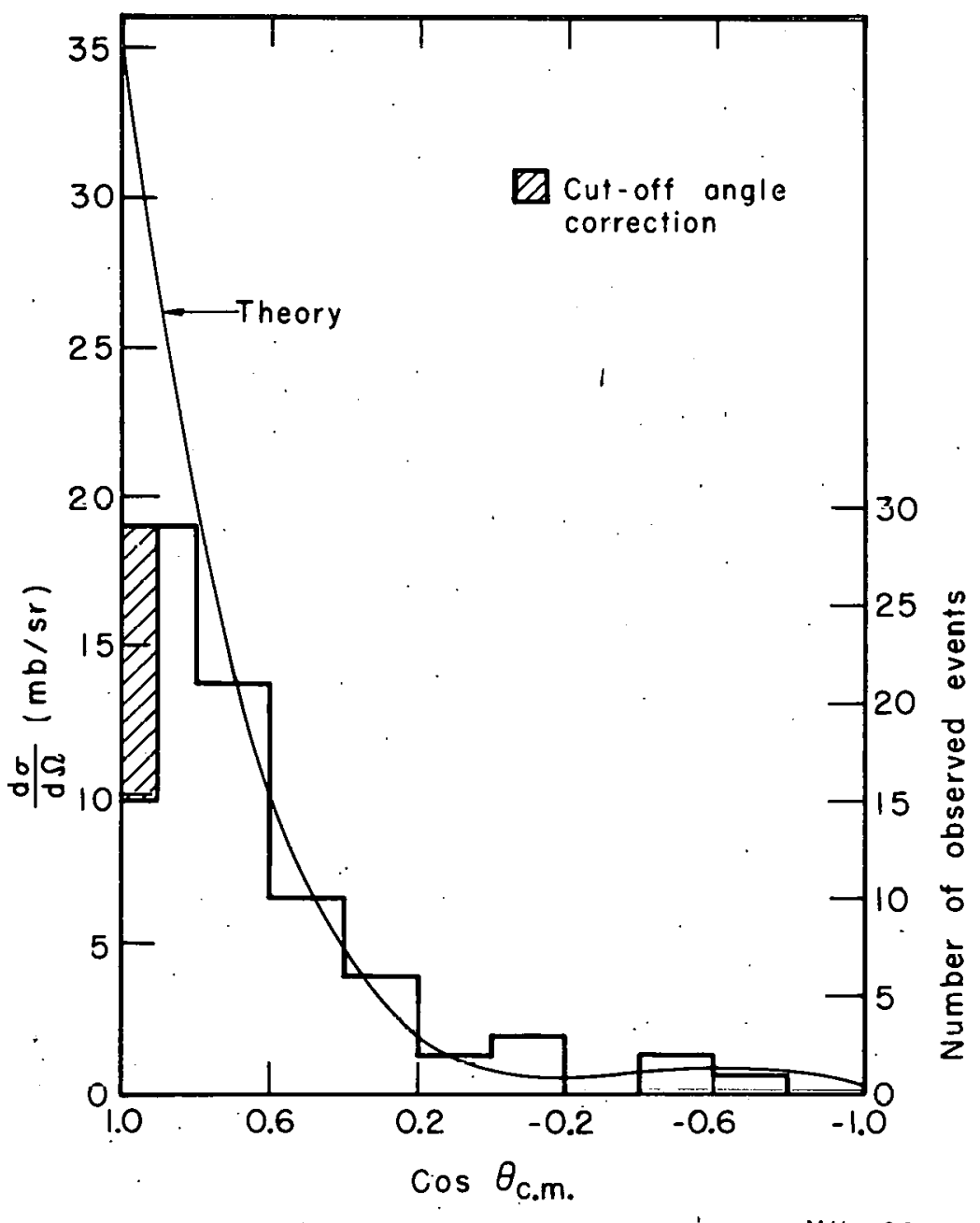

Fig. 13. The differential cross section of $\vec{p}=p$ elastic scattering for the combined emulsion and propane bubble chamber data at $75 \leq \mathrm{T}(\overline{\mathrm{p}}) \leq 200 \mathrm{Mev}$. 


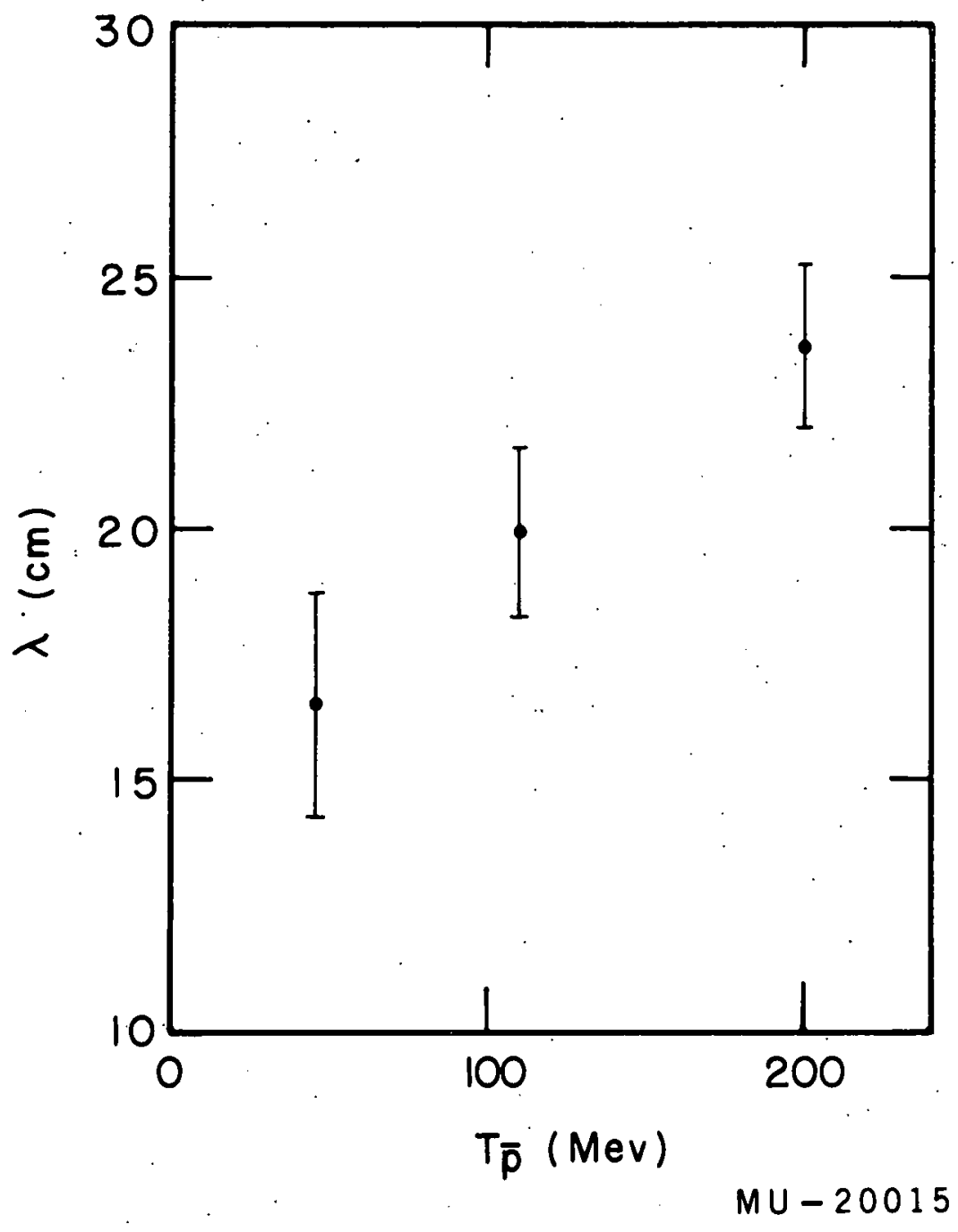

Fig. 14. The average annihilation mean free path of $\bar{p}$ in lxgel and $3 \times$ gel emulsions, weighted according to the respective path lengths. 
The above equations yield $\sigma_{h}{ }^{a}=1720 \pm 175 \mathrm{mb}$ and $\sigma_{\ell}{ }^{a}=545 \pm 105 \mathrm{mb}$ as the average antiproton annihilation cross sections in silver bromide and in gelatin with hydrogen excluded, respectively, at an average antiproton energy of $150 \mathrm{Mev}$.

With reference to a typical "geometric" cross section $\sigma_{0}=\pi\left(1.2 \times 10^{-13} \mathrm{~A}^{1 / 3)^{2}}\right.$, we obtain $\sigma_{\ell}^{a}=2.15 \pm 0.4 \sigma_{0}$ and $\sigma_{h}^{a}=1.85 \pm 0.2 \sigma_{0}$.

Again omitting the antiproton-hydrogen annihilation stars, we obtain from the previous equations $30( \pm 6) \%$ for the fraction of anrihilations with light nuclei in lxgel emulsions and $70( \pm 6) \%$ with the heavy nuclei. In 3 sgel emulsions $55( \pm 6.5) \%$ annihilate in light nuclei, and $45( \pm 6.5) \%$ in the heavy nuclei.

Our data indicate that the annihilation cross section at low energy (below a $\overrightarrow{\mathrm{p}}$ kinetic energy of $40 \mathrm{Mev}$ ) may be considerably larger than at higher energies, but low statistics do not permit a definite conclusion.

\section{Annihilation in Diluted Emulsions}

1. Charged-Pion Multiplicity

We have previously discussed the annihilation process in lxgel emulsions," and shall now compare the results with those in $3 \times$ gel emulsions. Our previous analysis included only 95 stars in flight, while the present analysis includes 250 such events.

Fìgure 15 gives the observed charged-pion multiplicity distribution. Events.closer than $25 \mu$ to the surface of the emulsion have been excluded. No corrections for pion absorbtion or detection efficiency have been included in this graph. The average number of charged pions directly observed, $\left\langle N_{\pi^{ \pm}}{ }^{ \pm}=2.31 \pm 0.16\right.$, is to be compared with the value $\left\langle N_{\pi^{ \pm}}\right\rangle=2.30 \pm 0.28$ in lxgel emulsions.

Because of less absorption, the value in $3 x g e l$ emulsions would be expected to be higher by about 0.2 than in lxgel emulsions. The near equality of the observed.values can be explained in two ways: (a) our antiprotons in lxgel emulsions had a higher pion multiplicity than in $3 \times g e l$ emulsions because of a statistical fluctuation, and (or) (b) the efficiency of pion detection in $3 \times$ gel emulsions is less than in lxgel emulsions because of a lower relative grain density of the minimum-ionization tracks. 


\section{The Ratio of $\bar{p} p / \bar{p}$ Annihilations}

The ratio of stars with an even number of charged pions to those with an odd number depends on two factors: (a) the $p / n$ ratio at the surface of the nucleus, where the annihilation takes place, and (b) the ratio of $\bar{p} p / \bar{p}$ annihilation cross sections. If either (a) or (b) is known, we could in principle determine the other.

If we assume that the $\mathrm{p} / \mathrm{n}$ ratio is uniform throughout the nucleus, and as sume that ratio (b) is 1 , the ratios for $3 x g e l$ and lxgel emulsions will be 0.90 and 0.85 , respectively. These numbers are obtained by subtracting out the estimated number of $\overline{\mathrm{p}}-\mathrm{H}$ annihilations (see Section III-C-2). Pion absorption and detection efficiency should not change this ratio appreciably.

Our experimental results are $1.16 \pm 0.15$ in $3 x g e l$ and $0.72 \pm 0.14$ in lxgel for the above ratio. These results are not inconsistent with the above assumptions.

\section{The Pion Energy Spectrum}

Figure 16 gives the energy spectrum of pions for a kinetic energy below $90 \mathrm{Mev}$. Of all observed pions, $27.4( \pm 2.2) \%$ fall into this region. In lxgel emulsions we also obtained 27.4\%. Neither of these values is:corrected for detection efficiency. A determination of the pion energy spectrum at higher energies has not been attempted, since multiple scattering is reduced in $3 \times$ gel emulsions, while distortion is considerably greater.

4. The $\pi^{+} / \pi^{-}$Ratio at Low Pion Energy

Figure 16 also gives the charges of pions that come to rest. We obtain $\left(\pi^{+} / \pi^{-}\right)_{\text {obs }}=0.24 \pm 0.129^{0}$. The preponderance of the $\pi^{-}$is particularly strong up to. $60 \mathrm{Mev}$. The $\pi^{+} / \pi^{-}$ratio quoted in paper II was 0.45 , but if we consider. only events below $60 \mathrm{Mev}$, we obtain 0.25. This ratio reflects the fact, as. pointed out in II, that the $\pi^{-}$scatter more than the $\pi^{+}$, and the energy spectrum of the inelastically scattered $\pi^{-}$peaks at a lower energy than that of the $\pi^{+}$.

\section{K-Meson Multiplicity}

We have observed five $\mathrm{K}$-mesons in 250 stars, Gorrecting for absorption, the neutral modes, and those at high energy, we obtain $3.5( \pm 1.5) \%$ as the $\mathrm{K}-\mathrm{mes}$ on abundance, which is the same as in II. 


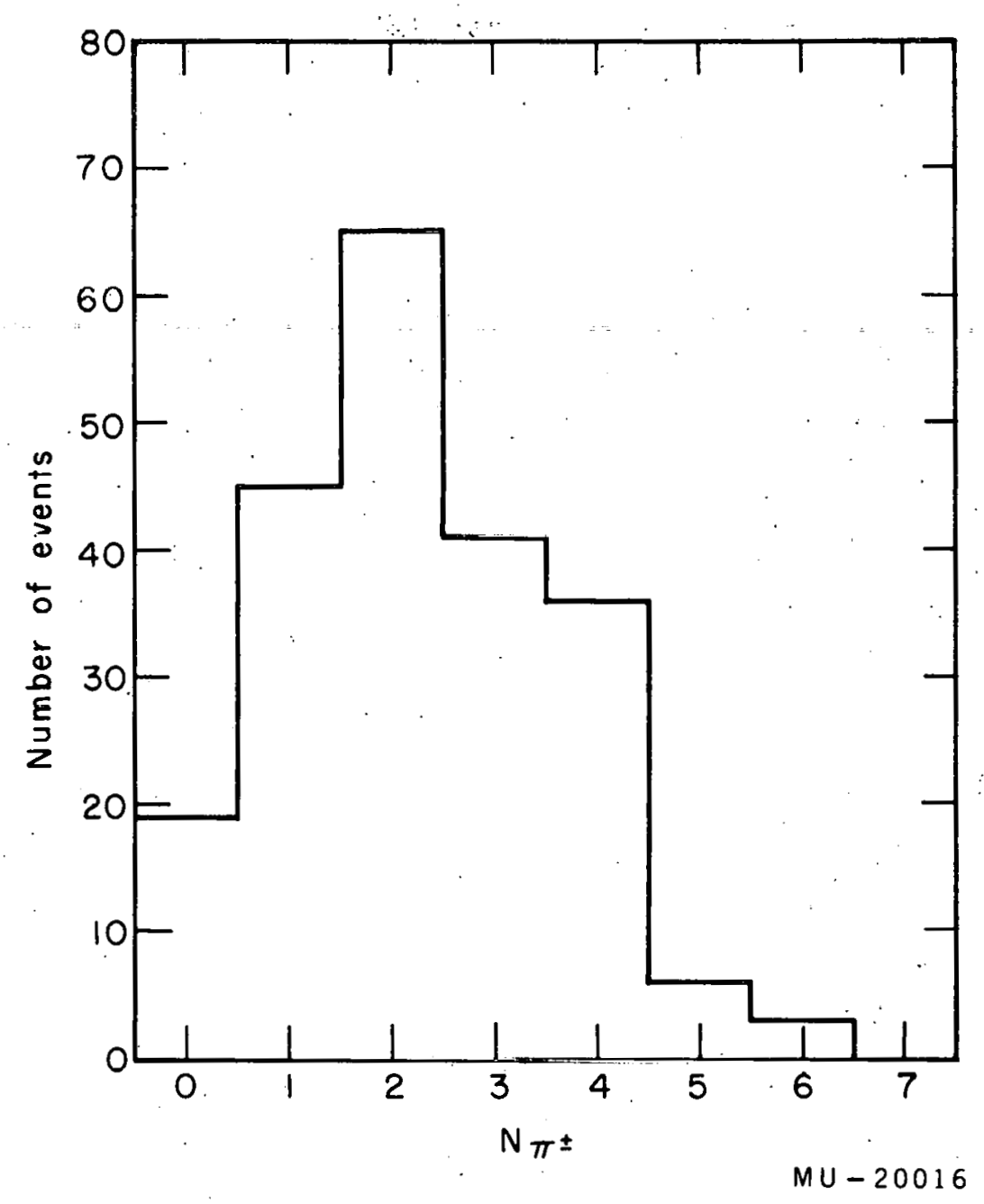

Fig. 15. The observed charged pion multiplicity spectrum in $3 x g e l$ emulsions. (No corrections for pion absorption of detection efficiency have been made here). 


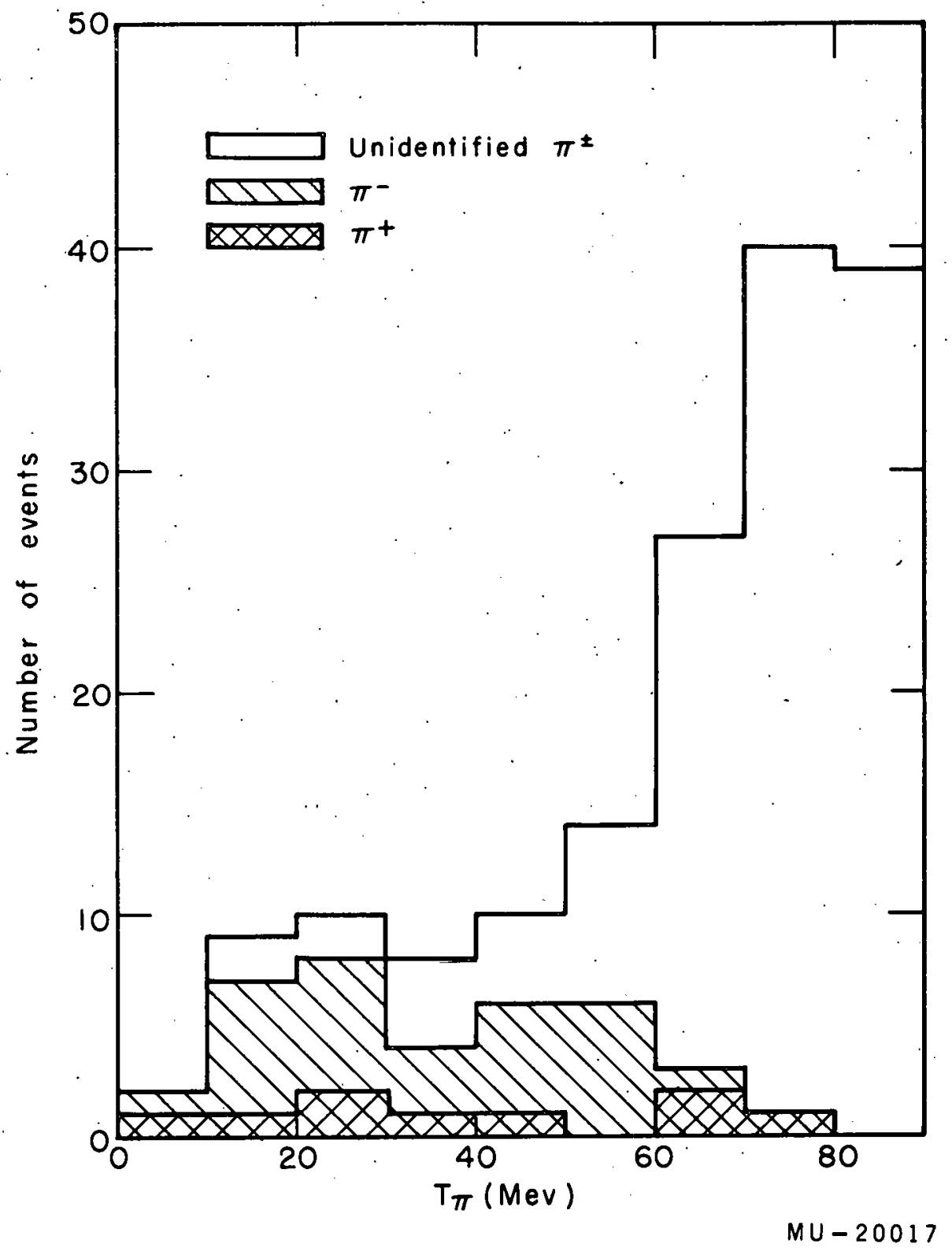

Fig. 16. The energy spectrum of low-energy pions from $\bar{p}$ annihilations in $3 \times g e l$ emulsions. 


\section{Nuclear Excitation in Diluted Emulsions}

1. General Data

Table XVI gives a summary of the data on nuclear excitation for stars in flight in 3xgel emulsions. Figure 17 gives the multiplicity distribution of heavy prongs per star. Figure 18 gives the distribution of cnergy emitted in heavy prongs per annihilation star. The energies have been assigned on the assumption that all prongs were protons.

\section{The Knock-On Process}

It is convenient to treat separately the nuclear prongs above and belów $30 \mathrm{Mev}$. Those above $30 \mathrm{Mev}$ are almost all protons, while those below, produced.after the excitation has spread to the whole nucleus, include a large fraction of alpha particles and deuterons.

As seen from Table XVI and paper II, the average energy per knock-on proton does not change noticeably with the nuclear composition. Our task now is to evaluate the number of neutrons corresponding to the observed knock-on particles in the present case. From the ratios $\langle\mathrm{n} / \mathrm{p}\rangle=1.3$ for $\mathrm{Ag}$. and $\mathrm{Br}$ and $\langle\mathrm{n} / \mathrm{p}\rangle=1.0$ for the light elements, and the fraction of annihilations with heavy and light nuclei, we evaluate the $\pi^{+} / \pi^{-}$ratio in our annihilation stars. Using the results of Metropolis et al. for nuclear cascades due to $\pi^{-}$and $\pi^{+}$ interactions, ${ }^{42}$ we obtain $1.4 \pm 0.2$ as the $\mathrm{n} / \mathrm{p}$ ratio among the knock-on particles in $3 x g e l$ emulsions. The estimated energy in both krock-on protons and neutrons is $\mathrm{U}_{\mathrm{ko}}=306 \pm 31 \mathrm{Mev}$ in $3 \times \mathrm{xgel}$ emulsions. The evaluation of $U_{k o}$ for light and heavy elements sepdralely is Hul with while yct bccaues of the large statistical error.

Figure 19 gives the kinetic energy spectrum of the knock-on prongs. The high-energy knock-on protons (those with $\mathrm{T}_{\mathrm{p}} \geq 100 \mathrm{Mev}$ ) exhibit a strong forward peaking with respect to the direction of the incident antiproton. The ratio of those in the forward hemisphere to those in the backward is $3.0 \pm 0.7$. This is considerably larger than the forward peaking expected for pions in the lab system for an isotropic distribution in the c.m. system. The latter distribution is shown in Fig. 4 inill. The forward peaking of the high-energy protons provides further evidence that the annihilation takes place at the surface of the nucleus, and that hence most of the pions absorbed are those going for ward.with respect to the direction of the incoming antiprotons. Figure 20 gives the angular distribution of knock-on protons for $\mathrm{T}_{\mathrm{p}} \geq 100 \mathrm{Mev}$. 
Table XVI

The average values for the number of heavy prongs, the energy per heavy prong, and the energy of heavy prongs per star, for $\bar{p}$ interactions in flight in emulsions. a

\begin{tabular}{|c|c|c|c|c|c|c|c|c|c|c|c|}
\hline \multirow[b]{3}{*}{ Emuls. } & \multirow[b]{3}{*}{$\mathrm{N}_{\pi} \pm$} & \multirow{3}{*}{$\begin{array}{l}\text { No. } \\
\text { of } \\
\text { stars }\end{array}$} & \multirow{2}{*}{\multicolumn{3}{|c|}{ No. of prongs }} & \multicolumn{5}{|c|}{ Energy (Mev) } & \multirow[b]{3}{*}{$\left\langle\Sigma E_{H}\right\rangle$} \\
\hline & & & & & & & er prong & & & star & \\
\hline & & & $\left\langle\mathrm{N}_{\mathrm{ev}}\right\rangle$ & $\left\langle N_{k o}\right\rangle$ & $\left\langle\mathrm{N}_{\mathrm{H}}\right\rangle$ & $\left\langle E_{e v}\right\rangle^{c}$ & $\left\langle E_{k 0}\right\rangle$ & $\left\langle E_{H}\right\rangle$ & $\left\langle\Sigma \mathrm{E}_{\mathrm{e} v}\right\rangle$ & $\left\langle\Sigma E_{k 0}\right\rangle$ & \\
\hline $3 \times g e l$ & $0.2^{d}$ & 119 & 3.3 & 1.4 & 4.7 & 16.4 & 110.8 & 44.3 & 54.1 & 153.0 & 207.1 \\
\hline $3 \times$ gel & 3 & 41 & 3.0 & 1.2 & 4.2 & 16.7 & 97.7 & 39.4 & 50.3 & 116.2 & 166.5 \\
\hline $3 x g e l$ & $4-6$ & 45 & 2.2 & 0.8 & 3.0 & 17.2 & 87.0 & 35.6 & 39.5 & 67.1 & 106.6 \\
\hline $3 \times g e 1$ & $0-6 \mathrm{e}$ & 243 & 3.07 & 1.23 & 4.30 & 16. 7 & 103.7 & 41.5 & 51.2 & 127.6 & 178.8 \\
\hline lxgel & $0-6^{\mathrm{f}}$. & 95 & 3.55 & 1.54 & 5.09 & 18.0 & 101.8 & 43.3 & 63.9 & 156.4 & 220.3 \\
\hline
\end{tabular}

a. The corresponding table for lxgel emuisions is given in II. Note that $\left(E_{F}\right.$ ) does not depend much on the emulsion composition.

These energies include a binding energy of $8 \mathrm{Mev}$ per prong.

c. The energies were assigned on the assumption that all prongs were protors. Actually deuterons and alpha particles are also present, and a correction for this effect is made later.

d. No $\bar{p}$ disappearances have been included, nor have stars with $N_{\pi^{ \pm}}=0$ and small visible nuclear energy release.

e. These events include events occurring near the surface of the emulsion $(\Delta z<25 \mu$ ), for which no pion multiplicity was assigned.

f. From pape $=$ II, Table V. 


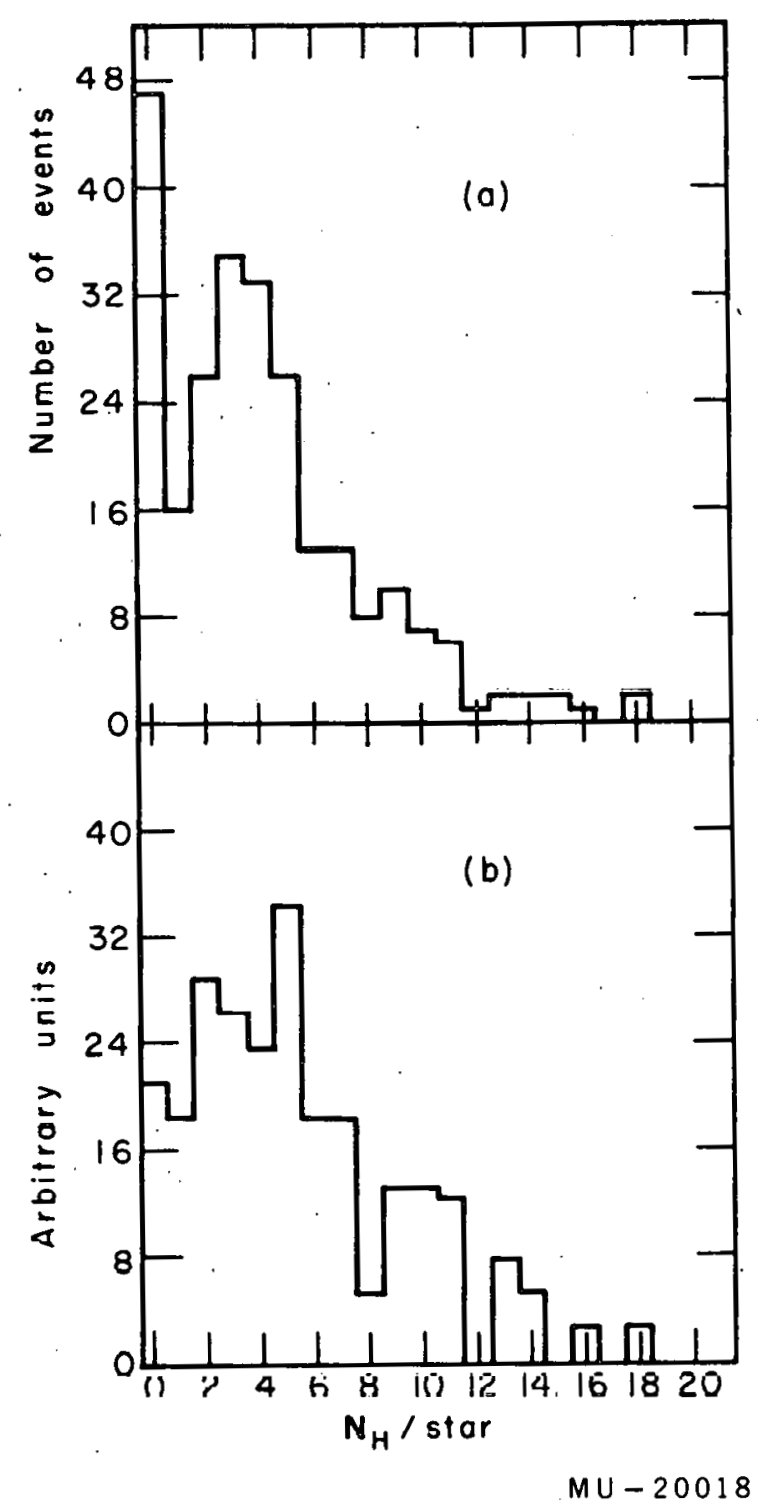

Fig. 17. The multiplicity distribution of heavy prongs per star in flight for (a) 3xgel emulsions and (b) lxgel emulsions. (The number of events is normalized so as to be equal to that in $3 \times g e l .1$ 


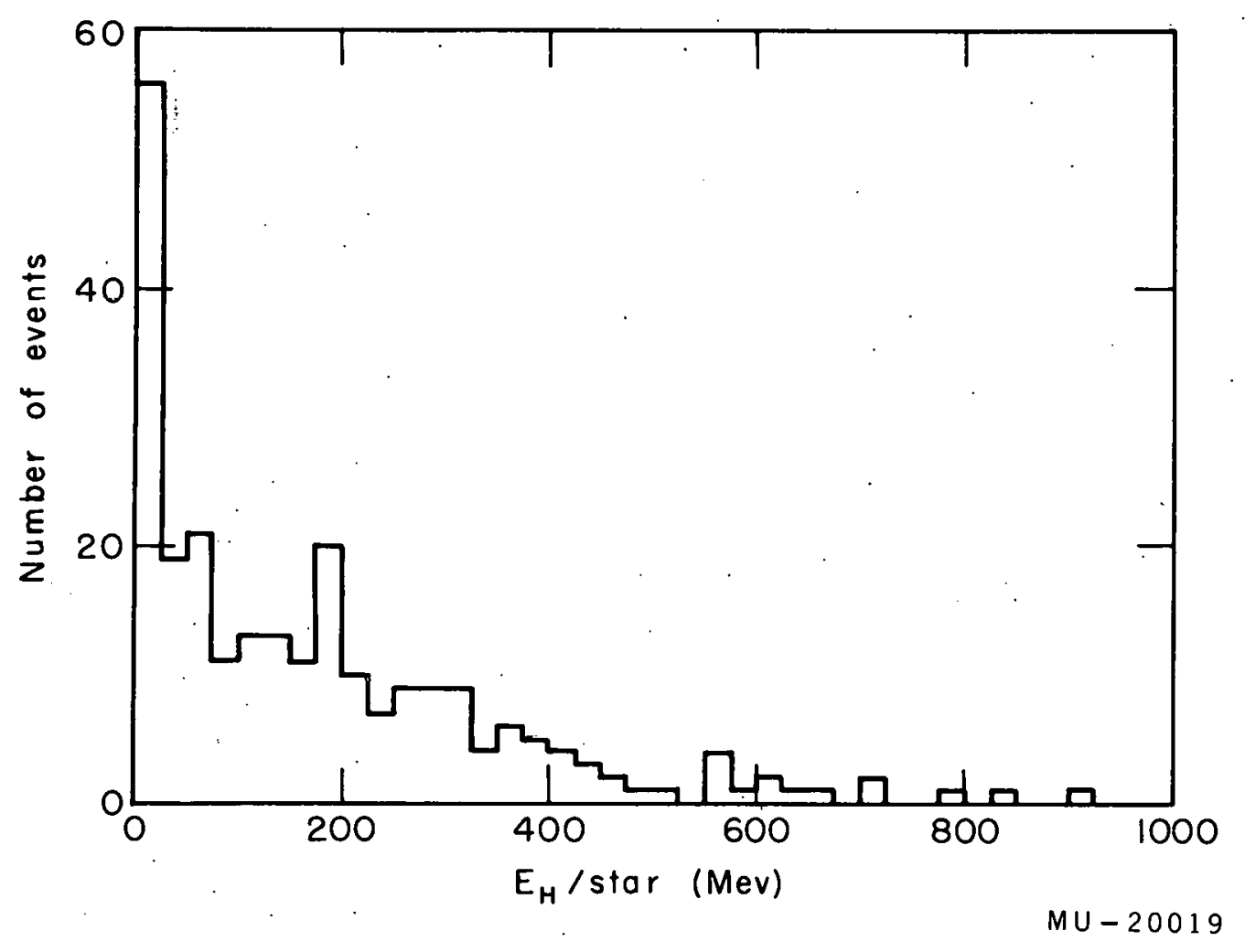

Fig. 18. The distribution of energy in heavy prongs per $\bar{p}$ annihilation star in flight, in $3 \times g e l$ emulsions. It has been assumed that all heavy prongs are protons, although deuterons and alpha particles are present. 


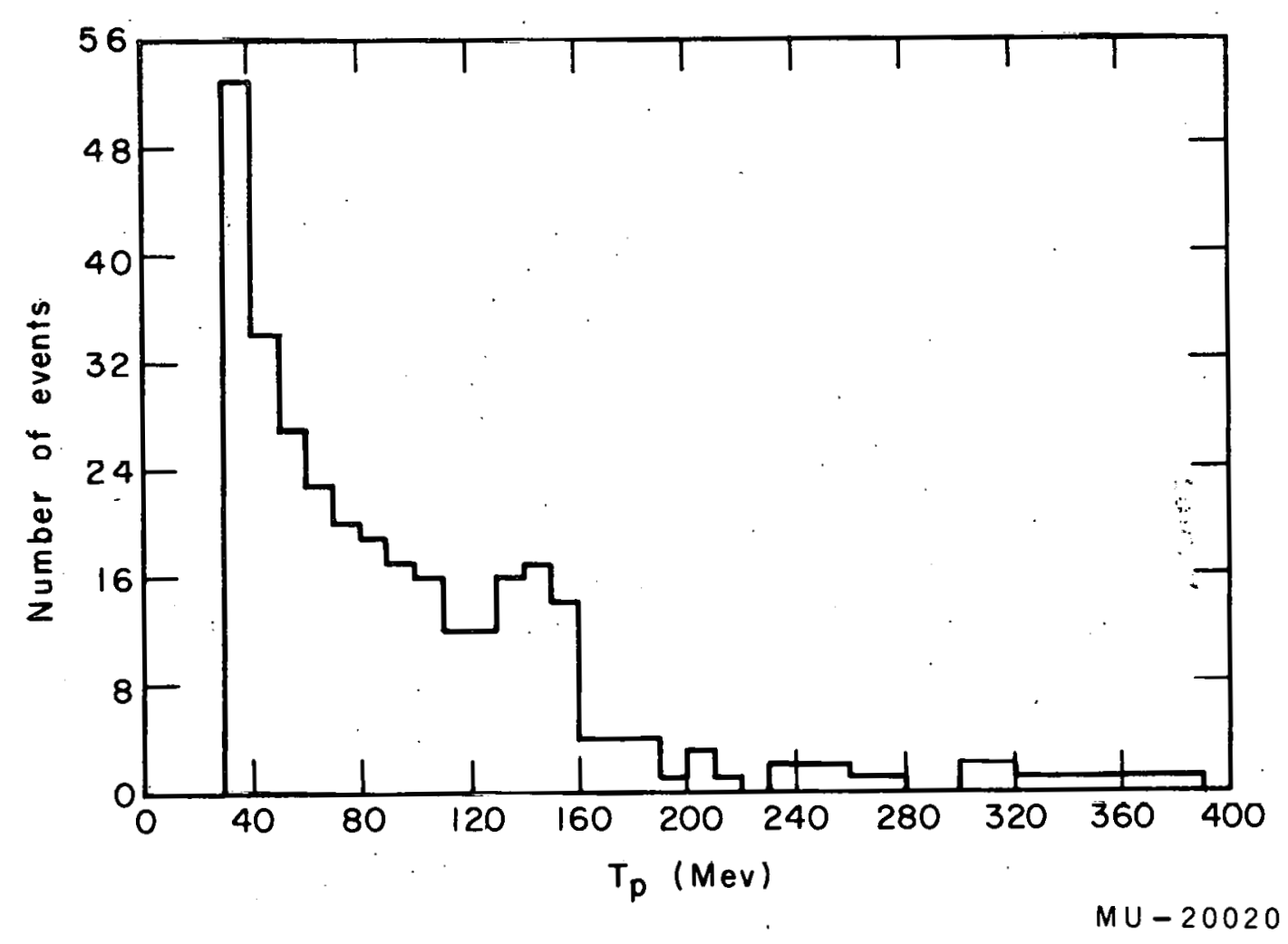

Fig. 19. The kinetic-energy spectrum of knock-on prongs in $3 \times g e l$ errulsiuns $\Gamma_{1}$ un $\bar{p}$ aninililation stara in flight. 


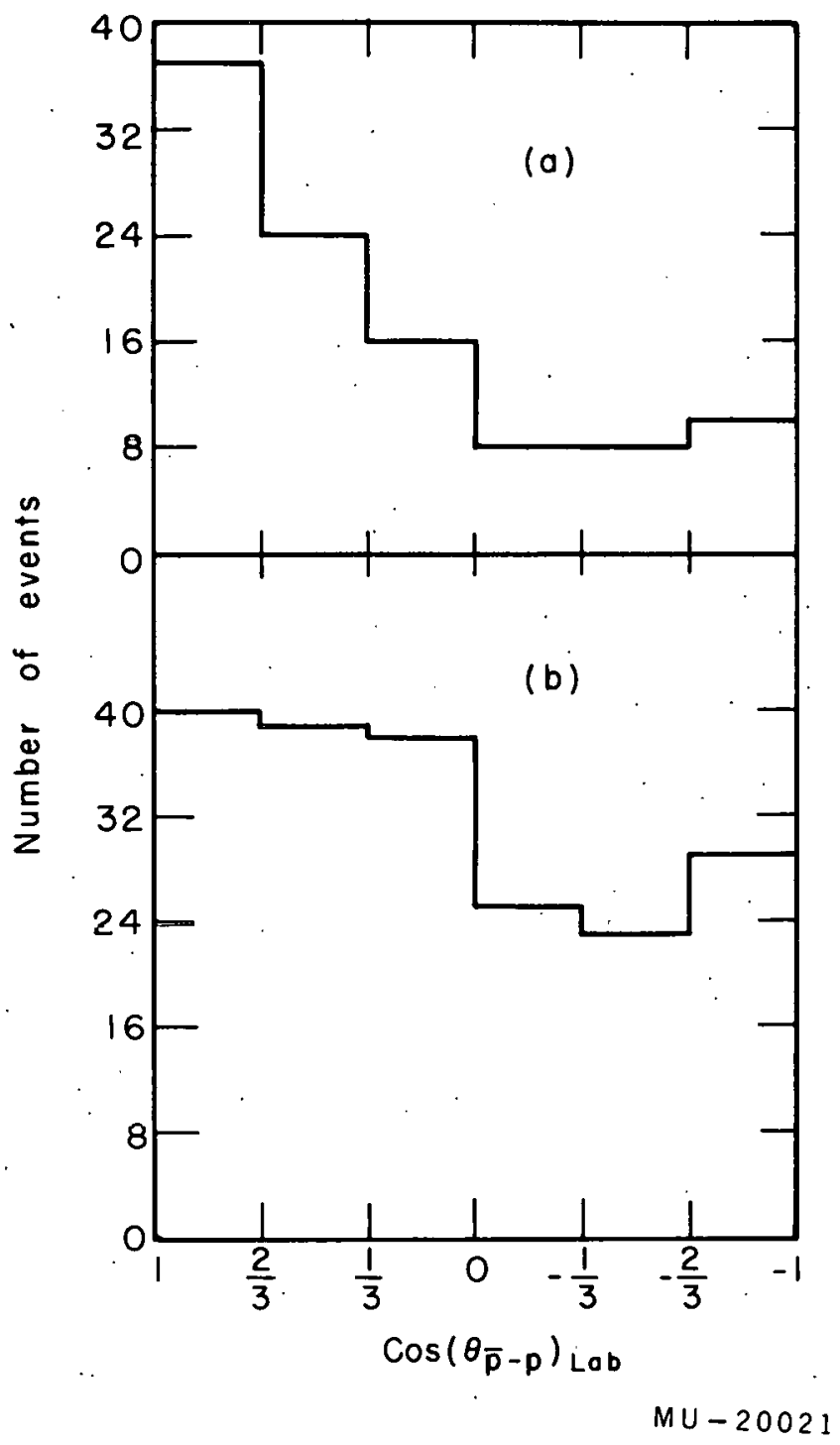

Fig. 20. The angular distribution of knock-on protons with respect to the incoming antiproton for (a) $T_{p} \geq 100 \mathrm{Mev}$ and (b) $100>\mathrm{T}_{\mathrm{p}} \geq 30 \mathrm{Mev}$ 。 
For knock-on protons in the energy range $100>\mathrm{T}_{\mathrm{p}}>30 \mathrm{Mev}$, the forward peaking is much less pronounced. The forward-to-backward ratio of protons is $1.5 \pm 0.2$ (see Fig. 20). Part of these protons are due to nuclear collisions of the primary knock-on nucleons. They represent an intermediate stage between the knock-on and the evaporation process, where the energy has been distributed among all the nucleons, and the direction of proton emission has become isotropic. Another reason for the reduced forward peaking is the following: Since annihilations take place at the surface of the nucleus, almost all of the absorbed pions are going in the forward direction with respect to the antiproton. Using the two-nucleon model of pion absorption, we see that the nucleons that in addition would carry off the momentum of the pions would be those in the forward hemisphere. Those in the backward hemisphere would be expected to be slower.

\section{The Evaporation Process}

Evaporation theory is used to describe the fate of highly excited nuclei. The theory is less applicable to light nuclei, which tend to disintegrate completely when highly excited. A rccent comprehensive treatment of evaporation has been published by Dostrovsky, Rabinowitz, and Bivins, who used Monte Carlo calculations. 43 They show that at a given nuclear temperature, the average number of nucleons lost is proportional to the atomic weight of the initial nucleus, and that the average energy per evaporated nucleon at a given nuclear temperature is independent of the mass of the nucleus.

The nuclear exeitation wao cotimatod from the average number uf rviliorated charged particles per nonhydrogen nucleus, with the nuclear temperature assumed to be the same for the light and heavy nuclei. The average number of evaporated charged particles was evaluated as a function of the nuclear temperature by using the graphs of Dostrovsky et al. for silver and extrapolating for bromine. 43 For the light nuclei, we have assumed that the ratio of neutrons to charged particles (protons, alpha particles, deuterons, and various fragments) is 0.4 among the evaporated particles. The method of evaluating this number is similar to that of Menon et al. ${ }^{44}$ By weighting the number of charged particles evaporated from various species of nuclei at a certain nuclear temperature by the probability of annihilation with those nuclei, the number of charged particles evaporated was derived as a function of the nuclear temperature for $3 x g e l$ emulsions. A comparison with the experimental evaporation- 
prong multiplicity led to a nuclear temperature of $5.3 \mathrm{Mev}$. The initial excitation of nonhydrogen nuclei was thus found to be $143 \pm 25 \mathrm{Mev}$.

An independent estimate on the nuclear temperature was obtained from the slope of the curve in Fig. 21, which gives the number of protons per energy interval as a function of the proton kinetic energy. We obtained a nuclear temperature of $5.1 \pm 1.0 \mathrm{Mev}$, which is in good agreement with the above estimate as well as with a similar calculation on nuclear excitation in the $\bar{p}$ annihilation process in lxgel emulsions.

The fraction of alpha particles emitted from light nuclei, which often are completely broken up by nuclear excitation, should be considerable higher than from heavy nuclei. The Coulomb barrier should prohibit the evaporation of protons with a kinetic energy of less than about $3 \mathrm{Mev}$. We shall assume that the particles with a range of $50 \mu$ or less in lxgel emulsions (corresponding to an alpha-particle range of $9 \mathrm{Mev}$ ) and a range greater than nuclear recoils ( $5 \mu$ or less) are low-energy alpha-particles. 44 We then find that $8.9 \%$ of the heavy prongs in lxgel emulsions and $13.4 \%$ in the $3 x g e l$ emulsions are such low-energy evaporated alpha particles. The difference becomes even more pronounced if we treat separately the annihilation events with one to five heavy prongs--primarily from light nuclei--and those with six or more-almost all of which come from the heavy nuclei. In $3 x g e l$ emulsions we find experimentally $19.8( \pm 2.4) \%$ and $8.9( \pm 1.3) \%$ slow alpha particles from stars with one to five and six or more heavy prongs respectively.

The average number of heavy prongs emitted from heavy nuclei and light nuclei in emulsions is about seven and three, respectively, for $\bar{p}$ annihilations in flight at an average energy of $150 \mathrm{Mev}$.

\section{Total Nuclear Excitation and Pion Absorption}

For annihilations in flight, at an average energy of $150 \mathrm{Mev}$, in $3 \times g e l$ emulsions, the average energy. per star was found to be $442 \pm 40 \mathrm{Mev}$. From the data of Appendix $A$ we estimate that $1.1 \pm 0.2$ pions are absorbed per annihilation. On the basis of Appendix B we conclude that the number of charged pions absorbed is $0.8 \pm 0.2$. 


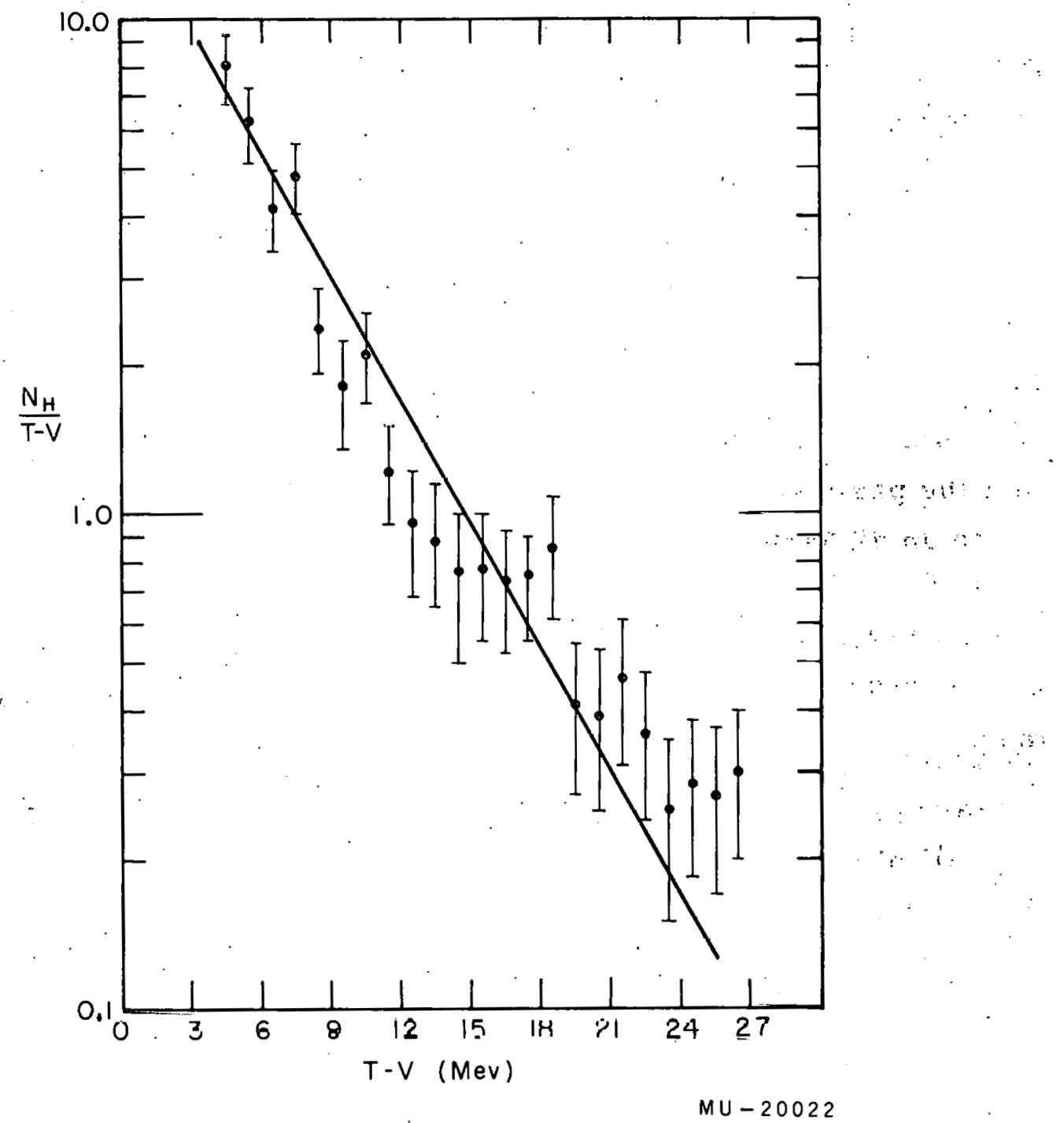

Fig. 21. The number of evaporation protons in $3 \times g e l$ emulsions per unit energy interval (defined as $\mathrm{N}_{\mathrm{H}}$ in this figure) from $\bar{p}$ annihilations in flight. The Coulomb barrier $\mathrm{V}$ has been chosen here as $3 \mathrm{Mev}$. 


\section{ACKNOWLEDGMENTS}

I would like to express appreciation to Professor Gerson Goldhaber for his advice and encouragement during this work and through several years of graduate study:.

I am grateful to Dr. Sulamith Goldhaber for guidance and encouragement on the analysis of the propane bubble chamber experiment.

For the beam setup for the emulsion exposure I am indebted to $\because \cdots$. Drs. Emilio Segrè, Edward Lofgren, Louis Agnew, Jr., Clyde Wiegand, Thomas Ypsilantis, and Herbert Steiner and Mr. Tom Elioff; and for the bubble chamber exposure to Drs. Luis Alverez, Harold Ticho, Phillippe Eberhard, and Myron Good.

The 30-in. propane bubble chamber was built and operated under the direction of Professor Wilson Powell, and the IBM programming for the analysis was possible through the assistance of $\mathrm{Mr}$. Howard White.

Many thanks for advice are due Drs. William Fowler, Tsang Fung Hoang, and Theodore Kalogeropoulos.

Thanks are due to $\mathrm{Mr}$. Wonyong Lee, Mr. Thomas O'Halloran, and Mr. Theodore Stubbs as co-workers on some of the projects. The author is indebted to Mr. Kirmach Natani, Mr. Yun-Tong Fung, Mr. Michael Guinan, and Mrs. Marjory Simmons for miscellaneous assistance.

I would like to express my thanks for scanning and for some calculations to Mr. Bruce Boyd, Miss Joan Dyleski, Mrs. Jean Firby, Mr. James Glass, Mrs. Frances Glenn, Mrs. Lora Langner, Mrs. Rosanne Levitt, Miss Harriett Rice, Mrs. Evelyn.Rorem, Mrs. Elisabeth Russell, Mrs. Mary-Lou Santos, Miss Charlotte Scales, Mrs. Louise Shaw, and Mrs. Frances Simon.

This work was done under the auspices of the U. S. Atomic Energy Commission. 


\section{APPENDICES}

\section{A. Absorption and Scattering of Pions}

The number of pions absorbed in propane and in $3 \times$ gel has been evaluated from (a) the energy in nuclear excitation, (b) the average energy per absorbed pion, and (c) the fraction of inelastically interacting pions that are scattered. The latter two quantities are evaluated in this Appendix.

Almost all the energy given to nucleons in the annihilation process comes from absorption or inelastic scattering of the annihilation products by the nucleons. The inelastic scattering of pions also reduces the average energy of the pions, modifying the pion energy spectrum. For these reasons, it is useful to study the inelastic pion scattering before attempting to find the number of interacting pions and the nuclear excitation.

Appendix VI in II gives a summary of the data compiled from various authors on inelastic scattering of pions in emulsion nuclei, on which the calculations in II were based. In the energy interval from 100 to $200 \mathrm{Mev}$, the fraction of nonelastically interacting $\pi^{-}$that give rise to inelastic scattering is $0.27 \pm 0.04$, and for $\pi^{+}$the corresponding value is $0.20 \pm 0.04$. Of the interacting pions, the $\pi^{-}$are morellikely to undergo inelastic scattering.

Figure 22 gives the average energy of pions after inelastic scattering in emulsion nuclei as a function of the initial pion energy. This figure is based on the data of Appendix VI in II and the more recent values of Belovitskii at $300 \mathrm{Mev} .{ }^{45}$ We notice that the negatively charged pions undergo greater energy losses than the positively charged ones. 'l'he best-fit curves are also drawn in.

The energy spectrum of the inelastically scattered pions can be empirically fitted by a function resembling the Maxwellian distribution function with a cut-off at the high e nergy end.

The above data, together with the pion absorption and scattering mean free path in nuclear matter, as given by Frank, Garminel, and Watson (hereafter called FGW), ${ }^{46}$ were used to evaluate the primary pion energy and multiplicity in II.

Recent publications of experimental work $45,47-49$ show that the pionabsorption cross section decreases rapidly after about 200-Mev pion energy, which was not expected from an extrapolation of the FGW theory to high pion energies. Beyond $350 \mathrm{Mev}$, inelastic scattering starts to predominate over pion absorption. 


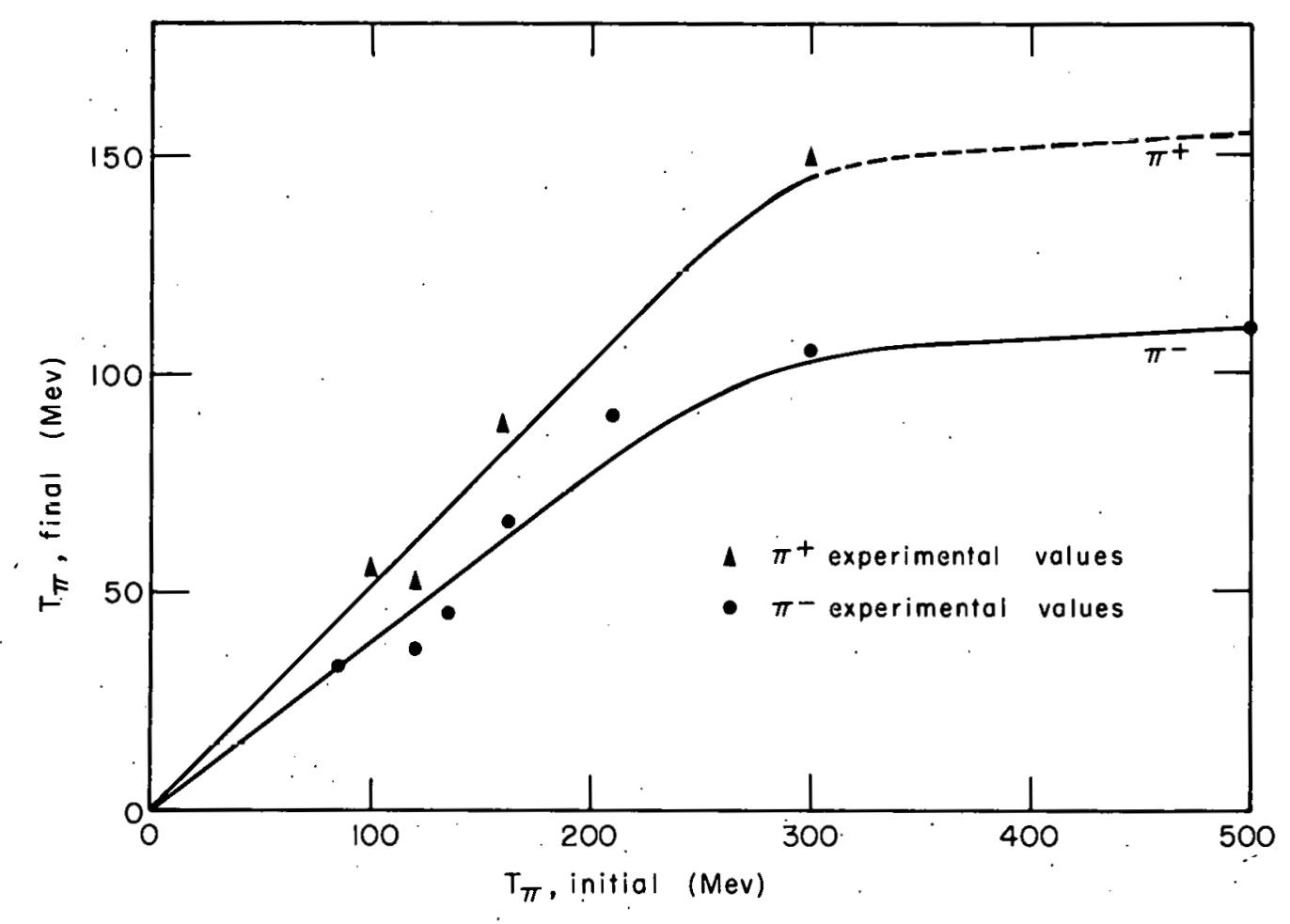

$M U-20023$

Fig. 22. Compiled data on the average energy of pions after inelastic scattering in lxgel emulsions. 


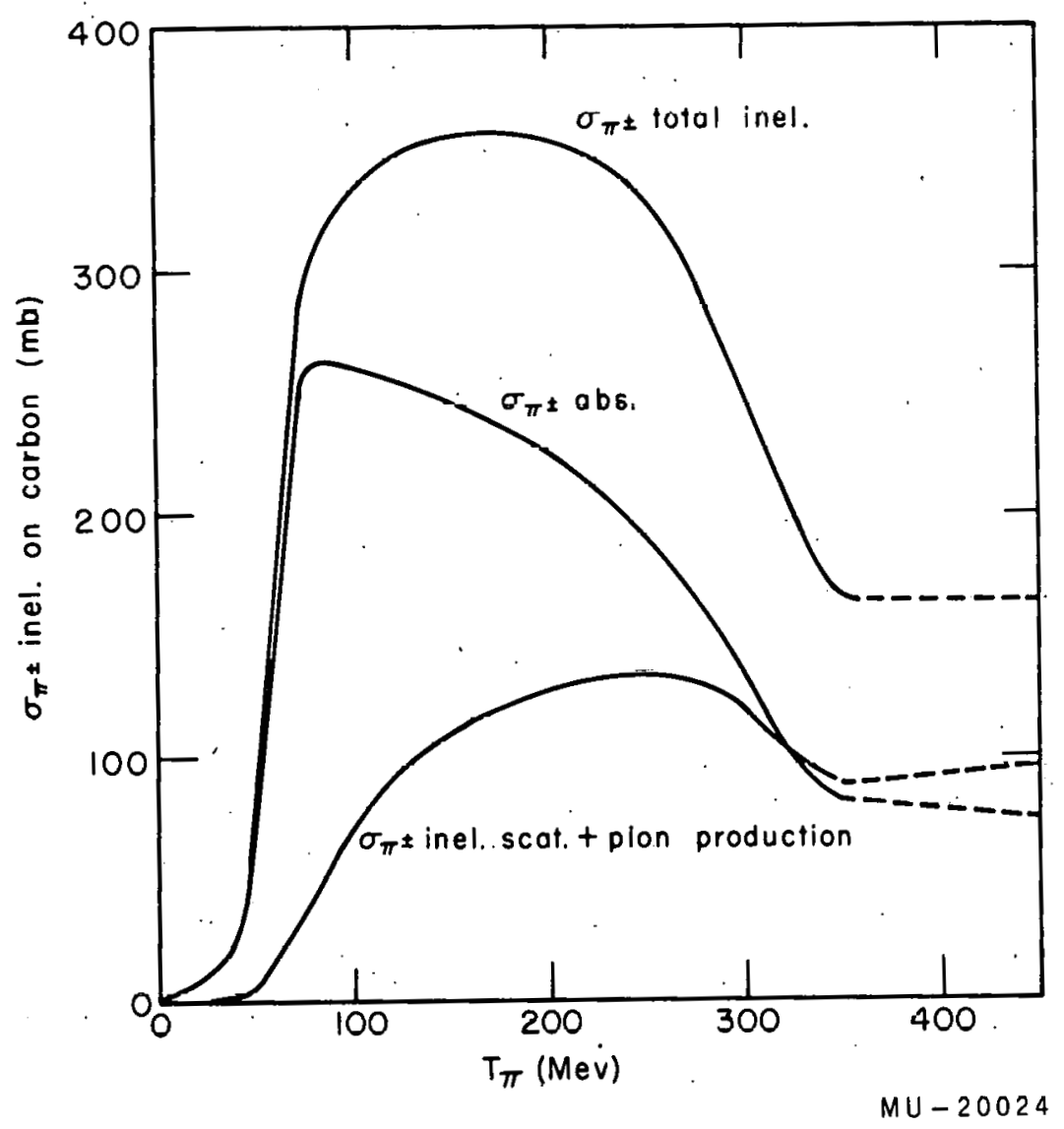

Fig. 23. The compiled data on the absorption and scattering cross sections for $\pi^{ \pm}$in carbon. 
Experiments with light and heavy nuclei indicate that the fraction of inelastically interacting pions absorbed is slightly higher in heavy than in light elements. 50,51 In heavy elements (with a neutron excess) the $\pi^{-}$scattering cross section is larger than for $\pi^{+}$. We estimate that for $T_{\pi} \geq 125 \mathrm{Mev}$ the fraction of nonelestically interacting $\pi^{-}$that are scattered in lxgel emulsions is about 0.95 of that in carbon. For $\pi^{+}$the corresponding ratio is 0.8 . Using these estimates and the data from all the above references as well as the lowenergy data of Bernardini and Levy, ${ }^{52}$ we construct Fig. 23. This figure gives the $\pi^{ \pm}$absorption and inelastic scattering cross sections in carbon as a function of the pion kinetic energy. The data on absorption and scattering cross sections beyond $350 \mathrm{Mev}$ are still very scanty, but no rapid changes are indicated. $53,49$. For simplicity we shall assume the total inelastic cross section in this region to be constant. This simplification is also justified in our calculations by the fact that only a small fraction of the pions we consider are in that high-energy region.

By weighting over the pion energy spectrum for $\left\langle N_{\pi}\right\rangle=5.0$ for $\bar{p}$ annihilations at rest and taking into account the $\pi^{-} / \pi^{+}$ratio on annihilation, we find that of the nonelastically interacting pions, $68 \%$ undergo absorption and $32 \%$. inelastic scattering in lxgel emulsions. The average kinetic energy of the absorbed pions is $202 \mathrm{Mev}$, and of those inelastically sçattered, $251 \mathrm{Mev}$.

Using the pion energy spectrum of the hydrogen-like events with $4 \pi^{ \pm}$ for $\overline{\mathrm{p}}$ annihilation at $1050 \mathrm{Mev} / \mathrm{c}$, we find that of the nonelastically interacting pions $60.5 \%$ are absorbed and $39.5 \%$ inelastically scattered in carbon. The average energy for the absorbed pions is $245 \mathrm{Mev}$ and $341 \mathrm{Mev}$ for those inelastically scattered.

The published data 50,51 on the final energies of pions inelastically scattered in carbon nuclei indicate that they are the same or about $5 \mathrm{Mev}$ higher than of those scattered in emulsion nuclei for $T_{\pi}$ initial $\geq 100 \mathrm{Mev}$.

For emulsions and for such antiproton energies as in ACE and II, the evaluation of the number of pions absorbed, based on this Appendix, leads to closely similar values as obtained in ACE and II. 


\section{B. Neutral Pion Absorption 54}

For the evaluation of the primary $\pi^{+}, \pi^{-}$, and $\pi^{0}$ multiplicities for $\bar{p}$ annihilations in complex muclei, it is necessary to know their relative absorption cross sections. Some recent publications have assumed that the absorption cross section for neutral pions is half that for each of the charged pions. 42,21

Pion absorption takes place through a two-nucleon system, which we shall consider to be in the s state. We shall denote the singlet and triplet s states by the superscripts ${ }^{1} s$ and ${ }^{3} s$. Using the method of detailed balancing, we obtain the following relations between $\pi^{0}$ and $\pi^{\prime}$ absorption rates:

$a \cdot R\left(\pi^{0}+D^{3} s \rightarrow n+p\right)=\frac{\sigma\left(n+p \rightarrow \pi^{0}+D^{3} s\right)}{\sigma\left(p+p \rightarrow \pi^{-1}+D^{3} s\right)} \cdot b \cdot R\left(\pi^{+}+D^{3} s \rightarrow p+p\right)$

$\left.c{ }^{0}+\left(\pi^{0}+p\right)^{1} s \rightarrow n+p\right)=\frac{\sigma\left(n+p \rightarrow \pi^{0}+(n+p)^{1}\right)^{1}}{\sigma\left(p+p \rightarrow \pi^{+}+(n+p)^{s}\right)} \cdot d \cdot R\left(\pi^{+}+(n+p)^{1} s p+p\right)$

e. $R\left(\pi^{0}+(p+p)^{1} s \rightarrow p+p\right)=\frac{\sigma\left(p+p \rightarrow \pi^{0}+(p+p)^{1} s\right.}{\sigma\left(p+n \rightarrow \pi^{+}+(n+n)^{1} s\right.}$. f. $R\left(\pi^{+}+(n+n)^{1} s \rightarrow p+n\right)$

g. $R\left(\pi^{0}+(n+n)^{l} s \rightarrow n+n\right)=\frac{\sigma\left(n+n \rightarrow \pi^{0}+(n+n)^{1} s\right)}{\sigma\left(p+n \rightarrow \pi^{+}+(n+n)^{1} s\right.} \cdot h \cdot R\left(\pi^{+}+(n+n)^{1} s \rightarrow p+n\right)$

Coefficients a to $h$ represent the statistical weighting factors for the spin and isotopic spin states. We obtain $a=b=3 c=3 d=3 e=3 f=3 g=3 h$.

The cross sections for the above modes of pion production are given by $\Sigma a_{i} \sigma_{i f}$, where $i$ and $f$ represent the initial and final isotopic spin states of the nucleon-nucleon pair, respectively. The coefficient $a_{i}$ is the weighting factor for the isotopic spin states. For unlike nucleons, $a_{i}$ is one-half, because the pn pairs can be in either $I=1$ or 0 states. For like nucleons, $a_{i}$ is one, because only one isotopic spin state is initially available. The relations between these cross sections have been discussed in detail by A.H. Rosenfeld. 55 
For convenience we shall quote some of his results:

$$
\begin{aligned}
& \sigma\left(p+p \rightarrow \pi^{+}+D_{(n+p)} D^{3 s}=\sigma_{10}(D)+\sigma_{10}(n+p)+\sigma_{11}\right. \\
& \sigma\left(p+n \rightarrow \pi^{0}+\underset{(n+p)}{D^{3} s} 1 s=1 / 2 \quad\left[\sigma_{10}(D)+\sigma_{10}(n+p)+\sigma_{01}\right]\right. \\
& \sigma\left(p+p \rightarrow \pi^{0}+p+p\right)=\sigma_{11} \\
& \sigma\left(\mathrm{p}+\mathrm{n} \rightarrow \pi^{+}+\mathrm{n}+\mathrm{n}\right)=1 / 2\left[\sigma_{11}+\sigma_{01}\right] .
\end{aligned}
$$

The cross sections $\sigma_{\text {if }}$ are highly energy-dependent. Hence we have to find them at the appropriate energy, corresponding to the average energy of the absorbed $\pi$ mesons as determined in Appendix $A$. The corresponding energy for nucleon-nucleon collisions is about $700 \mathrm{Mev}$.

At a proton energy of $586 \mathrm{Mev}$, it has been determined that 56,57

$$
\sigma\left(p+p \rightarrow \pi^{0}+p+p\right)=1.6 \pm 0.2 \mathrm{mb}
$$

and

$$
\sigma\left(p+n \rightarrow \pi^{+}+n+n\right)=2.0 \pm 0.5 m b .
$$

For $970 \mathrm{Mev}$ proton energy, it was found that 56

$$
\sigma\left(p+p \rightarrow \pi^{0}+p+p\right)=5.4 \pm 1.0 \mathrm{mb}
$$

and

$$
\sigma\left(\mathrm{p}+\mathrm{n} \rightarrow \pi^{+}+\mathrm{n}+\mathrm{n}\right)=4.3 \pm 1.1 \mathrm{mb} \text {. }
$$

On-this basis we assume that, at $700 \mathrm{Mev}$,

$$
\frac{\sigma\left(p+p \rightarrow \pi^{0}+p+p\right)}{\sigma\left(p+n \rightarrow \pi^{+}+n+n\right)}=1.0 \pm 0.3
$$

and

$$
\sigma_{11} \approx \sigma_{01}
$$


Using this information and the relations quoted from Rosenfeld, we obtain:

$$
\begin{gathered}
R\left(\pi^{0}+D^{3} s \rightarrow n+p\right)=\frac{1}{2} R\left(\pi^{+}+D^{s} \rightarrow p+p\right) \\
R\left(\pi^{0}+(n+p)^{s} \rightarrow n+p\right)=\frac{1}{2} R\left(\pi^{+}+(n+p)^{1} \rightarrow p+p\right) \\
R .\left(\pi^{0}+(n+p)^{s} \rightarrow p+p\right)+R\left(\pi^{0}+(n+n)^{s} \rightarrow n+n\right)=(2.0 \pm 0.6) R\left(\pi^{+}+(n+n)^{1} \rightarrow p+n\right)
\end{gathered}
$$

The absorption cross section for neutral pions by an unlike nucleon pair is hence half that for $\pi^{+}$mesons. For like nucleon pairs, however, it is twice that for $\pi^{+}$mesons.

To determine $\sigma_{\text {abs }}\left(\pi^{0}\right) / \sigma_{\text {abs }}\left(\pi^{+}\right)$, we now have to relate by detailed balancing the reaction rates $\mathrm{R}\left(\pi^{+}+\mathrm{D}^{3} \rightarrow \mathrm{p}+\mathrm{p}\right), \quad \mathrm{R}\left(\pi^{+}+(\mathrm{n}+\mathrm{p})^{\mathrm{l}^{\prime}} \rightarrow \mathrm{p}+\mathrm{p}\right)$ and $\mathrm{R}\left(\pi^{+}+(n+n)^{\mathrm{s}} \rightarrow \mathrm{p}+\mathrm{n}\right)$, using the cross sections for the inversereactions. From the work of Batson et al. ${ }^{58}$ we estimate that, at $T_{p}=700 \mathrm{Mev}$, $\sigma\left(p+p \rightarrow \pi^{+}+n+p\right)=8 \cdot m b, \cdot \sigma\left(p+p \rightarrow \pi^{+}+D\right)=2 m b$, and $\sigma\left(p+p \rightarrow \pi^{0}+p+p\right) \approx \sigma\left(p+n \rightarrow \pi^{+}+n+n\right) \approx 3.5 \cdot m b$.

We thus express all the absorption rates in terms of a single absorption rate. By taking the sum of the $\pi^{0}$ absorption rates and weighting them according to the number of states, and treating the $\pi^{+}$absorption rates in the same way, we obtain $\sigma_{\text {abs }}\left(\pi^{0}\right) / \sigma_{\text {abs }}\left(\pi^{+}\right)=0.9 \pm 0.2$ 


\section{FOOTNOTES}

1. O. Chamberlàin, E. Segrè,C. Wiegand, and T. Ypsilantis, Phys. Rev. 100, 947 (1955).

2. O. Chamberlain, W.W. Chupp, A.G. Ekspong, G. Goldhaber,

S. Goldhaber, E.J. Lofgren, E. Segrè, C. Wiegand, E. Amaldi,

G. Baroni, C. Castagnoli, C. Franzinetti,, and.A. Manfredini, Phys.

Rev. 102, 921 (1956).

3. J.S. Ball and G.F. Chew, Phys. Rev. 109,1385 (1958).

1. J.R. Fulco, Phys. Rev. 110,784 (1958).

5. J.S. Ball and J.R. Fulco, Phys. Rev. 113, 647 (19.59).

6. A.E. Glassgold, Phys. Rev. 110,220 (1958).

7. G. Goldhaber, N.B. Fowler, S. Goldhaber, T.F. Hoang, T.E.

Kalogeropoulos, and W. M. Powell, Phys. Rev. Letters 3, 181 (1959).

8. E. Fermi, Progr. Theoret. Phys. (Japan) 5, 570 (1950).

9. J.V. Lepore and M. Neuman, Phys. Rev. 98,1484 (1955).

10. …G. Sudarshan, Phys. Rev. 103, 777 (1956).

11. W. H. Barkas, R.W. Birge, W.W. Chupp, A. G. Ekspong, G. Goldhaber, S. Goldhaber, H.H. Heckman, D:H. Perkins, J. Sandweiss, E. Segrè̀,, F.M.Smith, D.H. Stork, L. Van Rossum, E. Amaldi, G. Baroni, C. Castagnoli, C. Franzinetti, and A. Manfredini, Phys. Rev. 105, 1037 (1957).

12. Z. Koba and G. Takeda, Progr. Theoret Phys. (Japan) 19, 268, (1958).

13. S.C. Frautschi, Progr. Theoret. Phys. (Japan) 22, 15 (1959).

14. I. Pomeranchuk; Doklady Akad: Nauk USSR 78, 889 (1951).

15. E. Eberle; Nuovo cimento 8 ; 610 (1958).

16. T. Gotô, Nuovo cimento 8,625 (1958).

17. F. Cerulus, Nuovo cimento 14, 827 (1959).

18. E. Segrè, Ann. Revs. Nuclear Sci. 8, 127 (1958).

19. O. Chamberlain, G. Goldhaber, L. Jauneau, T. Kalogeropoulos, E. Segrè, and R. Silberberg, Phys. Rev. 113, 1615 (1959).

20. L. Agnew, T. Elioff, W. B. Fowler, R. Lander, W. M. Powell, E. Segrè, H. Steiner, H. White, C. Wiegand, and T. Ypsilantis, Antiproton Interactions in Hydrogen and Carbon below $200 \mathrm{Mev}$, UCRL-8785, October 8, 1959 (unpublished). 
21. E. Amaldi, G. Baroni, G. Bellettini, C. Castagnoli, M. Ferro-Luzzi, and A. Manfredini, Nuovo cimento 14, 9.7,7 (1959).

22. P. Eberhard, M. Good, and H. Ticho, A Separated 1.17-Bey/c K Meson Beam, UCRL-8878, August 25, 1959 (unpublished).

23. Events outside the geometrical cutoffs, described in Section II-B, have been included in the present analysis.

24. C.A. Coombes, B. Cork, W. Galbraith, G.R. Lamberlson, and W. A. Wenzel, Phys. Rev. 112, 1303 (1958).

25. N. Horwitz, D. Miller, J. Murray, and R. Tripp, Phys. Rev. 115 , $472(1959)$.

26. R.C. Weingart, Antineutron Production by Charge Exchange (Thesis), UCRL-8025, October 18, 1.957 (unpublished).

27. Three events where the $\bar{n}$ annihilation took place within $1 \mathrm{~cm}$ of the $\bar{p}$ disappearance have not been included; the scattering angles in these cases were also small (less than $30 \mathrm{deg}$ in the c.m. system) but could not be accurately measured.

28. The $\bar{p}-p$ charge-exchange cross section and differential cross section is also under study by Drs. Moyer andPoirier in a hydrogen bubble chamber experiment at a $\overline{\mathrm{p}}$ momentum of $1.6 \mathrm{Bev} / \mathrm{c}$.

29. B.R. Desai, Pion Multiplicity in Nucleon-Antinucleon Annihilation, UCRL-9024, February 17, 1960 (unpublished); also private communication.

30. A. Pais, The Many..t-Meson Problem, Institute for Advanced Study, Princtulun, N.J. (impublishcd).

31. The estimated annihilations in carbon have been subtracted from the hydrogen-like events.

32. The quoted value gives the observed number of neutral pions, i.e. $\pi^{0}$ absorption has not been corrected for.

33. This value is without the projected angle cutoff.

34. Here we had to take into account the fact that stars with observed hyperons were omitted.

35. T.F. Hoang, W.B. Fowler, and W. M. Powell, Statistical-Model Analysis of $\mathrm{K}$ Meson Production by $\overline{\mathrm{p}}$ Annihilation, UCRL-8994, January 27, 1960 (unpublished).

36. P.P. Srivastava and G. Sudarshan, Phys. Kev. 110,765 (1958). 
37. J. Sandweiss, On the Spin of $\mathrm{K}$ Mesons from the Analysis of Antiproton Annihilations in Nuclear Emulsions (Thesis), UCRL-3577, October 31 , 1956 (unpublished).

38. I would like to thank Mr. Wonyong Lee for advice on the subject of this section and Mrs. Marjory Simmons for programming the calculations on the 650 and 704 IBM computers.

39. Protons with $\mathrm{T}_{\mathrm{p}} \leqslant 10 \mathrm{Mev}$ did not produce visible tracks in the bubble chamber and were not included in the evaluation of the proton multiplicity. However we included the protons that we estimate were recorded as $\pi^{+}$during the scanning $\left(0.24 \mathrm{p}^{+}\right.$per star).

40. A. G. Ekspong and B.E. Ronne; Nuovo cimento 10, 27 (1959).

41. G. Goldhaber, T. Kalogeropoulos, and R. Silberberg, Phys. Rev. 110. 1474 (1958).

42. N. Metropolis, R. Bivins, M. Storm, J.B. Miller, G. Friedlander, and A. Turkevich, Phys. Rev. 110, 204 (1958): also private communication from Dr. Metropolis.

43. I. Dostrovsky, P. Rabinowitz, and R. Bivins, Phys. Rev. 111, 1659 (1959).

44. M.G.K. Menon, H. Mụirhead, and O. Rochat, Phil. Mag. 41, 583 (1950).

45. G.E. Belovitskii, J. Exptl. Theoret. Phys. U.S.S.R. 35, 838 (1958); translation: Soviet Phys. JETP 8, 581 (1959).

46. R. M. Frank, J. L. Gammel, and K. M. Watson, Phys. Rev. 101, 891 (1956).

47. K.C. Wang, T. - T. Wang, D. T. Ding, L. N. Dubrovskii, E. N. Kladnitskaia, and M.I. Solov'ev; J. Exptl. Theoret. Phys. U.S.S.R. 35, 899 (1958); translation: Soviet Phys. JETP $\underline{8}, 625$ (1959).

48. A.E. Ignatenko, A.I. Mukhin, E.B. Ozerov, and B. M. Pontecorvo, J'. Expt1. Theoret. Phys. U.S.S.R. 31, 546 (1956); translation: Soviet Phys. JETP $\underline{4}, 351$ (1957).

49. M. Blau and A.R. Oliver, Phys. Rev. 102, 489 (1956).

50. V.P. Dzelepov, V.G. Ivanov, M.S. Kozodaev, V.T. Osipenkov, N.I. Petrov and V.A. Rusakov, J. Exptl. Theoret. Phys. U.S.S.R. 31, 923 (1956); translation: Soviet Phys. JETP.' 4 , 864 (1957).

51. J.O. Kessler and L.M. Lederman, Phys. Rev. 94,689 (1954).

52. G. Bernardini and F. Levy, Phys. Rev. 84, 610 (1951).

53. M. Blau and M. Coulton, Phys. Rev. 96, 150 (1954). 
54. The author'is indebted to Professors K. M. Watson and O. Chamberlain for discussions and advice on this problem.

55. A.H. Rosenfeld, Phys. Rev. 96, 139 (1959).

56. Iu. M, Kazarinov and Iu. N. Simonov, J. Exptl. Theoret. Phys. U.S.S.R. 38, 78 (1958); translation: Soviet.Phys. JETP 8, 56 (1959).

57. Iu. D. Prokoshkin and A. A. Tiapkin, J. Exptl. Theoret. Phys. U.S.S.R. 32, 750 (1957); translation: Soviet Phys. JETP 5, 618 (1957).

58 A.P. Batson. B. B. Culwick, H.P. Klepp, and L. Riddiford, Proc. Roy. Soc. (London) A, 251, 233 (1959). 
This report was prepared as an account of Government sponsored work. Neither the United States, nor the Commission, nor any person acting on behalf of the Commission:

A. Makes any warranty or representation, expressed or implied, with respect to the accuracy, completeness, or usefulness of the information contained in this report, or that the use of any information, apparatus, method, or process disclosed in this report may not infringe privately owned rights; or

B. Assumes any liabilities with respect to the use of, or for damageo regulting from the lise of any information, apparatus, method, or process disclosed in this report.

As used in the above, "person acting on behalf of the Commission" includes any employee or contractor of the Commission, or employee of such contractor, to the extent that such employee or contractor of the Commission, or employee of such contractor prepares, disseminates, or provides access to, any information pursuant to his employment or contract with the Commission, or his employment with such contractor. 